\title{
QUIVER VARIETIES AND SYMMETRIC PAIRS
}

\author{
YIQIANG LI
}

\begin{abstract}
We study fixed-point loci of Nakajima varieties under symplectomorphisms and their antisymplectic cousins, which are compositions of a diagram isomorphism, a reflection functor, and a transpose defined by certain bilinear forms. These subvarieties provide a natural home for geometric representation theory of symmetric pairs. In particular, the cohomology of a Steinberg-type variety of the symplectic fixed-point subvarieties is conjecturally related to the universal enveloping algebra of the subalgebra in a symmetric pair. The latter symplectic subvarieties are further used to geometrically construct an action of a twisted Yangian on a torus equivariant cohomology of Nakajima varieties. In the type $A$ case, these subvarieties provide a quiver model for partial Springer resolutions of nilpotent Slodowy slices of classical groups and associated symmetric spaces, which leads to a rectangular symmetry and a refinement of Kraft-Procesi row/column removal reductions.
\end{abstract}

\section{Contents}

1. Introduction

2. Nakajima varieties

2.1. Graph

2.2. The variety $\Lambda_{\zeta \mathbb{C}}(\mathbf{v}, \mathbf{w})$

2.3. Quiver varieties $\mathfrak{M}_{\zeta}(\mathbf{v}, \mathbf{w})$ and $\mathfrak{M}_{0}(\mathbf{v}, \mathbf{w})$

2.4. Stability condition

3. Isomorphisms on Nakajima varieties

3.1. Reflection functors

3.2. The isomorphism $\tau$

3.3. The diagram isomorphism $a$

4. Geometric properties of $\sigma$-quiver varieties

4.1. The $\sigma$-quiver varieties, I: $\zeta$ generic

4.2. The $\sigma$-quiver varieties, II: $\zeta=\left(0, \zeta_{\mathbb{C}}\right)$

4.3. Weyl group action on $\sigma$-quiver varieties

4.4. Symplectic structure on $\mathfrak{S}_{\zeta}(\mathbf{v}, \mathbf{w})$

5. Quiver varieties and symmetric pairs

5.1. Restriction diagram

5.2. A characterization of $\mathfrak{S}_{\zeta}(\mathbf{v}, \mathbf{w})^{\mathrm{T}}$

5.3. Coideal structure

Received by the editors January 15, 2018, and, in revised form, October 15, 2018, and November 2, 2018.

2010 Mathematics Subject Classification. Primary 16S30, 14J50, 14L35, 51N30, 53D05.

Key words and phrases. Nakajima variety, partial Springer resolution, nilpotent Slodowy slices of classical groups, rectangular symmetry, column/row removal reduction, symmetric pairs, $\mathcal{K}-$ matrix.

This work was partially supported by the National Science Foundation under the grant DMS 1801915 . 
5.4. The stable map Stabe 24

5.5. Universal $\mathcal{K}$-matrix 25

5.6. Twisted Yangian via the FRT formalism 27

6. Example I: Cotangent bundles of isotropic flag varieties 28

6.1. Notation 29

6.2. Identification with $\sigma$-quiver varieties

7. Nakajima-Maffei isomorphism and symmetry 32

7.1. Nakajima-Maffei theorem

7.2. Rectangular symmetry 34

7.3. Column/row-removal reductions 36

8. Example II: Partial resolutions of nilpotent Slodowy slices 37

8.1. Maffei's morphism and the bilinear forms on $\widetilde{V}$ and $W$

8.2. Maffei's morphism and reflection functors 40

8.3. $\sigma$-quiver varieties and partial resolutions of nilpotent Slodowy slices 42

8.4. Rectangular symmetry for classical groups 44

8.5. Column/row removal reductions for classical groups 45

9. Fixed-points and categorical quotients 46

9.1. Polynomial invariants on $\mathbf{M}\left(\mathbf{v}^{0}, \mathbf{w}^{0}\right)^{\tau} \quad 46$

9.2. The closed immersion $\iota$ 47

10. Quiver varieties and symmetric spaces

10.1. The antisymplectic automorphism $\hat{\tau}_{\zeta}$

10.2. The $\hat{\sigma}$-quiver varieties

10.3. $\hat{\sigma}$-quiver varieties of type A 51

Acknowledgments

References 53

\section{INTRODUCTION}

To a Dynkin diagram of ADE type, one can attach a simply laced complex simple Lie algebra, say $\mathfrak{g}$, and a class of Nakajima's quiver varieties [N94, N98]. The latter provides a natural home for a geometric representation theory of the former. If the algebra $\mathfrak{g}$ is further equipped with an involution, it yields a complex Cartan decomposition of $\mathfrak{g}$ :

$$
\mathfrak{g}=\mathfrak{k} \oplus \mathfrak{p},
$$

where $\mathfrak{k}$ is the fixed-point subalgebra under involution, and $\mathfrak{p}$ is the eigenspace of eigenvalue -1 . The pair $(\mathfrak{g}, \mathfrak{k})$ is a so-called symmetric pair and $\mathfrak{p}$ is the associated symmetric space. The purpose of this paper is to develop a geometric theory for the symmetric pair $(\mathfrak{g}, \mathfrak{k})$ and its symmetric space $\mathfrak{p}$ by using Nakajima varieties together with their fixed-point loci under certain symplectic and antisymplectic involutions.

Thanks to É. Cartan, the classification of symmetric pairs is equivalent to the classification of real simple Lie algebras, which is given by Satake diagrams [H, OV]. These are bicolor Dynkin diagrams with black or white vertices, equipped with diagram involutions. Representation theory of symmetric pairs was developed under the influence of Harish-Chandra's theory of $\left(\mathfrak{g}, K_{\mathbb{R}}\right)$-modules with $K_{\mathbb{R}}$ a real adjoint group of $\mathfrak{k}([\mathrm{D}])$. A quantum version was obtained later by Letzter in [Le], where a 
coideal subalgebra $\mathbf{U}_{q}^{\prime}(\mathfrak{k})$ of the quantum algebra $\mathbf{U}_{q}(\mathfrak{g})$ is used as a $q$-analogue of the universal enveloping algebra of $\mathfrak{k}$.

Recently, the algebra $\mathbf{U}_{q}^{\prime}(\mathfrak{k})$ of type AIII/AIV without black vertices found its applications in the study of orthosymplectic Lie superalgebras by Bao and Wang [BW13] and even special orthogonal Lie algebras by Ehrig and Stroppel [ES13, independently. A new canonical basis was constructed for certain tensor modules of $\mathbf{U}_{q}^{\prime}(\mathfrak{k})$ in BW13, for idempotented $\mathbf{U}_{q}^{\prime}(\mathfrak{k})$ in BKLW, LW15, and finally a general theory of canonical basis for $\mathfrak{k}$ of any type was obtained in [BW16]. These works have inspired many developments in various directions, such as categorification BSWW] and K-matrix BaK16.

In the work BKLW], there is a geometric realization of $\mathbf{U}_{q}^{\prime}(\mathfrak{k})$ of type AIII/AIV without black vertices by using $n$-step isotropic flag varieties, in the spirit of Beilinson, Lusztig, and MacPherson's influential work BLM. In light of the role of loc. cit. in the works G91 by Ginzburg and N94, N98 by Nakajima, the geometric favor in BKLW], as the tip of the iceberg, strongly suggests the existence of a new class of quiver varieties for a general $\mathfrak{k}$ parallel to Nakajima varieties for $\mathfrak{g}$. Such an existence is conjectured independently, and maybe earlier, by Wang through his iProgram in [BW13, Introduction]. This new class of quiver varieties, called $\sigma$-quiver varieties, turns out to be fixed-point subvarieties of Nakajima varieties under certain symplectic involutions. More precisely, the symplectic involution $\sigma$ is a composition of a diagram involution, a reflection functor, and a symplectic transpose induced from certain bilinear forms. Note that the prototype of the involution $\sigma$ has been used in [N03, Section 9] (see also [VV03, 4.6]) for reinterpreting Lusztig's opposition L00b, which serves as a crucial ingredient in a construction of canonical bases. As we learned from [N18, and via private communication, it is known to Nakajima that in an affine analogue of [N15, A(iv)], fixed-point subvarieties of $\sigma$ on the regular parts of Nakajima varieties provide a quiver model for SO/Sp-instantons moduli spaces on ALE spaces, similar to the instantons-moduli-space origin KN90. of Nakajima varieties; see also Remark 9.2.4(3).

Just like Nakajima varieties, type $A \sigma$-quiver varieties possess many desirable properties.

Theorem A (Theorems 6.2.1, 8.3.3. Corollary 8.3.4). Nilpotent Slodowy slices of $\mathfrak{k}$ of type AI/AII and their partial Springer resolutions are examples of type A $\sigma$ quiver varieties.

In type AI/AII, the algebra $\mathfrak{k}$ is an orthogonal/symplectic Lie algebra, and thus we recover the geometry used in [BKLW]. Theorem $\mathrm{A}$ is a classical analogue of the well-known Nakajima-Maffei theorem that Nakajima varieties of type A are nilpotent Slodowy slices of $\mathfrak{s l}_{n}$ and their partial Springer resolutions [N94, M05. There are two easy but interesting applications from Theorem A Note that the $\mathfrak{s l}_{n}$-version, presented in Sections 7.2 and 7.3, has been done by Henderson [H15]. The first one is a symmetry in classical groups.

Theorem B (Theorem 8.4.1, Remark 8.4.2). There is a rectangular symmetry for partial Springer resolutions of nilpotent Slodowy slices of classical groups, that is, if the partitions involved can be fit into a rectangle of a certain size (see Figure 2), then the associated varieties are isomorphic.

The rectangular symmetry is further applied to prove a conjecture in Henderson and Licata's work [HL14] on Springer resolutions of two-row nilpotent Slodowy 
slices of classical groups, and recover relevant results in loc. cit. and W15]; see Example 8.4.3.

The second one is an enhancement of Kraft-Procesi's column/row removal reductions which play critical roles in the study of minimal singularities in classical nilpotent orbits. Kraft-Procesi KP82 showed smooth equivalences of singularities between nilpotent Slodowy slices $S_{\mu^{\prime}, \lambda}^{\mathfrak{g}}$ and $S_{r e d\left(\mu^{\prime}\right), \operatorname{red}(\lambda)}^{\mathfrak{g}^{\prime}}$ for certain classical Lie algebras $\mathfrak{g}$ and $\mathfrak{g}^{\prime}$, where $\operatorname{red}\left(\mu^{\prime}\right)$, (resp., $\left.\operatorname{red}(\lambda)\right)$ is obtained from partition $\mu^{\prime}$ (resp., $\lambda$ ) by removing certain rows/columns from $\mu^{\prime}$ (resp., $\lambda$ ); see Figure 3 .

Theorem C (Propositions 8.5.1 8.5.2). The nilpotent Slodowy slices in KraftProcesi's column/row removal reductions in KP82] are isomorphic.

Nilpotent orbits and their intersections with Slodowy slices in $\mathfrak{k}$ have been studied via categorical quotients in [KP82, K90, N94, Remark 8.5 (4)], and [N15, Appen$\operatorname{dix} \mathrm{A}(\mathrm{i})-\mathrm{A}(\mathrm{iv})]$. The latter approach, which is quite restricted, is closely related to the approach we take in this paper. They represent two different orders of taking GIT quotients and taking fixed points. A closed immersion, which is conjecturally isomorphic, between varieties which appeared from these two approaches is established in Proposition 9.2.1.

In a parallel direction, nilpotent orbits and, more generally, nilpotent Slodowy slices in the symmetric space $\mathfrak{p}$ have been studied by Kostant and Rallis [KR71], Sekiguchi [S84, and Ohta [086]. They have important applications in the orbit method of real reductive groups [V86, V89]. A slight alteration of the transpose in the involution $\sigma$ yields an antisymplectic involution $\hat{\sigma}$. Its fixed-point subvariety, called a $\hat{\sigma}$-quiver variety, can be regarded as the quiver variety for the symmetric space $\mathfrak{p}$, since results similar to Theorems A C remain valid in this setting (see Section [10). To this end, the geometries surrounding nilpotent elements in the triple $(\mathfrak{g}, \mathfrak{k}, \mathfrak{p})$ in (1) have their quiver counterparts:

Nakajima varieties, symplectic subvarieties, Lagrangian subvarieties.

With Theorems $\mathrm{A} C \mathrm{C}$ in hand, it is expected that there is a geometric representation theory for $\mathfrak{k}$ via general $\sigma$-quiver varieties, parallel to Nakajima's original theory for $\mathfrak{g}$. A further study shows that $\sigma$-quiver varieties and their Lagrangian cousins admit many favorable properties inherited from ambient Nakajima varieties. In particular, they are nonsingular, if the ambient Nakajima variety is so, and they carry a Weyl group action. The new Weyl groups contains Weyl groups of type $\mathrm{B}_{\ell} / \mathrm{C}_{\ell} / \mathrm{F}_{4}$. A Weyl group action of type $\mathrm{G}_{2}$ is realized by using an automorphism of order 6 on Nakajima varieties. Furthermore, a conjecture is formulated in the following, with supporting evidence given in Proposition 5.3 .3 and (66).

Conjecture (Conjecture 5.3.4). Let $(\mathfrak{g}, \mathfrak{k})$ be a symmetric pair listed in Table 1 in Section 5.3. There is a nontrivial algebra homomorphism from the enveloping algebra of $\mathfrak{k}$ to the top Borel-Moore homology of the $\sigma$-fixed-point, for a certain $\sigma$, of a Steinberg-type variety in the setting of Nakajima varieties.

From the table, one observes that Conjecture 5.3.4 if it holds, would provide a new geometric construction of the universal enveloping algebra of simple Lie algebras of type $\mathrm{B}$ and their representations. In addition to developing a geometric/quiver theory of $\mathfrak{k}$ (and $\mathfrak{p}$ ), there is a substantial interest in making a connection 
with the original Nakajima theory for $\mathfrak{g}$ to have a more interesting theory for $(\mathfrak{g}, \mathfrak{k})$ modules. The following theorem reflects such a flavor and is obtained by applying the machinery of Maulik-Okounkov's R-matrix [MO12,N16] to $\sigma$-quiver varieties.

Theorem D (Theorem 5.6.2). There is a $\left(y(\mathfrak{g}), y_{\sigma}\right)$-action on the localized torus equivariant cohomology of Nakajima varieties, where $y(\mathfrak{g})$ is the Yangian of $\mathfrak{g}$ and $y_{\sigma}$ is a twisted Yangian constructed in this paper via a geometric $\mathcal{K}$-matrix.

The twisted Yangian $y_{\sigma}$ should coincide with its algebraic counterpart, which can be traced back to Cherednik's work Ch84; see [M07, GRWa, GRWb. It is our hope that Theorem $\mathrm{D}$ will serve as a small step towards a geometric theory of $\left(\mathfrak{g}, K_{\mathbb{R}}\right)$-modules, which in turn will shed light on that of unitary representations of the associated real simple group.

Finally, we caution the reader that in the main body of the paper the automorphisms $\sigma$ and $\hat{\sigma}$ do not have to be involutive and the underlying graph is not necessarily of type ADE.

\section{NAKAJIMA VARIETIES}

In this section, we recall Nakajima's quiver varieties from the works N94, N96, N98.

2.1. Graph. Let $\Gamma$ be a graph without loops, with $I$ and $H$ being the vertex and arrow set, respectively. For each arrow $h$, let $\mathbf{o}(h)$ and $\mathbf{i}(h)$ be its outgoing and incoming vertex so that we can depict $h$ as $\mathbf{o}(h) \stackrel{h}{\rightarrow} \mathbf{i}(h)$. There is an involution on the arrow $\operatorname{set}^{-}: H \rightarrow H, h \mapsto \bar{h}$ such that $\mathbf{o}(\bar{h})=\mathbf{i}(h)$ and $\mathbf{i}(\bar{h})=\mathbf{o}(h)$.

Let $\mathbf{C}=\left(c_{i j}\right)_{i, j \in I}$ be the Cartan matrix of the graph $\Gamma$ defined by

$$
c_{i j}=2 \delta_{i, j}-\#\{h \in H \mid \mathbf{o}(h)=i, \mathbf{i}(h)=j\} .
$$

For each $i \in I$, we define a bijection $s_{i}: \mathbb{Z}^{I} \rightarrow \mathbb{Z}^{I}$ by $s_{i}(\xi)=\xi^{\prime}$, where $\xi_{j}^{\prime}=\xi_{j}-c_{j i} \xi_{i}$, $\xi=\left(\xi_{j}\right)_{j \in I}, \xi^{\prime}=\left(\xi_{j}^{\prime}\right)_{j \in I} \in \mathbb{Z}^{I}$. Let $\mathcal{W}$ be the the subgroup of $\operatorname{Aut}\left(\mathbb{Z}^{I}\right)$ generated by $s_{i}$ for all $i \in I$. The group $\mathcal{W}$ is the Weyl group of $\Gamma$. It admits a presentation with generators $s_{i}$ for all $i \in I$ and the following defining relations:

$$
\begin{aligned}
s_{i}^{2} & =1 & & \forall i \in I . \\
s_{i} s_{j} & =s_{j} s_{i}, & & \text { if } c_{i j}=0 . \\
s_{i} s_{j} s_{i} & =s_{j} s_{i} s_{j}, & & \text { if } c_{i j}=-1 .
\end{aligned}
$$

For a fixed $\mathbf{w}=\left(\mathbf{w}_{i}\right)_{i \in I} \in \mathbb{Z}^{I}$, we define a second (affine) $\mathcal{W}$-action on $\mathbb{Z}^{I}$ by $s_{i} * \mathbf{v}=\mathbf{v}^{\prime}$, where $\mathbf{v}=\left(\mathbf{v}_{i}\right)_{i \in I}, \mathbf{v}^{\prime}=\left(\mathbf{v}_{i}^{\prime}\right)_{i \in I} \in \mathbb{Z}^{I}$ such that $\mathbf{v}_{i}^{\prime}=\mathbf{v}_{i}-\sum_{j \in I} c_{i j} \mathbf{v}_{j}+\mathbf{w}_{i}$ and $\mathbf{v}_{j}^{\prime}=\mathbf{v}_{j}$ if $j \neq i$. We will put a subscript $\mathbf{w}$ under $*$, that is, $s_{i} *_{\mathbf{w}} \mathbf{v}$, if needed. If $w=s_{i_{1}} s_{i_{2}} \cdots s_{i_{l}}$ is a sequence of simple reflections, we set $w * \mathbf{v}=s_{i_{1}} * \cdots * s_{i_{l}} * \mathbf{v}$. We have

$$
\mathbf{C}\left(s_{i} *_{\mathbf{w}} \mathbf{v}\right)=s_{i}(\mathbf{C v}-\mathbf{w})+\mathbf{w} .
$$

2.2. The variety $\Lambda_{\zeta_{\mathbb{C}}}(\mathbf{v}, \mathbf{w})$. Let $V=\bigoplus_{i \in I} V_{i}$ and $W=\bigoplus_{i \in I} W_{i}$ be two finitedimensional $I$-graded vector spaces over the complex field $\mathbb{C}$ of dimension vectors $\mathbf{v}=\left(\mathbf{v}_{i}\right)_{i \in I}$ and $\mathbf{w}=\left(\mathbf{w}_{i}\right)_{i \in I}$, respectively. We consider the vector space

$$
\mathbf{M}(\mathbf{v}, \mathbf{w}) \equiv \mathbf{M}(V, W)=\bigoplus_{h \in H} \operatorname{Hom}\left(V_{\mathbf{o}(h)}, V_{\mathbf{i}(h)}\right) \oplus \bigoplus_{i \in I} \operatorname{Hom}\left(W_{i}, V_{i}\right) \oplus \operatorname{Hom}\left(V_{i}, W_{i}\right) .
$$


A typical element in $\mathbf{M}(\mathbf{v}, \mathbf{w})$ will be denoted by $\mathbf{x} \equiv(x, p, q) \equiv\left(x_{h}, p_{i}, q_{i}\right)_{h \in H, i \in I}$, where $x_{h} \in \operatorname{Hom}\left(V_{\mathbf{o}(h)}, V_{\mathbf{i}(h)}\right), p_{i} \in \operatorname{Hom}\left(W_{i}, V_{i}\right)$, and $q_{i} \in \operatorname{Hom}\left(V_{i}, W_{i}\right)$.

Let

$$
\mathrm{G}_{\mathbf{v}} \equiv \mathrm{G}_{V}=\prod_{i \in I} \mathrm{GL}\left(V_{i}\right), \quad \mathrm{G}_{\mathbf{w}} \equiv \mathrm{G}_{W}=\prod_{i \in I} \mathrm{GL}\left(W_{i}\right) .
$$

The group $\mathrm{G}_{\mathbf{v}}$ acts from the left on $\mathbf{M}(\mathbf{v}, \mathbf{w})$ by conjugation. More precisely, for all $g=\left(g_{i}\right)_{i \in I} \in \mathbf{G}_{\mathbf{v}}$ and $\mathbf{x} \in \mathbf{M}(\mathbf{v}, \mathbf{w})$, we define $g \cdot \mathbf{x}=\mathbf{x}^{\prime} \equiv\left(x_{h}^{\prime}, p_{i}^{\prime}, q_{i}^{\prime}\right)$, where $x_{h}^{\prime}=g_{\mathbf{i}(h)} x_{h} g_{\mathbf{o}(h)}^{-1}, p_{i}^{\prime}=g_{i} p_{i}$ and $q_{i}^{\prime}=q_{i} g_{i}^{-1}$ for all $h \in H$ and $i \in I$. Similarly, let $\mathrm{G}_{\mathbf{w}}$ acts conjugately on $\mathbf{M}(\mathbf{v}, \mathbf{w})$ from the left, i.e., for any $\mathrm{f}=\left(\mathrm{f}_{i}\right)_{i \in I} \in \mathrm{G}_{\mathbf{w}}$ and $\mathbf{x} \in \mathbf{M}(\mathbf{v}, \mathbf{w})$, we define $\mathbf{f} . \mathbf{x}=\mathbf{x}^{\prime} \equiv\left(x_{h}^{\prime}, p_{i}^{\prime}, q_{i}^{\prime}\right)$, where $x_{h}^{\prime}=x_{h}, p_{i}^{\prime}=p_{i} \mathrm{f}_{i}^{-1}$ and $q_{i}^{\prime}=\mathrm{f}_{i} q_{i}$ for all $h \in H$ and $i \in I$. It is clear that the $\mathrm{G}_{\mathbf{v}}$-action and $\mathrm{G}_{\mathbf{w}}$-action commute.

The space $\mathbf{M}(\mathbf{v}, \mathbf{w})$ can be endowed with a symplectic structure, given by

$$
\omega\left(\mathbf{x}, \mathbf{x}^{\prime}\right)=\sum_{h \in H} \operatorname{tr}\left(\varepsilon(h) x_{h} x_{\bar{h}}^{\prime}\right)+\sum_{i \in I} \operatorname{tr}\left(p_{i} q_{i}^{\prime}-p_{i}^{\prime} q_{i}\right) \quad \forall \mathbf{x}, \mathbf{x}^{\prime} \in \mathbf{M}(\mathbf{v}, \mathbf{w}),
$$

where $\varepsilon: H \rightarrow\{ \pm 1\}$ is a fixed orientation function such that $\varepsilon(h)+\varepsilon(\bar{h})=0$ for all $h \in H$. The orientation of $H$ associated to $\varepsilon$ is $\Omega=\varepsilon^{-1}(1)$. Let

$$
\mu \equiv \mu_{\mathbb{C}}: \mathbf{M}(\mathbf{v}, \mathbf{w}) \rightarrow \bigoplus_{i \in I} \mathfrak{g l}\left(V_{i}\right)
$$

be the moment map associated to the $\mathrm{G}_{\mathbf{v}}$-action on the symplectic vector space $\mathbf{M}(\mathbf{v}, \mathbf{w})$. Its projection at the $i$ th component $\mathfrak{g l}\left(V_{i}\right)$ is given by

$$
\mu_{i}: \mathbf{M}(\mathbf{v}, \mathbf{w}) \rightarrow \mathfrak{g l}\left(V_{i}\right), \quad \mu_{i}(\mathbf{x})=\sum_{h \in H: \mathbf{i}(h)=i} \varepsilon(h) x_{h} x_{\bar{h}}-p_{i} q_{i} .
$$

Let $\zeta_{\mathbb{C}}=\left(\zeta_{\mathbb{C}}^{(i)}\right)_{i \in I} \in \mathbb{C}^{I}$. We regard $\zeta_{\mathbb{C}}$ as an element in $\bigoplus_{i \in I} \mathfrak{g l}\left(V_{i}\right)$ via the imbedding $\left(\zeta_{\mathbb{C}}^{(i)}\right)_{i \in I} \mapsto\left(\zeta_{\mathbb{C}}^{(i)} \operatorname{Id}_{V_{i}}\right)_{i \in I}$. Let

$$
\Lambda_{\zeta_{\mathbb{C}}}(\mathbf{v}, \mathbf{w}) \equiv \mu_{\mathbb{C}}^{-1}\left(\zeta_{\mathbb{C}}\right)=\left\{\mathbf{x} \in \mathbf{M}(\mathbf{v}, \mathbf{w}) \mid \mu_{i}(\mathbf{x})=\zeta_{\mathbb{C}}^{(i)} \quad \forall i \in I\right\}
$$

We shall use the notation $\Lambda_{\zeta_{\mathbb{C}}}(V, W)$ for $\Lambda_{\zeta_{\mathbb{C}}}(\mathbf{v}, \mathbf{w})$ if we want to emphasize the pair $(V, W)$. Note that $\Lambda_{\zeta_{\mathbb{C}}}(\mathbf{v}, \mathbf{w})$ is an affine algebraic variety. Note also that $\mu_{i}(g \cdot \mathbf{x})=g_{i} \mu_{i}(\mathbf{x}) g_{i}^{-1}=\zeta_{\mathbb{C}}^{(i)}$ for all $g \in \mathrm{G}_{\mathbf{v}}$ and $\mathbf{x} \in \Lambda_{\zeta_{\mathbb{C}}}(\mathbf{v}, \mathbf{w})$. So the $\mathrm{G}_{\mathbf{v}}$-action on $\mathbf{M}(\mathbf{v}, \mathbf{w})$ restricts to a $G_{\mathbf{v}}$-action on $\Lambda_{\zeta_{\mathbb{C}}}(\mathbf{v}, \mathbf{w})$. Similarly, for all $f \in G_{\mathbf{w}}$ and $\mathbf{x} \in \mathbf{M}(\mathbf{v}, \mathbf{w})$, we have $\mu_{i}($ f. $\mathbf{x})=\mu_{i}(\mathbf{x})$. Hence we have a $\mathrm{G}_{\mathbf{w}}$-action on $\Lambda_{\zeta_{\mathbb{C}}}(\mathbf{v}, \mathbf{w})$.

2.3. Quiver varieties $\mathfrak{M}_{\zeta}(\mathbf{v}, \mathbf{w})$ and $\mathfrak{M}_{0}(\mathbf{v}, \mathbf{w})$. Let $\xi=\left(\xi_{i}\right)_{i \in I} \in \mathbb{Z}^{I}$. We define a character $\chi \equiv \chi_{\xi}: \mathrm{G}_{\mathbf{v}} \rightarrow \mathbb{C}^{*}$ by

$$
\chi(g) \equiv \chi_{\xi}(g)=\prod_{i \in I} \operatorname{det}\left(g_{i}\right)^{-\xi_{i}} \quad \forall g \in \mathrm{G}_{\mathbf{v}} .
$$

Let $\mathbb{C}\left[\Lambda_{\zeta_{\mathbb{C}}}(\mathbf{v}, \mathbf{w})\right]^{\mathrm{G}_{\mathbf{v}}, \chi^{n}}$ be the space of regular functions $f$ on $\Lambda_{\zeta_{\mathbb{C}}}(\mathbf{v}, \mathbf{w})$ such that $f(g \cdot \mathbf{x})=\chi^{n}(g) f(\mathbf{x})$ for all $g \in \mathrm{G}_{\mathbf{v}}$ and $\mathbf{x} \in \Lambda_{\zeta_{\mathbb{C}}}(\mathbf{v}, \mathbf{w})$. Then the sum

$$
R_{\zeta}(\mathbf{v}, \mathbf{w})=\bigoplus_{n \in \mathbb{N}} \mathbb{C}\left[\Lambda_{\zeta_{\mathbb{C}}}(\mathbf{v}, \mathbf{w})\right]^{\mathrm{G}_{\mathbf{v}}, \chi^{n}}, \quad \zeta \equiv\left(\xi, \zeta_{\mathbb{C}}\right),
$$

becomes an $\mathbb{N}$-graded commutative algebra with a subalgebra

$$
R_{0}(\mathbf{v}, \mathbf{w})=\mathbb{C}\left[\Lambda_{\zeta_{\mathbb{C}}}(\mathbf{v}, \mathbf{w})\right]^{\mathrm{G}_{\mathbf{v}}, \chi^{0}}
$$


Following Nakajima [N94, N98, we define the quiver varieties: for any $\zeta \equiv\left(\xi, \zeta_{\mathbb{C}}\right) \in$ $\mathbb{Z}^{I} \times \mathbb{C}^{I}$

$$
\mathfrak{M}_{\zeta}(\mathbf{v}, \mathbf{w})=\operatorname{Proj} R_{\zeta}(\mathbf{v}, \mathbf{w}), \quad \mathfrak{M}_{0}(\mathbf{v}, \mathbf{w})=\operatorname{Spec} R_{0}(\mathbf{v}, \mathbf{w}) .
$$

The inclusion $R_{0}(\mathbf{v}, \mathbf{w}) \rightarrow R_{\zeta}(\mathbf{v}, \mathbf{w})$ of the two rings involved induces a projective morphism of algebraic varieties:

$$
\pi: \mathfrak{M}_{\zeta}(\mathbf{v}, \mathbf{w}) \rightarrow \mathfrak{M}_{0}(\mathbf{v}, \mathbf{w}) .
$$

The $G_{\mathbf{w}}$-action on $\Lambda_{\zeta \mathbb{C}}(\mathbf{v}, \mathbf{w})$, which commutes with the $G_{\mathbf{v}}$-action, induces $G_{\mathbf{w}^{-}}$ actions on $\mathfrak{M}_{\zeta}(\mathbf{v}, \mathbf{w})$ and $\mathfrak{M}_{0}(\mathbf{v}, \mathbf{w})$. It is clear that the proper map $\pi$ is $G_{\mathbf{w}^{-}}$ equivariant.

2.4. Stability condition. Fix an element $x=\left(x_{h}\right)_{h \in H}$ in the first component of $\mathbf{M}(\mathbf{v}, \mathbf{w})$ and an $I$-graded subspace $S=\left(S_{i}\right)_{i \in}$ of $V$; we say that $S$ is $x$-invariant if $x_{h}\left(S_{\mathbf{o}(h)}\right) \subseteq S_{\mathbf{i}(h)}$ for all $h \in H$. The standard dot product on $\mathbb{Z}^{I}$ is given by $a \cdot b=$ $\sum_{i \in I} a_{i} b_{i}$ for all $a, b \in \mathbb{Z}^{I}$. Following Nakajima, a point $\mathbf{x}=(x, p, q)$ in $\mathbf{M}(\mathbf{v}, \mathbf{w})$ is called $\xi$-semistable if the following two stability conditions are satisfied. Assume $S$ and $T$ are $I$-graded subspaces of $V$ of dimension vector $\mathbf{s}$ and $\mathbf{t}$, respectively. Then the stability conditions say that

$$
\text { If } S \text { is } x \text {-invariant and } S \subseteq \operatorname{ker} q \text {, then } \xi \cdot \mathbf{s} \leq 0 \text {. }
$$

If $T$ is $x$-invariant and $T \supseteq \operatorname{im} p$, then $\xi \cdot \mathbf{t} \leq \xi \cdot \mathbf{v}$.

Let $\Lambda_{\zeta_{\mathbb{C}}}^{\xi-s s}(\mathbf{v}, \mathbf{w})$ be the set of all $\xi$-semistable points in $\Lambda_{\zeta_{\mathbb{C}}}(\mathbf{v}, \mathbf{w})$. We see that $\Lambda_{\zeta \mathbb{C}}^{\xi-s s}(\mathbf{v}, \mathbf{w})$ is $\mathrm{G}_{\mathbf{v}}$-invariant. For convenience, let $[\mathbf{x}]$ denote the $\mathrm{G}_{\mathbf{v}}$-orbit of $\mathbf{x}$ in $\mathbf{M}(\mathbf{v}, \mathbf{w})$. From Mumford's geometric invariant theory, we have the following.

Proposition 2.4.1 ([N96, 3.ii]). The geometric points in $\mathfrak{M}_{\zeta}(\mathbf{v}, \mathbf{w})$ are $\Lambda_{\zeta_{\mathbb{C}}}^{\xi-s s}(\mathbf{v}, \mathbf{w}) /$ $\sim$, where the GIT equivalence relation $\sim$ is defined as $\mathbf{x} \sim \mathbf{y}$ if and only if $\overline{\mathbf{x}]} \cap \overline{[\mathbf{y}]} \cap$ $\Lambda_{\zeta_{\mathbb{C}}}^{\xi-s s}(\mathbf{v}, \mathbf{w}) \neq \varnothing$ where the overline denotes the Zariski closure of the underlying orbit in $\Lambda_{\zeta \mathbb{C}}(\mathbf{v}, \mathbf{w})$.

Recall that $\mathbf{C}$ is the Cartan matrix of the graph $\Gamma$. We set

$$
\begin{aligned}
R_{+} & =\left\{\gamma \in \mathbb{N}^{I} \mid{ }^{t} \gamma \mathbf{C} \gamma \leq 2\right\}-\{0\}, \\
R_{+}(\mathbf{v}) & =\left\{\gamma \in R_{+} \mid \gamma_{i} \leq \mathbf{v}_{i} \forall i \in I\right\}, \\
D_{\gamma} & =\left\{a \in \mathbb{C}^{I} \mid a \cdot \gamma=0\right\} .
\end{aligned}
$$

So the set $R_{+}$consists of positive roots of $\mathbf{C}$ and the set $D_{\gamma}$ is the wall defined by $\gamma$. Note that there is ${ }^{t} \gamma \mathbf{C} \gamma=2 \sum_{i \in I} \gamma_{i}^{2}-\sum_{h \in H} \gamma_{\mathbf{o}(h)} \gamma_{\mathbf{i}(h)}$.

Definition 2.4.2. A parameter $\zeta=(\xi, \zeta \mathbb{C}) \in \mathbb{Z}^{I} \times \mathbb{C}^{I}$ is called generic if it satisfies

$$
\xi \in \mathbb{Z}^{I} \backslash \bigcup_{\gamma \in R_{+}(\mathbf{v})} D_{\gamma} \quad \text { or } \quad \zeta_{\mathbb{C}} \in \mathbb{C}^{I} \backslash \bigcup_{\gamma \in R_{+}(\mathbf{v})} D_{\gamma} .
$$

Proposition 2.4.3 (N94, Theorem 2.8]). Assume that the parameter $\zeta$ is generic. Then the group $\mathrm{G}_{\mathbf{v}}$ acts freely on $\Lambda_{\zeta_{\mathbb{C}}}^{\xi-s s}(\mathbf{v}, \mathbf{w})$ and $\mathfrak{M}_{\zeta}(\mathbf{v}, \mathbf{w})=\Lambda_{\zeta_{\mathbb{C}}}^{\xi-s}(\mathbf{v}, \mathbf{w}) / \mathrm{G}_{\mathbf{v}}$, the GIT quotient of $\Lambda_{\zeta_{\mathbb{C}}}^{\xi-s s}(\mathbf{v}, \mathbf{w})$ by $\mathrm{G}_{\mathbf{v}}$. Moreover, $\mathfrak{M}_{\zeta}(\mathbf{v}, \mathbf{w})$ is smooth. 
Hence the geometric points of the quiver variety $\mathfrak{M}_{\zeta}(\mathbf{v}, \mathbf{w})$ under (9) are parametrized by the $\mathrm{G}_{\mathbf{v}}$-orbits in $\Lambda_{\zeta_{\mathbb{C}}}^{\xi \text {-ss }}(\mathbf{v}, \mathbf{w})$. We set

$$
\mathfrak{M}_{\zeta}(\mathbf{w})=\coprod_{\mathbf{v}} \mathfrak{M}_{\zeta}(\mathbf{v}, \mathbf{w}) .
$$

Assumption 2.4.4. The parameter $\zeta$ is assumed to be either generic or zero, unless otherwise stated.

Remark 2.4.5. Our $\xi$ is corresponding to the parameter $\zeta_{\mathbb{R}}$ where $\zeta_{\mathbb{R}}=\sqrt{-1} \xi$ in $\mathrm{N03}$.

\section{ISOMORPHISMS ON NAKAJIMA VARIETIES}

In this section, we introduce three classes of isomorphisms on Nakajima varieties. The fixed-point loci of their compositions, when they become automorphisms, will be studied in the next section.

3.1. Reflection functors. To each element $\omega \in \mathcal{W}$, Nakajima [N94, N03], Lusztig [L00, and Maffei [M02] define the so-called reflection functor

$$
S_{\omega}: \mathfrak{M}_{\zeta}(\mathbf{v}, \mathbf{w}) \rightarrow \mathfrak{M}_{\omega(\zeta)}(\omega * \mathbf{v}, \mathbf{w}) \quad \forall \zeta \text { subject to (9), }
$$

which is an isomorphism of varieties such that $S_{\omega^{\prime}} S_{\omega}=S_{\omega^{\prime} \omega}$. When $\omega$ is a simple reflection, the definition is very much like Bernstein, Gelfand, and Ponomarev's reflection functor $\mathrm{BGP}$, from which it is named.

Retain the pair of vector spaces $(V, W)$ of dimension vector $(\mathbf{v}, \mathbf{w})$. Fix $i \in I$ and set

$$
U_{i}=W_{i} \oplus \bigoplus_{h \in H: \mathbf{o}(h)=i} V_{\mathbf{i}(h)} .
$$

Let $V^{\prime}$ be a third vector space of dimension $\mathbf{v}^{\prime}=s_{i} * \mathbf{v}$ such that $V_{j}^{\prime}=V_{j}$ if $j \neq i$. In particular, $\operatorname{dim} V_{i}^{\prime}+\operatorname{dim} V_{i}=\operatorname{dim} U_{i}$. To a point $\mathbf{x} \in \mathbf{M}(\mathbf{v}, \mathbf{w})$, we set $a_{i}(\mathbf{x})=\left(q_{i}, x_{h}\right)_{h: \mathbf{o}(h)=i}$ and $b_{i}(\mathbf{x})=\left(p_{i}, \varepsilon(\bar{h}) x_{h}\right)_{h: \mathbf{i}(h)=i}$. Let $F$ be the pair of points $\left(\mathbf{x}, \mathbf{x}^{\prime}\right) \in \mathbf{M}(\mathbf{v}, \mathbf{w}) \times \mathbf{M}\left(\mathbf{v}^{\prime}, \mathbf{w}\right)$ such that the following conditions (R1) (R4) hold:

$$
\begin{aligned}
& 0 \longrightarrow V_{i}^{\prime} \stackrel{a_{i}\left(\mathbf{x}^{\prime}\right)}{\longrightarrow} U_{i} \stackrel{b_{i}(\mathbf{x})}{\longrightarrow} V_{i} \longrightarrow 0 \text { is exact, } \\
& a_{i}(\mathbf{x}) b_{i}(\mathbf{x})-a_{i}\left(\mathbf{x}^{\prime}\right) b_{i}\left(\mathbf{x}^{\prime}\right)=\zeta_{\mathbb{C}}^{\prime(i)}, \quad \zeta_{\mathbb{C}}^{\prime}=s_{i}\left(\zeta_{\mathbb{C}}\right), \\
& x_{h}=x_{h}^{\prime}, p_{j}=p_{j}^{\prime}, q_{j}=q_{j}^{\prime}, \quad \text { if } \mathbf{o}(h) \neq i, \mathbf{i}(h) \neq i, \text { and } j \neq i, \\
& \mu_{j}(\mathbf{x})=\zeta_{\mathbb{C}}^{(j)}, \mu_{j}\left(\mathbf{x}^{\prime}\right)=\zeta_{\mathbb{C}}^{\prime(j)}, \quad \text { if } j \neq i .
\end{aligned}
$$

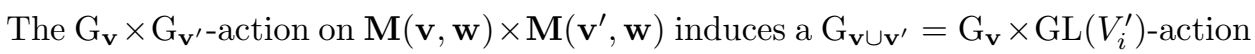
on $F$.

Assume that the parameter $\xi$ satisfies $\xi_{i}<0$ or $\zeta_{\mathbb{C}}^{(i)} \neq 0$. We have the following diagram:

$$
\Lambda_{\zeta_{\mathbb{C}}}^{\xi-s s}(V, W) \stackrel{\pi_{1}}{\longleftarrow} F^{s s}\left(V, V^{\prime}, W\right) \stackrel{\pi_{2}}{\longrightarrow} \Lambda_{s_{i}\left(\zeta_{\mathbb{C}}\right)}^{s_{i}(\xi)-s s}\left(V^{\prime}, W\right),
$$

where $F^{s s}\left(V, V^{\prime}, W\right)=F \cap\left(\Lambda_{\zeta_{\mathbb{C}}}^{\xi-s s}(V, W) \times \Lambda_{s_{i}\left(\zeta_{\mathbb{C}}\right)}^{s_{i}(\xi)-s s}\left(V^{\prime}, W\right)\right), \pi_{1}$, and $\pi_{2}$ are the natural projections. It is known that $\pi_{1}$ and $\pi_{2}$ are $\operatorname{GL}\left(V_{i}^{\prime}\right)$ and $\operatorname{GL}\left(V_{i}\right)$ principal bundles, respectively. This induces isomorphisms of varieties:

$$
\mathfrak{M}_{\zeta}(\mathbf{v}, \mathbf{w}) \stackrel{\pi_{1}}{\longleftarrow} \mathrm{G}_{\mathbf{v} \cup \mathbf{v}^{\prime}} \backslash F^{s s}\left(V, V^{\prime}, W\right) \stackrel{\pi_{2}}{\longrightarrow} \mathfrak{M}_{s_{i}(\zeta)}\left(s_{i} * \mathbf{v}, \mathbf{w}\right) .
$$


The simple reflection $S_{i}$ on quiver varieties is defined by

$$
S_{i}=\pi_{2} \pi_{1}^{-1}: \mathfrak{M}_{\zeta}(\mathbf{v}, \mathbf{w}) \rightarrow \mathfrak{M}_{s_{i}(\zeta)}\left(s_{i} * \mathbf{v}, \mathbf{w}\right), \quad \text { if } \xi_{i}<0 \text { or } \zeta_{\mathbb{C}}^{(i)} \neq 0 .
$$

Since $\left(s_{i}(\xi)\right)_{i}>0$ if $\xi_{i}<0$, we can define the reflection $S_{i}$ when $\xi_{i}>0$, by switching the roles of $\mathbf{x}$ and $\mathbf{x}^{\prime}$. So if $\omega=s_{i_{1}} s_{i_{2}} \cdots s_{i_{l}} \in \mathcal{W}$ and $\zeta$ satisfies the condition (9), the reflection functor $S_{\omega}$ in (11) is defined to be

$$
S_{\omega}=S_{i_{1}} S_{i_{2}} \cdots S_{i_{l}}: \mathfrak{M}_{\zeta}(\mathbf{v}, \mathbf{w}) \rightarrow \mathfrak{M}_{\omega(\zeta)}(\omega * \mathbf{v}, \mathbf{w}) .
$$

When $\zeta=0$, the reflection functor $S_{\omega}: \mathfrak{M}_{0}(\mathbf{v}, \mathbf{w}) \rightarrow \mathfrak{M}_{0}(\omega * \mathbf{v}, \mathbf{w})$ is defined to be the identity morphism when $\omega * \mathbf{v}=\mathbf{v}$, following [L00, 2.1].

If we let $\mathrm{G}_{\mathbf{w}}$ act diagonally on $\mathbf{M}(\mathbf{v}, \mathbf{w}) \times \mathbf{M}\left(\mathbf{v}^{\prime}, \mathbf{w}\right)$ in the above construction, we see that the simple reflections $S_{i}$ and hence the general Weyl group action $S_{\omega}$ are $\mathrm{G}_{\mathbf{w}}$-equivariant.

3.2. The isomorphism $\tau$. A finite-dimensional vector space $E$ equipped with a nondegenerate bilinear form $(-,-)_{E}$ is called a formed space. To any linear transformation $T: E \rightarrow E^{\prime}$ between two formed spaces, we define its right adjoint $T^{*}: E^{\prime} \rightarrow E$ by the rule

$$
\left(T(e), e^{\prime}\right)_{E^{\prime}}=\left(e, T^{*}\left(e^{\prime}\right)\right)_{E} \quad \forall e \in E, e^{\prime} \in E^{\prime} .
$$

It is clear that the map $T \mapsto T^{*}$ defines an isomorphism $\operatorname{Hom}\left(E, E^{\prime}\right) \cong \operatorname{Hom}\left(E^{\prime}, E\right)$ of vector spaces. If further $E^{\prime \prime}$ is a formed space and $T^{\prime}: E^{\prime} \rightarrow E^{\prime \prime}$ is a linear transformation, then $\left(T^{\prime} T\right)^{*}=T^{*} T^{*}$.

Similarly, we can define the left adjoint $T^{!}$of $T$ by $\left(e^{\prime}, T(e)\right)_{E^{\prime}}=\left(T^{!}\left(e^{\prime}\right), e\right)_{E}$ for all $e \in E$ and $e^{\prime} \in E^{\prime}$. We have $\left(T^{*}\right)^{!}=T$ and $\left(T^{!}\right)^{*}=T$.

Let $\delta$ be either +1 or -1 . A formed space $E$ is called a $\delta$-formed space if the associated form $(-,-)_{E}$ on $E$ satisfies that $\left(e_{1}, e_{2}\right)_{E}=\delta\left(e_{2}, e_{1}\right)_{E}$ for all $e_{1}, e_{2} \in E$. When $\delta=1$, we have a symmetric form, while when $\delta=-1$, we have a symplectic form. In this case, the form $(-,-)_{E}$ is called a $\delta$-form. If $E^{\prime}$ is a $\delta^{\prime}$-formed space for some $\delta^{\prime} \in\{ \pm 1\}$, then we have $\left(T^{*}\right)^{*}=\delta \delta^{\prime} T$.

Assume the vector space $E$ is a formed space and admits an $I$-grading $E=$ $\bigoplus_{i \in I} E_{i}$. We call $E$ an I-graded formed space if the restriction $(-,-)_{E_{i}}$ of the form $(-,-)_{E}$ to each subspace $E_{i}$ is a nondegenerate form and $\left(E_{i}, E_{j}\right)_{E}=0$ if $i \neq j$. Let $E$ be an $I$-graded formed space and fix a function $\underline{\delta}=\left(\delta_{i}\right)_{i \in I} \in\{ \pm 1\}^{I}$. We call $E$ a $\underline{\delta}$-formed space, or a formed space with sign $\underline{\delta}$, if the restriction $(-,-)_{E_{i}}$ is a $\delta_{i}$-form for all $i \in I$. We call $\underline{\delta}$ the sign of $E$.

Recall the pair $(V, W)$ of vector spaces of dimension vector $(\mathbf{v}, \mathbf{w})$ and $\mathbf{M}(\mathbf{v}, \mathbf{w})$ from Section 2.2. Assume that $V$ and $W$ are two $I$-graded formed spaces. We define an automorphism

$$
\tau: \mathbf{M}(\mathbf{v}, \mathbf{w}) \rightarrow \mathbf{M}(\mathbf{v}, \mathbf{w}), \quad \mathbf{x}=\left(x_{h}, p_{i}, q_{i}\right) \mapsto{ }^{\tau} \mathbf{x}=\left({ }^{\tau} x_{h},{ }^{\tau} p_{i},{ }^{\tau} q_{i}\right),
$$

where ${ }^{\tau} x_{h}=\varepsilon(h) x_{\bar{h}}^{*},{ }^{\tau} p_{i}=-q_{i}^{*}$ and ${ }^{\tau} q_{i}=p_{i}^{*}$ for all $h \in H$ and $i \in I$. Its inverse is defined by taking the left adjoints, that is, $\tau^{-1}(\mathbf{x})=\left({ }^{\tau^{-1}} x_{h},{ }^{\tau^{-1}} p_{i},{ }^{\tau^{-1}} q_{i}\right)$, where $\tau^{-1} x_{h}=\varepsilon(\bar{h}) x_{\bar{h}}^{!}, \tau^{-1} p_{i}=q_{i}^{!}$, and ${ }^{\tau^{-1}} q_{i}=-p_{i}^{!}$.

By the properties of taking adjoints, we have $\mu_{i}\left({ }^{\tau} \mathbf{x}\right)=-\mu_{i}(\mathbf{x})^{*}$. So the automorphism on $\mathbf{M}(\mathbf{v}, \mathbf{w})$ restricts to an isomorphism still denoted by $\tau$ :

$$
\tau: \Lambda_{\zeta_{\mathbb{C}}}(\mathbf{v}, \mathbf{w}) \rightarrow \Lambda_{-\zeta_{\mathbb{C}}}(\mathbf{v}, \mathbf{w})
$$




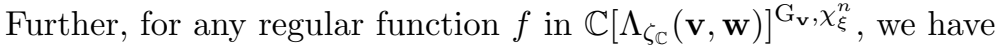

$$
\begin{array}{rlrl}
f \tau(g \cdot \mathbf{x}) & =f\left({ }^{\tau} g \cdot{ }^{\tau} \mathbf{x}\right) & & { }^{\tau} g_{i}=\left(g_{i}^{-1}\right)^{*} \\
& =\chi_{\xi}^{n}\left({ }^{\tau} g\right) f\left({ }^{\tau} \mathbf{x}\right) & & \\
& =\chi_{-\xi}^{n}(g) f \tau(\mathbf{x}) & \forall g \in \mathrm{G}_{\mathbf{v}}, \mathbf{x} \in \Lambda_{-\zeta_{\mathbb{C}}}(\mathbf{v}, \mathbf{w}) .
\end{array}
$$

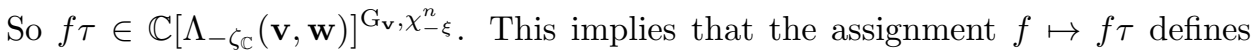
an isomorphism of graded associative algebras: $R_{\zeta}(\mathbf{v}, \mathbf{w}) \rightarrow R_{-\zeta}(\mathbf{v}, \mathbf{w})$, where $R_{\zeta}(\mathbf{v}, \mathbf{w})$ is from Section 2.3 .

The above isomorphism shows that the isomorphism on $\Lambda_{\zeta_{\mathbb{C}}}(\mathbf{v}, \mathbf{w})$ restricts to an isomorphism $\Lambda_{\zeta_{\mathbb{C}}}^{\xi-s s}(\mathbf{v}, \mathbf{w}) \rightarrow \Lambda_{-\zeta_{\mathbb{C}}}^{(-\xi)-s s}(\mathbf{v}, \mathbf{w})$. Due to ${ }^{\tau}(g \cdot \mathbf{x})={ }^{\tau} g \cdot{ }^{\tau} \mathbf{x}$, it further induces the isomorphism: recall that $[\mathbf{x}]$ denotes the $\mathrm{G}_{\mathbf{v}}$-orbit of $\mathbf{x}$,

$$
\tau_{\zeta}: \mathfrak{M}_{\zeta}(\mathbf{v}, \mathbf{w}) \rightarrow \mathfrak{M}_{-\zeta}(\mathbf{v}, \mathbf{w}), \quad[\mathbf{x}] \mapsto\left[{ }^{\tau} \mathbf{x}\right],
$$

such that the following diagram commutes:

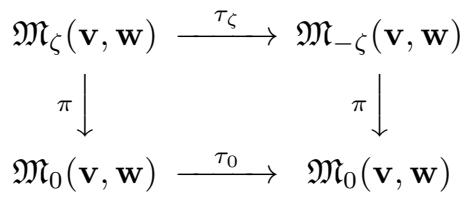

Now we show that the isomorphism $\tau_{\zeta}$ depends only on the forms on $W$.

Proposition 3.2.1. The $\tau_{\zeta}$ in (17) is independent of the choices of forms on $V$.

Proof. If we fix a basis for each vector space $V_{i}$ and $W_{i}$, then to give a form on $V_{i}$ or $W_{i}$ is the same as to give a certain invertible matrix, say $M_{i}$ or $N_{i}$. In this way, the right adjoints are presented as $x_{h}^{*}=M_{\mathbf{o}(h)}^{-1}{ }^{t} x_{h} M_{\mathbf{i}(h)}$ and $p_{i}^{*}=N_{i}^{-1 t} p_{i} M_{i}$ and $q_{i}^{*}=M_{i}^{-1 t} q_{i} N_{i}$ for all $h \in H$ and $i \in I$. If we attach to each $V_{i}$ a new form with associated matrix $\tilde{M}_{i}$, we can have a new automorphism, say $\tilde{\tau}$, on $\mathbf{M}(\mathbf{v}, \mathbf{w})$, and a new point ${ }^{\tilde{\tau}} \mathbf{x}$ for each $\mathbf{x} \in \mathbf{M}(\mathbf{v}, \mathbf{w})$. Set $g=\left(g_{i}\right)_{i \in I} \in \mathrm{G}_{\mathbf{v}}$ with $g_{i}=\tilde{M}_{i}^{-1} M_{i}$ $\forall i \in I$. Then the proposition follows from $g \cdot{ }^{\tau} \mathbf{x}=\tilde{\tau}^{\tilde{x}} \mathbf{x}$.

For $\mathrm{f} \in \mathrm{G}_{\mathbf{w}}$, we set ${ }^{\tau} \mathrm{f}=\left(\mathrm{f}^{-1}\right)^{*}$. Then $\tau(\mathrm{f} .[\mathbf{x}])={ }^{\tau} \mathrm{f} . \tau([\mathbf{x}])$ for all $\mathrm{f} \in \mathrm{G}_{\mathbf{w}}$ and $[\mathbf{x}] \in \mathfrak{M}_{\zeta}(\mathbf{v}, \mathbf{w})$.

Proposition 3.2.2. If $W$ is a formed space with sign $\delta_{\mathbf{w}}$, then the isomorphism $\tau_{\zeta}$ on $\mathfrak{M}_{\zeta}(\mathbf{v}, \mathbf{w})$ satisfies $\tau_{\zeta}^{4}=1$. Moreover, if the $\delta_{\mathbf{w}}$ is $\Gamma$-alternating, i.e., $\delta_{\mathbf{w}, \mathbf{o}(h)} \delta_{\mathbf{w}, \mathbf{i}(h)}$ $=-1$ for all $h \in H$, then $\tau_{\zeta}^{2}=1$.

Proof. From the property of taking adjoints twice with respect to $\delta$-forms, it is straightforward to see the first statement in the proposition. By Proposition 3.2.1. we can attach to each $V_{i}$ a symmetric form. Then for $\mathbf{x}=\left(x_{h}, p_{i}, q_{i}\right)$, we have $\tau^{2}(\mathbf{x})=\left(-x_{h},-\delta_{\mathbf{w}, i} p_{i},-\delta_{\mathbf{w}, i} q_{i}\right)$. Let $g=\left(-\delta_{\mathbf{w}, i} \mathrm{id}_{V_{i}}\right)_{i \in I}$; then we have $g \cdot \tau^{2}(\mathbf{x})=\mathbf{x}$. This implies that $\tau_{\zeta}^{2}=1$.

We now show that the isomorphism $\tau_{\zeta}$ commutes with the reflection functors. Recall the setting from Section 3.1. We fix a vertex $i \in I$ and a triple $\left(V, V^{\prime}, W\right)$ of $I$-graded vector spaces of dimension vector $\left(\mathbf{v}, \mathbf{v}^{\prime}, \mathbf{w}\right)$ such that $V_{j}=V_{j}^{\prime}$ for all $j \neq i$ and $\mathbf{v}^{\prime}=s_{i} * \mathbf{v}$. We assume that all spaces in this triple are $I$-graded formed spaces. For simplicity, let $B_{V}=(-,-)_{V}, B_{W}=(-,-)_{W}$ stand for the bilinear 
forms on $V$ and $W$, respectively. Similar to the isomorphism $\tau_{\zeta} \equiv \tau_{\zeta}\left(B_{V}, B_{W}\right)$ as above, we have an isomorphism

$$
\tau_{s_{i}(\zeta)} \equiv \tau_{s_{i}(\zeta)}\left(B_{V^{\prime}}, B_{W}\right): \mathfrak{M}_{s_{i}(\zeta)}\left(s_{i} * \mathbf{v}, \mathbf{w}\right) \rightarrow \mathfrak{M}_{-s_{i}(\zeta)}\left(s_{i} * \mathbf{v}, \mathbf{w}\right) .
$$

Lemma 3.2.3. We have $S_{i} \tau_{\zeta}\left(B_{V}, B_{W}\right)=\tau_{s_{i}(\zeta)}\left(B_{V^{\prime}}, B_{W}\right) S_{i}$, where $S_{i}$ is the reflection functor defined in Section 3.1 .

Proof. By Proposition 3.2.1 we can assume that the forms on $V_{j}$ and $V_{j}^{\prime}$ coincide for all $j \neq i$. We observe that $a_{i}\left({ }^{\tau} \mathbf{x}\right)=b_{i}(\mathbf{x})^{*}$ and $b_{i}\left({ }^{\tau} \mathbf{x}\right)=-a_{i}(\mathbf{x})^{*}$. So the short exact sequence in (R1) gives rise to the following short exact sequence:

$$
0 \longrightarrow V_{i} \stackrel{a_{i}\left({ }^{\tau} \mathbf{x}\right)}{\longrightarrow} U_{i} \stackrel{b_{i}\left({ }^{\tau} \mathbf{x}^{\prime}\right)}{\longrightarrow} V_{i}^{\prime} \longrightarrow 0 .
$$

Similarly, the equation in (2) yields the equality $a_{i}\left({ }^{\tau} \mathbf{x}^{\prime}\right) b_{i}\left({ }^{\tau} \mathbf{x}^{\prime}\right)-a_{i}\left({ }^{\tau} \mathbf{x}\right) b_{i}\left({ }^{\tau} \mathbf{x}\right)=$ $\zeta_{\mathbb{C}}^{\prime(i)}$. As a consequence, we have the commutative relation in the lemma.

3.3. The diagram isomorphism $a$. Let $a$ be an automorphism of $\Gamma$, that is, there are permutations of vertex and edge sets, both denoted by $a$, such that $a(\mathbf{o}(h))=$ $\mathbf{o}(a(h)), a(\mathbf{i}(h))=\mathbf{i}(a(h))$, and $a(\bar{h})=\overline{a(h)}$ for all $h \in H$. We further assume that $a$ is compatible with the function $\varepsilon$ in the definition of the moment map $\mu$ in Section 2.2. there exists a constant $c \equiv c_{a, \varepsilon} \in\{ \pm 1\}$ such that

$$
\varepsilon(a(h))=c \cdot \varepsilon(h) \forall h \in H .
$$

The automorphism $a$ on $\Gamma$ induces operations on $I$-graded vector spaces and vectors. If $V$ is an $I$-graded space, we denote $a(V)$ as the $I$-graded vector space whose $i$ th component is $V_{a^{-1}(i)}$. Similarly $a(\mathbf{v})$ is a vector whose $i$-entry is the $a^{-1}(i)$ th entry of $\mathbf{v}$. Given any point $\mathbf{x}=(x, p, q) \in \mathbf{M}(\mathbf{v}, \mathbf{w}) \equiv \mathbf{M}(V, W)$, we define a point

$$
a(\mathbf{x})=(a(x), a(p), a(q)) \in \mathbf{M}(a(\mathbf{v}), a(\mathbf{w})) \equiv \mathbf{M}(a(V), a(W))
$$

by

$$
a(p)_{i}=p_{a^{-1}(i)}, a(q)_{i}=q_{a^{-1}(i)}, a(x)_{h}=\varepsilon(h)^{\frac{1-c}{2}} x_{a^{-1}(h)} \quad \forall i \in I, h \in H .
$$

By definition, $\mu_{i}(a(\mathbf{x}))=\mu_{a^{-1}(i)}(\mathbf{x})$. Thus it induces a diagram isomorphism of finite order on Nakajima's varieties:

$$
a: \mathfrak{M}_{\zeta}(\mathbf{v}, \mathbf{w}) \rightarrow \mathfrak{M}_{a(\zeta)}(a(\mathbf{v}), a(\mathbf{w})) .
$$

The order of this isomorphism is the same as that of the diagram.

The isomorphism $a$ is a variant of diagram automorphisms studied in HL14. Just like loc. cit., it can be generalized as follows. Let us fix $\left(f^{0}, g^{0}\right) \in \mathrm{G}_{\mathbf{w}} \times \mathrm{G}_{\mathbf{v}}$; we can define an isomorphism $a_{\mathrm{f}^{0}, g^{0}}: \mathfrak{M}_{\zeta}(\mathbf{v}, \mathbf{w}) \rightarrow \mathfrak{M}_{a(\zeta)}(a(\mathbf{v}), a(\mathbf{w}))$ to be the composition of $a$ with the action of $\left(\mathrm{f}^{0}, g^{0}\right)$. Specifically, for any $[\mathbf{x}] \in \mathfrak{M}_{\zeta}(\mathbf{v}, \mathbf{w})$, the element $a_{\mathrm{f}^{0}, g^{0}}([\mathbf{x}])$ is represented by $a_{\mathrm{f}^{0}, g^{0}}(\mathbf{x})=\left(a_{\mathrm{f}^{0}, g^{0}}(x), a_{\mathrm{f}^{0}, g^{0}}(p), a_{\mathrm{f}^{0}, g^{0}}(q)\right)$, where for all $i \in I, h \in H$,

$$
\begin{array}{r}
a_{\mathrm{f}^{0}, g^{0}}(p)_{i}=g_{a^{-1}(i)}^{0} p_{a^{-1}(i)}\left(\mathrm{f}_{a^{-1}(i)}^{0}\right)^{-1}, \\
a_{\mathrm{f}^{0}, g^{0}}(q)_{i}=\mathrm{f}_{a^{-1}(i)}^{0} q_{a^{-1}(i)}\left(g_{a^{-1}(i)}^{0}\right)^{-1}, \\
a_{\mathrm{f}^{0}, g^{0}}(x)_{h}=\varepsilon(h)^{\frac{1-c}{2}} g_{\mathbf{i}\left(a^{-1}(h)\right)^{0}}^{0} x_{a^{-1}(h)}\left(g_{\mathbf{o}\left(a^{-1}(h)\right)}^{0}\right)^{-1} .
\end{array}
$$

Similar to Proposition 3.2.1 the isomorphism $a_{\mathrm{f}^{0}, g^{0}}$ is independent of the choice of $g^{0}$. Hence it makes sense to denote this isomorphism by $a_{\mathrm{fo}}$, and the $a$ in (20) is $a_{1}$. 
There is a permutation, $\mathrm{f} \mapsto{ }^{a} \mathrm{f}$, on $\mathrm{G}_{\mathbf{w}}$ given by $\left({ }^{a} \mathrm{f}\right)_{i}=\mathrm{f}_{a^{-1}(i)}$ for all $i \in I$. It is clear that

$$
a_{\mathrm{fo}}(\mathrm{f} .[\mathbf{x}])={ }^{a} \mathrm{f}^{0} \cdot a_{\mathrm{f}}([\mathbf{x}]) \quad \forall[\mathbf{x}] \in \mathfrak{M}_{\zeta}(\mathbf{v}, \mathbf{w}), \mathrm{f} \in \mathrm{G}_{\mathbf{w}} .
$$

It is also clear that the isomorphism $a$ is compatible with the reflection functor $S_{i}$ :

$$
a_{\mathrm{f} o} \circ S_{i}=S_{a(i)} \circ a_{\mathrm{fo}} .
$$

Subsequently, $S_{w_{0}} \circ a_{\mathrm{f} 0}=a_{\mathrm{f}} \circ S_{w_{0}}$ when $\Gamma$ is Dynkin and $w_{0}$ is the longest Weyl group element since $a\left(w_{0}\right)=w_{0}$. The two isomorphisms $\tau_{\zeta}$ and $a_{\mathrm{f} o}$ are compatible as well. Precisely,

$$
\tau_{a \zeta}\left(a\left(B_{V}\right), a\left(B_{W}\right)\right) a_{\mathrm{f}^{0}}=a_{\tau_{\mathrm{f}} \mathrm{o}} \tau_{\zeta}\left(B_{V}, B_{W}\right) .
$$

Finally, we remark that the composition $a \tau_{\zeta}$ is similar to automorphisms in E09] and a special case of $\tau_{\zeta}$ appeared in [KP82]; see additionally Section 9 .

\section{Geometric Properties of $\sigma$-Quiver VARIETIES}

In this section, we study the fixed-point subvarieties, called $\sigma$-quiver varieties, of the compositions of the three classes of isomorphisms of Nakajima varieties introduced in the previous section.

4.1. The $\sigma$-quiver varieties, I: $\zeta$ generic. In this subsection, we assume that $\zeta$ is generic. We consider the following isomorphism on quiver varieties:

$$
\sigma:=a S_{\omega} \tau_{\zeta}: \mathfrak{M}_{\zeta}(\mathbf{v}, \mathbf{w}) \rightarrow \mathfrak{M}_{-a \omega(\zeta)}(a(\omega * \mathbf{v}), a(\mathbf{w})),
$$

where $\tau_{\zeta}, S_{\omega}$, and $a$ are defined in (17), (11), and (20), respectively. We shall write $\sigma_{\zeta, \omega, a}$ for $\sigma$ if we want to emphasize that $\sigma$ depends on $\zeta, \omega$, and $a$. By the commutativity of the three isomorphisms from Lemma 3.2.3, (22), and (23), we have the following proposition.

Proposition 4.1.1. If the forms involved are $\delta$-forms and $\omega$ is of finite order, then the order of $\sigma$ is finite and a divisor of the least common multiple l.c.m. $\{4,|\omega|,|a|\}$.

By summing over all $\mathbf{v}$, we have an isomorphism.

$$
\sigma: \mathfrak{M}_{\zeta}(\mathbf{w}) \rightarrow \mathfrak{M}_{-a \omega(\zeta)}(a(\mathbf{w}))
$$

If $-a \omega(\zeta)=\zeta$ and $a(\mathbf{w})=\mathbf{w}$, then $\sigma$ becomes an automorphism on $\mathfrak{M}_{\zeta}(\mathbf{w})$. We set

$$
\mathfrak{S}_{\zeta}(\mathbf{w}) \equiv \mathfrak{M}_{\zeta}(\mathbf{w})^{\sigma}
$$

to be its fixed-point subvariety. If further $a(\boldsymbol{\omega} * \mathbf{v})=\mathbf{v}$, let

$$
\mathfrak{S}_{\zeta}(\mathbf{v}, \mathbf{w}) \equiv \mathfrak{M}_{\zeta}(\mathbf{v}, \mathbf{w})^{\sigma}
$$

be the fixed-point subvariety of $\mathfrak{M}_{\zeta}(\mathbf{v}, \mathbf{w})$ under the automorphism $\sigma$. Then we have

$$
\mathfrak{S}_{\zeta}(\mathbf{w})=\bigsqcup_{a(\boldsymbol{\omega} * \mathbf{v})=\mathbf{v}} \mathfrak{S}_{\zeta}(\mathbf{v}, \mathbf{w}), \quad \text { if }-a \boldsymbol{\omega}(\zeta)=\zeta, a(\mathbf{w})=\mathbf{w} .
$$

Definition 4.1.2. The varieties $\mathfrak{S}_{\zeta}(\mathbf{v}, \mathbf{w})$ and $\mathfrak{S}_{\zeta}(\mathbf{w})$ are called the $\sigma$-quiver varieties.

Before we proceed, we make a remark. 
Remark 4.1.3. A more general isomorphism $\sigma_{\mathrm{f}}$ can be defined by using $a_{\mathrm{f}}$, a generalization of $a$, in Section 3.3. To control its order in this case, $\mathrm{f}^{0}$ has to satisfy a compatibility assumption in HL14. Specifically, we can identify $W_{i}$ with $W_{a(i)}$ for all $i \in I$ due to $\mathbf{w}_{i}=\mathbf{w}_{a(i)}$. For each $i \in I$, let $m_{i}=\#\left\{a^{n}(i) \mid n \in \mathbb{Z}\right\}$. Fix $m$ such that $m_{i} \mid m \forall i \in I$. The compatibility condition for $\mathrm{f}^{0}$ reads $\mathrm{f}_{i}^{0} \mathrm{f}_{a(i)}^{0} \cdots \mathrm{f}_{a^{m-1}(i)}^{0}=1$ $\forall i \in I$. Then the order of $a_{\mathrm{f}}$ is $m$. For the sake of simplicity, we focus on the simpler version $\sigma$ instead of $\sigma_{\mathrm{f}}$.

The definition of $\sigma$-quiver varieties depends on the forms on $V$ and $W$. But by Proposition 3.2.1, it only depends on the form on $W$, which is recorded as follows.

Proposition 4.1.4. The variety $\mathfrak{S}_{\zeta}(\mathbf{v}, \mathbf{w})$ is independent of the choice of the form on $V$.

By combining Proposition 4.1.1 and Proposition 4.1.4 it yields the following.

Proposition 4.1.5. If $W$ is a $\delta_{\mathbf{w}}$-formed space and $\omega$ is of finite order, then the order of $\sigma$ is a divisor of l.c.m. $\{4,|\omega|,|a|\}$. If further the sign $\delta_{\mathbf{w}}$ is $\Gamma$-alternating and $a^{2}=\omega^{2}=1$, then $\sigma^{2}=1$.

The following example shows that $\sigma$-quiver varieties include quiver varieties.

Example 4.1.6. Let $\hat{\Gamma}$ be the product of four copies of $\Gamma$. Let $a$ be the obvious cyclic permutation of order 4 on $\hat{\Gamma}$. Then there is an automorphism $\sigma$ with $\omega=1$ on $\mathfrak{M}_{\hat{\Gamma}}=\mathfrak{M}_{\zeta}(\mathbf{v}, \mathbf{w}) \times \mathfrak{M}_{-\zeta}(\mathbf{v}, \mathbf{w}) \times \mathfrak{M}_{\zeta}(\mathbf{v}, \mathbf{w}) \times \mathfrak{M}_{-\zeta}(\mathbf{v}, \mathbf{w})$. If the space $W$ is a formed space of sign $\delta_{\mathbf{w}}$, then we see that $\mathfrak{S}_{\hat{\Gamma}} \cong \mathfrak{M}_{\zeta}(\mathbf{v}, \mathbf{w})$. In particular, if the $\delta_{\mathbf{w}}$ is alternating, then we only need two copies of $\Gamma$ to realize $\mathfrak{M}_{\zeta}(\mathbf{v}, \mathbf{w})$ as a $\sigma$-quiver variety.

It is well known, e.g., [I72, Proposition 1.3], E92, Proposition 3.4], or [CG, Lemma 5.11.1], that the fixed-point subvariety of an action of a reductive group, in particular, a finite group, on a smooth variety is smooth. If the automorphism $\sigma$ has a finite order $N$, then it is the same as a $\mathbb{Z}_{N}$-action on quiver varieties. So it gives rise to the following proposition.

Proposition 4.1.7. Assume that $\zeta$ is generic. The $\sigma$-quiver variety $\mathfrak{S}_{\zeta}(\mathbf{v}, \mathbf{w})$ is smooth, provided that it is nonempty and the order of $\sigma$ is finite.

The reflection functor $S_{\omega}$ does not always exist on $\mathfrak{M}_{0}(\mathbf{v}, \mathbf{w})$ and, if it exists, they are not isomorphic in general. So to define $\sigma$-quiver varieties as a fixedpoint locus on $\mathfrak{M}_{0}(\mathbf{v}, \mathbf{w})$ does not work in general. When the graph is Dynkin, the reflection functor does exist on the global/limit version $\mathfrak{M}_{0}(\mathbf{w})$ of $\mathfrak{M}_{0}(\mathbf{v}, \mathbf{w})$, thanks to Lusztig's work [L00, so in this case it is possible to define $\sigma$-quiver variety in $\mathfrak{M}_{0}(\mathbf{w})$ as a fixed-point locus, which is treated in the following section. The $\sigma$ quiver variety in $\mathfrak{M}_{0}(\mathbf{v}, \mathbf{w})$ is then obtained by taking the intersection of $\mathfrak{M}_{0}(\mathbf{v}, \mathbf{w})$ with the $\sigma$-quiver variety in $\mathfrak{M}_{0}(\mathbf{w})$. Here, instead, we define the following:

$$
\begin{aligned}
\mathfrak{S}_{1}(\mathbf{v}, \mathbf{w}) & \equiv \pi\left(\mathfrak{S}_{\zeta}(\mathbf{v}, \mathbf{w})\right), \quad \text { if } \zeta=-a \omega(\zeta), a(\boldsymbol{\omega} * \mathbf{v})=\mathbf{v}, a \mathbf{w}=\mathbf{w}, \\
\mathfrak{S}_{1}(\mathbf{w}) & =\bigsqcup_{a(\boldsymbol{\omega} * \mathbf{v})=\mathbf{v}} \mathfrak{S}_{1}(\mathbf{v}, \mathbf{w}), \quad \text { if } \zeta=-a \boldsymbol{\omega}(\zeta), a \mathbf{w}=\mathbf{w} .
\end{aligned}
$$

In particular, the proper morphism $\pi$ in (8) restricts to a proper morphism:

$$
\pi^{\sigma}: \mathfrak{S}_{\zeta}(\mathbf{v}, \mathbf{w}) \rightarrow \mathfrak{S}_{1}(\mathbf{v}, \mathbf{w}) \text { and } \pi^{\sigma}: \mathfrak{S}_{\zeta}(\mathbf{w}) \rightarrow \mathfrak{S}_{1}(\mathbf{w})
$$


Let $\mathrm{G}_{\mathbf{w}}^{\sigma}=\left\{\mathrm{f} \in \mathrm{G}_{\mathbf{w}} \mid \mathrm{f}={ }^{a \tau} \mathrm{f}\right\}$. Since $S_{\omega}$ is $\mathrm{G}_{\mathbf{w}}$-equivariant and $\tau_{\zeta}$ and $a$ satisfy $\tau_{\zeta}(\mathrm{f} .[\mathbf{x}])={ }^{\tau} \mathrm{f} . \tau_{\zeta}([\mathbf{x}])$ and $a(\mathrm{f} .[\mathbf{x}])={ }^{a} \mathrm{f}$. $[\mathbf{x}]$ for all $\mathrm{f} \in \mathrm{G}_{\mathbf{w}}$ and $[\mathbf{x}] \in \mathfrak{M}_{\zeta}(\mathbf{v}, \mathbf{w})$, we see that the automorphism $\sigma$ satisfies the following property:

$$
\sigma(\mathrm{f} .[\mathbf{x}])={ }^{a \tau} \mathrm{f} . \sigma([\mathbf{x}]) \quad \forall \mathrm{f} \in \mathrm{G}_{\mathbf{w}},[\mathbf{x}] \in \mathfrak{M}_{\zeta}(\mathbf{v}, \mathbf{w}) .
$$

It induces $\mathrm{G}_{\mathbf{w}}^{\sigma}$-actions on $\mathfrak{S}_{\zeta}(\mathbf{v}, \mathbf{w})$ and $\mathfrak{S}_{1}(\mathbf{v}, \mathbf{w})$, which are compatible with the proper map $\pi^{\sigma}$. There is a natural $\mathbb{C}^{\times}$-action on $\mathbf{M}(\mathbf{v}, \mathbf{w})$ given by $\mathbf{x}=$ $\left(x_{h}, p_{i}, q_{i}\right)_{h \in H, i \in I} \mapsto t . \mathbf{x}=\left(t x_{h}, t p_{i}, t q_{i}\right)_{h \in H, i \in I}$ for all $t \in \mathbb{C}^{\times}$. This $\mathbb{C}^{\times}$-action commutes with the isomorphisms $a$ and $\tau$ on $\mathbf{M}(\mathbf{v}, \mathbf{w})$, which in turn induces a $\mathbb{C}^{\times}$-action on $\mathfrak{S}_{1}(\mathbf{v}, \mathbf{w})$. If the parameter $\zeta_{\mathbb{C}}=0$, then the $\mathbb{C}^{\times}$-action on $\mathbf{M}(\mathbf{v}, \mathbf{w})$ restricts to a $\mathbb{C}^{\times}$-action on $\Lambda_{\zeta \mathbb{C}}(\mathbf{v}, \mathbf{w})$, and then on $\mathfrak{M}_{\zeta}(\mathbf{v}, \mathbf{w})$. This action clearly commutes with the $\mathrm{G}_{\mathbf{w}}^{\sigma}$-actions on $\mathfrak{S}_{\zeta}(\mathbf{v}, \mathbf{w})$ and $\mathfrak{S}_{1}(\mathbf{v}, \mathbf{w})$. In this case the morphism $\pi^{\sigma}$ is $\mathrm{G}_{\mathbf{w}}^{\sigma} \times \mathbb{C}^{\times}$-equivariant. The above analysis yields the following proposition.

Proposition 4.1.8. The map $\pi^{\sigma}$ is $\mathrm{G}_{\mathbf{w}}^{\sigma}$-equivariant. If $\zeta_{\mathbb{C}}=0$, it is $\mathrm{G}_{\mathbf{w}}^{\sigma} \times \mathbb{C}^{\times}$equivariant.

4.2. The $\sigma$-quiver varieties, II: $\zeta=\left(0, \zeta_{\mathbb{C}}\right)$. In this section, we assume that $\Gamma$ is Dynkin and $\zeta=\left(0, \zeta_{\mathbb{C}}\right)$, which is not necessarily generic. We give a definition of $\sigma$-quiver varieties under these assumptions by making use of Lusztig's reflection functor and global versions of the transpose $\tau$ and the diagram isomorphism $a$ defined as follows.

4.2.1. Lusztig's variety $Z_{\mathbf{w}}^{\zeta_{\mathbf{c}}}$. Let $\mathcal{F}$ be the space of paths in the Dynkin graph $\Gamma$. The concatenation operation, $\left(\rho, \rho^{\prime}\right) \mapsto \rho \cdot \rho^{\prime}=\delta_{\mathbf{o}(\rho), \mathbf{i}\left(\rho^{\prime}\right)} \rho \rho^{\prime}$, of the paths defines an associative algebra structure on $\mathcal{F}$. The bar involution on $\Gamma$ defines an antiinvolution on $\mathcal{F}$, which we shall denote by the same notation. Let $\mathbf{i}(f)$ and $\mathbf{o}(f)$ be the ending and starting vertex of the path $f$. Let $[i]$ be the path of length zero such that $\mathbf{i}([i])=\mathbf{o}([i])=i$. For $\zeta_{\mathbb{C}} \in \mathbb{C}^{I}$, let

$$
\theta_{i, \zeta_{\mathbb{C}}}=\sum_{h: \mathbf{i}(h)=i} \varepsilon(h) h \bar{h}-\zeta_{\mathbb{C}}^{(i)}[i] \quad \forall i \in I .
$$

Recall that $W$ is an $I$-graded vector space of dimension $\mathbf{w}$. Let $Z_{\mathbf{w}}^{\zeta_{\mathrm{C}}}$ be the set of linear maps $\pi^{\prime}$ from $\mathcal{F}$ to $\operatorname{End}(W)$ such that

- $\pi^{\prime}(f) \in \operatorname{Hom}\left(W_{\mathbf{o}(f)}, W_{\mathbf{i}(f)}\right) \subseteq \operatorname{End}(W)$ for all path $f$,

- $\pi^{\prime}(f) \pi^{\prime}\left(f^{\prime}\right)=\pi^{\prime}\left(f \cdot \theta_{i, \zeta_{\mathbb{C}}} \cdot f^{\prime}\right)$ for all paths $f$ and $f^{\prime}$ such that $\mathbf{i}\left(f^{\prime}\right)=i=\mathbf{o}(f)$. $Z_{\mathbf{w}}^{\zeta_{\mathbb{C}}}$ is an affine algebraic variety by $\left[\mathrm{L} 00\right.$ ] and isomorphic to $\mathfrak{M}_{\left(0, \zeta_{\mathbb{C}}\right)}(\mathbf{v}, \mathbf{w})$ for $\mathbf{v}$ very large. As a set, $Z_{\mathbf{w}}^{\zeta_{\mathrm{C}}}$ can be identified with $\mathfrak{M}_{0}(\mathbf{w})$ under a proper treatment.

Following [L00, 2.3], there is a $\mathbb{C}^{\times}$-action on the totality $\bigsqcup_{\zeta_{\mathbb{C}} \in \mathbb{C}^{I}} Z_{\mathbf{w}}^{\zeta_{\mathbb{C}}}$ given by $t: Z_{\mathbf{w}}^{\zeta_{\mathbb{C}}} \rightarrow Z_{\mathbf{w}}^{t^{2} \zeta_{\mathbb{C}}}$ and $\left(t . \pi^{\prime}\right)(f)=t^{s+2} \pi^{\prime}(f)$ if $f=h_{1} \cdots h_{s}$ for all $\pi^{\prime} \in Z_{\mathbf{w}}^{\zeta_{\mathbb{C}}}$. Following [L00, 2.4], there is a $\mathrm{G}_{\mathbf{w}}$-action on $Z_{\mathbf{w}}^{\zeta_{\mathbf{C}}}$ by $\left(g . \pi^{\prime}\right)(f)=g_{\mathbf{i}(f)} \pi^{\prime}(f) g_{\mathbf{o}(f)}^{-1}$ for all path $f$ and $\pi^{\prime} \in Z_{\mathbf{w}}^{\zeta_{\mathbb{C}}}$.

4.2.2. The transpose $\tau_{0}$. We extend the orientation function $\varepsilon$ to a function on the set of paths in $\Gamma$ by defining $\varepsilon([i])=1$ and $\varepsilon(f)=\prod_{i=1}^{s} \varepsilon\left(h_{i}\right)$ if $f=h_{1} \cdots h_{s}$. Now assume further that $W$ is an $I$-graded formed space. We define an isomorphism

$$
\tau_{0}: Z_{\mathbf{w}}^{\zeta \mathbb{C}} \rightarrow Z_{\mathbf{w}}^{-\zeta_{\mathbb{C}}}, \quad \pi^{\prime} \mapsto \tau_{0}\left(\pi^{\prime}\right),
$$

where $\tau_{0}\left(\pi^{\prime}\right)(f)=-\varepsilon(f) \pi^{\prime}(\bar{f})^{*}$ for any path $f$ in $\Gamma$. 
We must show that $\tau_{0}$ on $Z_{\mathbf{w}}^{\zeta_{\mathbb{C}}}$ is well-defined, that is, $\tau_{0}\left(\pi^{\prime}\right) \in Z_{\mathbf{w}}^{-\zeta_{\mathbb{C}}}$. Clearly $\tau_{0}\left(\pi^{\prime}\right)(f) \in \operatorname{Hom}\left(W_{\mathbf{o}(f)}, W_{\mathbf{i}(f)}\right)$. For any two paths $f, f^{\prime}$ in $\Gamma$ such that $\mathbf{i}\left(f^{\prime}\right)=i=$ $\mathbf{o}(f)$, we have

$$
\begin{aligned}
\tau_{0}\left(\pi^{\prime}\right)(f) \tau_{0}\left(\pi^{\prime}\right)\left(f^{\prime}\right) & =\varepsilon(f) \varepsilon\left(f^{\prime}\right) \pi^{\prime}(\bar{f})^{*} \pi^{\prime}\left(\overline{f^{\prime}}\right)^{*} \\
& =\varepsilon(f) \varepsilon\left(f^{\prime}\right)\left(\pi^{\prime}\left(\overline{f^{\prime}}\right) \pi^{\prime}(\bar{f})\right)^{*} \\
& =\varepsilon(f) \varepsilon\left(f^{\prime}\right)\left(\pi^{\prime}\left(\overline{f^{\prime}} \theta_{i, \zeta_{\mathbb{C}}} \bar{f}\right)\right)^{*} \\
& =-\varepsilon(f) \varepsilon\left(f^{\prime}\right)\left(\pi^{\prime}\left(\overline{f \theta_{i,-\zeta_{\mathbb{C}}} f^{\prime}}\right)\right)^{*}=\tau_{0}\left(\pi^{\prime}\right)\left(f \theta_{i,-\zeta_{\mathbb{C}}} f^{\prime}\right) .
\end{aligned}
$$

Therefore the well-definedness of $\tau_{0}$ follows.

There is a morphism of varieties $\Lambda_{\zeta_{\mathbb{C}}}(\mathbf{v}, \mathbf{w}) \rightarrow Z_{\mathbf{w}}^{\zeta_{\mathbb{C}}}$ sending a point $\mathbf{x}=\left(x_{h}, p_{i}, q_{i}\right)$ to $\pi^{\prime}$ such that $\pi^{\prime}(f)=q_{\mathbf{i}\left(h_{1}\right)} x_{h_{1}} \cdots x_{h_{s-1}} x_{h_{s}} p_{\mathbf{o}\left(h_{s}\right)}$ for any path $f=h_{1} \cdots h_{s}$. The morphism then induces an immersion $\vartheta: \mathfrak{M}_{\left(0, \zeta_{\mathbb{C}}\right)}(\mathbf{v}, \mathbf{w}) \rightarrow Z_{\mathbf{w}}$. It is clear that the isomorphism $\tau$ on $\Lambda_{\zeta_{\mathbb{C}}}(\mathbf{v}, \mathbf{w})$ in (16) is compatible with the isomorphism $\tau_{0}$ on $Z_{\mathbf{w}}^{\zeta_{\mathbb{C}}}$. This implies that the isomorphisms $\tau_{0}$ on $\mathfrak{M}_{\left(0, \zeta_{\mathbb{C}}\right)}(\mathbf{v}, \mathbf{w})$ and $Z_{\mathbf{w}}^{\zeta_{\mathbb{C}}}$ are compatible under the immersion $\vartheta$, that is, the following diagram commutes:

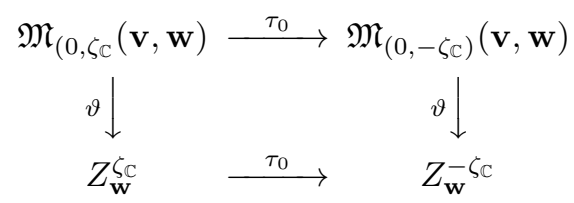

This indicates that the notation $\tau_{0}$ on $Z_{\mathbf{w}}^{\zeta_{\mathbb{C}}}$ and $\mathfrak{M}_{\left(0, \zeta_{\mathbb{C}}\right)}(\mathbf{v}, \mathbf{w})$ will not cause any confusion.

Remark 4.2.3. We have $\left(Z_{\mathbf{w}}^{0}\right)^{\tau_{0}}=\left\{\pi^{\prime} \in Z_{\mathbf{w}}^{0} \mid \pi^{\prime}(f)=-\varepsilon(f) \pi^{\prime}(\bar{f})^{*}\right.$ for any path $f$ in $\left.\Gamma\right\}$. This description is similar to the definition of classical Lie algebras in (80).

4.2.4. Diagram isomorphism $\Theta_{a, \varepsilon}$. Retaining the setting in Section 3.3 the automorphism $a$ on $\Gamma$ naturally induces an automorphism, still denoted by $a$, on $\mathcal{F}$ such that $a: i \mapsto a(i)$ and $a: h \mapsto a(h)$. We define another automorphism on $\mathcal{F}$ by rescaling $a^{-1}$ on $\mathcal{F}$ :

$$
\Phi_{a, \varepsilon}: \mathcal{F} \rightarrow \mathcal{F}, \quad[i] \mapsto a^{-1}([i]), h \mapsto \varepsilon(h)^{\frac{1-c}{2}} a^{-1}(h) \quad \forall i \in I, h \in H .
$$

This is an algebra homomorphism, due to the multiplicative property of $\varepsilon: \varepsilon\left(f f^{\prime}\right)=$ $\varepsilon(f) \varepsilon\left(f^{\prime}\right)$ if $f$ and $f^{\prime}$ are two paths such that $\mathbf{i}\left(f^{\prime}\right)=\mathbf{o}(f)$. Let $a^{-1}\left(\zeta_{\mathbb{C}}\right)$ be the tuple whose $i$ th entry is $\zeta_{\mathbb{C}}^{(a(i))}$. The reason why we define $\Phi_{a, \varepsilon}$ this way is due to the following identity:

$$
\Phi_{a, \varepsilon}\left(\theta_{i, \zeta_{\mathbb{C}}}\right)=\theta_{a^{-1}(i), a^{-1}\left(\zeta_{\mathbb{C}}\right)}^{\forall i \in I}
$$

Indeed, we have

$$
\begin{aligned}
\Phi_{a, \varepsilon}\left(\theta_{i, \zeta_{\mathbb{C}}}\right) & =\sum_{\mathbf{i}(h)=i} \varepsilon(h)(-1)^{\frac{1-c}{2}} a^{-1}(h) a^{-1}(\bar{h})-\zeta_{\mathbb{C}}^{(i)}\left[a^{-1}(i)\right] \\
& =\sum_{\mathbf{i}(h)=i} \varepsilon\left(a^{-1}(h)\right) a^{-1}(h) a^{-1}(\bar{h})-a^{-1}\left(\zeta_{\mathbb{C}}\right)^{\left(a^{-1}(i)\right)}\left[a^{-1}(i)\right]=\theta_{a^{-1}(i), a^{-1}\left(\zeta_{\mathbb{C}}\right)} .
\end{aligned}
$$

Recall that $a(W)$ is the $I$-graded space whose $i$ th component is $W_{a^{-1}(i)}$. We then have an isomorphism of vector spaces by permutation $s_{a}: W \rightarrow a(W)$ so 
that $s_{a}(w)_{i}=w_{a^{-1}(i)}$, where $w=\left(w_{i}\right)_{i \in I} \in W$. This isomorphism defines an isomorphism

$$
r_{a}: \operatorname{End}(W) \rightarrow \operatorname{End}(a(W)), \phi \mapsto r_{a}(\phi):=s_{a} \circ \phi \circ s_{a}^{-1} .
$$

Let

$$
\Theta_{a, \varepsilon}: Z_{\mathbf{w}}^{\zeta_{\mathbb{C}}} \rightarrow Z_{a^{-1}\left(\zeta_{\mathbb{w}}\right)}^{a^{-1}}
$$

be the isomorphism defined by $\Theta_{a, \varepsilon}\left(\pi^{\prime}\right)=r_{a^{-1}} \circ \pi^{\prime} \circ \Phi_{a, \varepsilon}^{-1} \forall \pi^{\prime} \in Z_{\mathbf{w}}^{\zeta_{\mathrm{C}}}$. Due to (33), $\Theta_{a, \varepsilon}$ is well-defined. $\Theta_{a, \varepsilon}$ is compatible with the diagram isomorphism $a^{-1}$ on $\mathfrak{M}_{\left(0, \zeta_{\mathbb{C}}\right)}(\mathbf{v}, \mathbf{w})$ in (20).

Lemma 4.2.5. There is a commutative diagram

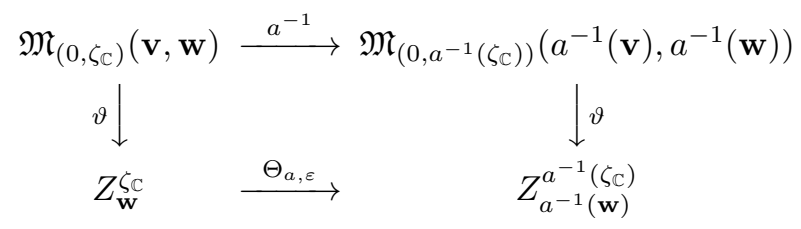

Proof. Let $[\mathbf{x}]=[x, p, q] \in \mathfrak{M}_{\left(0, \zeta_{\mathbb{C}}\right)}(\mathbf{v}, \mathbf{w})$. It suffices to show that $\vartheta \circ a^{-1}(\mathbf{x})=$ $\Theta_{a, \varepsilon} \circ \vartheta(\mathbf{x})$. Given any arrow $h$, the evaluations of the left- and right-hand sides on $h$ are equal to $q_{a(\mathbf{i}(h))} x_{a(h)} p_{a(\mathbf{o}(h))}$. So the equality must hold, and the lemma follows.

4.2.6. Lusztig's reflection functor on $Z_{\mathbf{w}}^{\zeta_{\mathbb{C}}}$. Lusztig [L00] defined a reflection functor

$$
S_{i}: Z_{\mathbf{w}}^{\zeta_{\mathbb{C}}} \rightarrow Z_{\mathbf{w}}^{s_{i}\left(\zeta_{\mathbb{C}}\right)}, \pi^{\prime} \mapsto S_{i}\left(\pi^{\prime}\right),
$$

where the evaluation of $S_{i}\left(\pi^{\prime}\right)$ on a given path $f$ is defined to be

$S_{i}\left(\pi^{\prime}\right)(f)= \begin{cases}\pi^{\prime}([j])+\delta_{j, i} \zeta_{\mathbb{C}}^{(i)} \operatorname{id}_{W_{i}}, & \text { if } f=[j], \\ \sum_{J: J \subset J_{0}}\left(\prod_{t \in J}-\varepsilon\left(h_{t}\right) \zeta_{\mathbb{C}}^{(i)}\right) \pi^{\prime}\left(\left(h_{1} \cdots h_{s}\right)^{\vee J}\right), & \text { if } f=h_{1} \cdots h_{s}, s \geq 1 .\end{cases}$

Here $J_{0}=\left\{t \in[2, r] \mid \mathbf{i}\left(h_{t-1}\right)=i=\mathbf{o}\left(h_{t}\right)\right\}$ and the superscript $\vee J$ is the operation of removing the arrows $h_{t-1}, h_{t}$ for all $t \in J$. (Note that $J$ can be an empty set.) Since the isomorphism $S_{i}$ satisfies the Weyl group relations, we define $S_{\omega}=S_{i_{1}} \cdots S_{i_{s}}$ for any $\omega=s_{i_{1}} \cdots s_{i_{s}} \in \mathcal{W}$.

4.2.7. The $\sigma$-quiver variety $\mathfrak{S}_{\left(0, \zeta_{\mathbb{C}}\right)}(\mathbf{w})$. Let

$$
\sigma_{0}=S_{\omega} \circ \Theta_{a, \varepsilon} \circ \tau_{0}: Z_{\mathbf{w}}^{\zeta_{\mathbb{C}}} \rightarrow Z_{a^{-1}(\mathbf{w})}^{-\omega a^{-1}\left(\zeta_{\mathbb{C}}\right)} .
$$

When the isomorphism $\sigma_{0}$ becomes an automorphism, we can take its fixed-point.

Definition 4.2.8. $\mathfrak{S}_{\left(0, \zeta_{\mathbb{C}}\right)}(\mathbf{w})=\left(Z_{\mathbf{w}}^{\zeta_{\mathbb{C}}}\right)^{\sigma_{0}}$, if $\mathbf{w}=a^{-1}(\mathbf{w}), \zeta_{\mathbb{C}}=-\omega a^{-1}\left(\zeta_{\mathbb{C}}\right)$.

When there is no danger of confusion, we use $\mathfrak{S}_{0}(\mathbf{w})$ for $\mathfrak{S}_{(0, \zeta)}(\mathbf{w})$. By (31), Lemma 4.2.5, and [L00, the definition is compatible with the varieties $\mathfrak{S}_{\zeta}(\mathbf{v}, \mathbf{w})$ with $a$ replaced by $a^{-1}$, and so we have proper morphisms

$$
\pi^{\sigma}: \mathfrak{S}_{\zeta}(\mathbf{v}, \mathbf{w}) \rightarrow \mathfrak{S}_{0}(\mathbf{w}), \quad \pi^{\sigma}: \mathfrak{S}_{\zeta}(\mathbf{w}) \rightarrow \mathfrak{S}_{0}(\mathbf{w}) .
$$

There is a $G_{\mathbf{w}}^{\sigma}$-action on $\mathfrak{S}_{0}(\mathbf{w})$ induced from $Z_{\mathbf{w}}^{\zeta_{\mathbb{C}}}$, and further a $G_{\mathbf{w}}^{\sigma} \times \mathbb{C}^{\times}$-action on $\mathfrak{S}_{0}(\mathbf{w})$ if $\zeta_{\mathbb{C}}=0$. It is clear that the morphisms in (36) are $\mathrm{G}_{\mathbf{w}}^{\sigma}$-equivariant (resp. $\mathrm{G}_{\mathbf{w}}^{\sigma} \times \mathbb{C}^{\times}$-equivariant if $\zeta_{\mathbb{C}}=0$ ). It is also clear that $\pi^{\sigma}$ factors through the map under the same notation $\pi^{\sigma}$ in (27) and $\mathfrak{S}_{1}(\mathbf{v}, \mathbf{w})$ is a closed subvariety of $\mathfrak{S}_{\left(0, \zeta_{\mathbb{C}}\right)}(\mathbf{w})$. 
Remark 4.2.9. We can define $\mathfrak{S}_{0}(\mathbf{v}, \mathbf{w})=\mathfrak{M}_{0}(\mathbf{v}, \mathbf{w}) \cap \mathfrak{S}_{0}(\mathbf{w})$ in corresponding to $\mathfrak{S}_{\zeta}(\mathbf{v}, \mathbf{w})$. This definition makes sense even when $\Gamma$ is not Dynkin, but in this generality we are not sure if $\mathfrak{S}_{0}(\mathbf{v}, \mathbf{w})$ is an algebraic variety. On the other hand, we can always define the fixed-point locus $\mathfrak{M}_{0}(\mathbf{v}, \mathbf{w})^{a \tau}$ as long as $a(\mathbf{v})=\mathbf{v}$ and $a(\mathbf{w})=\mathbf{w}$. This fixed-point locus does not have to assume $\Gamma$ being Dynkin either.

Lemma 4.2.10. When $\zeta_{\mathbb{C}}=0$ and $\omega * \mathbf{v}=\mathbf{v}, \mathfrak{M}_{0}(\mathbf{v}, \mathbf{w})^{a \tau}=\mathfrak{S}_{0}(\mathbf{v}, \mathbf{w})$.

Proof. In this case the reflection functor is the identity morphism by [L00].

4.3. Weyl group action on $\sigma$-quiver varieties. Let $\zeta$ be generic in this section. The diagram automorphism $a$ induces an automorphism on the Weyl group $\mathcal{W}$. Let $\mathcal{W}^{\omega, a}=\{x \in \mathcal{W} \mid x \omega=\omega x, a(x)=x\}$. This implies that the action $S_{x}$ for $x \in \mathcal{W}^{\omega, a}$ on quiver varieties commutes with the action $S_{\omega}$ and $a$. Further, thanks to Lemma 3.2.3, it commutes with the isomorphism $\sigma$. Hence we have the following proposition.

Proposition 4.3.1. The action $S_{x}$ for $x \in \mathcal{W}^{\omega, a}$ restricts to an action on $\sigma$-quiver varieties:

$$
S_{x}^{\sigma}: \mathfrak{S}_{\zeta}(\mathbf{v}, \mathbf{w}) \rightarrow \mathfrak{S}_{x(\zeta)}(x * \mathbf{v}, \mathbf{w}) \quad \forall x \in \mathcal{W}^{\omega, a} .
$$

As a consequence, we obtain the following corollary.

Corollary 4.3.2. The group $\mathcal{W}^{\omega, a}$ acts on the cohomology group $\mathrm{H}^{*}\left(\mathfrak{S}_{\zeta}(\mathbf{v}, \mathbf{w}), \mathbb{Z}\right)$ when $\mathbf{w}-\mathbf{C v}=0$.

Remark 4.3.3. When $\Gamma$ is of Dynkin type, $a=1$ and $\omega=w_{0}$, the group $\mathcal{W}^{\omega, a}$ is a Weyl group of type $B_{\ell}$ if $\Gamma$ is of type $A_{2 \ell}, C_{\ell}$ if $\Gamma$ is of type $A_{2 \ell-1}, B_{\ell}$ if $\Gamma$ is of type $D_{\ell+1}, \ell$ even, or of type $F_{4}$ if $\Gamma$ is of type $E_{6}$. If $\Gamma$ is of type $D_{4}, \omega=w_{0}$, and $a$ is the unique automorphism of order 3 , then the group $\mathcal{W}^{\omega, a}$ is the Weyl group $G_{2}$.

4.4. Symplectic structure on $\mathfrak{S}_{\zeta}(\mathbf{v}, \mathbf{w})$. In this section, we assume that the parameter $\zeta$ is generic. Recall the symplectic vector space $\mathbf{M}(\mathbf{v}, \mathbf{w})$ from Section 2.2 . It is straightforward to check that the isomorphisms $a$ and $\tau$ on $\mathbf{M}(\mathbf{v}, \mathbf{w})$ in (20) and (15), respectively, are symplectomorphisms. The varieties $\mathfrak{M}_{\zeta}(\mathbf{v}, \mathbf{w})$ inherit from $\mathbf{M}(\mathbf{v}, \mathbf{w})$ a symplectic structure. In turn, the fact that $a$ and $\tau$ being symplectomorphisms implies that the induced isomorphisms $a$ and $\tau_{\zeta}$ on $\mathfrak{M}_{\zeta}(\mathbf{v}, \mathbf{w})$ are also symplectomorphisms. By the analysis in [N03, Theorem 6.1], the reflection functor $S_{\omega}$ is a hyper-Kähler isometry and in particular a symplectomorphism. Altogether, we see that the isomorphism $\sigma$ on $\mathfrak{M}_{\zeta}(\mathbf{v}, \mathbf{w})$ is a symplectomorphism.

Proposition 4.4.1. Assume that $W$ is a $\delta_{\mathbf{w}}$-formed space and the order of $\omega$ is finite. Then the $\sigma$-quiver variety $\mathfrak{S}_{\zeta}(\mathbf{v}, \mathbf{w})$ is a symplectic submanifold of $\mathfrak{M}_{\zeta}(\mathbf{v}, \mathbf{w})$.

Proof. We only need to show that the restriction of the form $\omega$ to $\mathfrak{S}_{\zeta}(\mathbf{v}, \mathbf{w})$ is nondegenerate. Fix a point $[\mathbf{x}] \in \mathfrak{S}_{\zeta}(\mathbf{v}, \mathbf{w})$; the differential $d \sigma_{[\mathbf{x}]}$ of the automorphism $\sigma$ at $[\mathbf{x}]$ is an automorphism on the tangent space $T_{[\mathbf{x}]} \mathfrak{M}_{\zeta}(\mathbf{v}, \mathbf{w})$. By the assumption, we see from Proposition 4.1.5 that $\sigma^{N}=1$ for some $N$, and hence $\left(d \sigma_{[\mathbf{x}]}\right)^{N}=1$. By a result of Edixhoven [E92, the fixed points of $d \sigma_{[\mathbf{x}]}$, i.e., the eigenspace of eigenvalue 1 , is exactly the tangent space $T_{[\mathbf{x}]} \mathfrak{S}_{\zeta}(\mathbf{v}, \mathbf{w})$ of $\mathfrak{S}_{\zeta}(\mathbf{v}, \mathbf{w})$ at $[x]$. It thus yields the following eigenspace decomposition:

$$
T_{[\mathbf{x}]} \mathfrak{M}_{\zeta}(\mathbf{v}, \mathbf{w})=T_{[\mathbf{x}]} \mathfrak{S}_{\zeta}(\mathbf{v}, \mathbf{w}) \oplus C,
$$


where $C$ consists of linear combinations of eigenvectors of eigenvalues other than 1. By the above analysis, the automorphism $\sigma$ is a symplectomorphism, and so this implies that $T_{[\mathbf{x}]} \mathfrak{S}_{\zeta}(\mathbf{v}, \mathbf{w})$ and $C$ are orthogonal with each other. Hence the restriction of the symplectic form on them are nondegenerate. We are done.

Since the zero fiber $\pi^{-1}(0)$ is Lagrangian, we see that the fiber $\left(\pi^{\sigma}\right)^{-1}(0)=$ $\pi^{-1}(0)^{\sigma}$ is isotropic. But the fiber $\left(\pi^{\sigma}\right)^{-1}(0)$ is not coisotropic and hence not Lagrangian in general; see Remark 6.2.2(3). A nice consequence of Proposition 4.4.1 is the semismallness of $\pi^{\sigma}$.

Corollary 4.4.2. The map $\pi^{\sigma}: \mathfrak{S}_{\zeta}(\mathbf{v}, \mathbf{w}) \rightarrow \mathfrak{S}_{1}(\mathbf{v}, \mathbf{w})$ is semismall.

Proof. By [N98, Theorem 7.2], the fiber product $\mathfrak{M}_{\zeta}(\mathbf{v}, \mathbf{w}) \times_{\mathfrak{M}_{0}(\mathbf{v}, \mathbf{w})} \mathfrak{M}_{\zeta}(\mathbf{v}, \mathbf{w})$ is a lagrangian subvariety in $\mathfrak{M}_{\zeta}(\mathbf{v}, \mathbf{w}) \times \mathfrak{M}_{\zeta}(\mathbf{v}, \mathbf{w})$. So its $\sigma$-analogue $\mathfrak{S}_{\zeta}(\mathbf{v}, \mathbf{w}) \times_{\mathfrak{S}_{1}(\mathbf{v}, \mathbf{w})}$ $\mathfrak{S}_{\zeta}(\mathbf{v}, \mathbf{w})$ is isotropic in $\mathfrak{S}_{\zeta}(\mathbf{v}, \mathbf{w}) \times \mathfrak{S}_{\zeta}(\mathbf{v}, \mathbf{w})$, and thus has at most half of the dimension of the latter manifold. So we have

$$
\operatorname{dim} \mathfrak{S}_{\zeta}(\mathbf{v}, \mathbf{w}) \times_{\mathfrak{S}_{1}(\mathbf{v}, \mathbf{w})} \mathfrak{S}_{\zeta}(\mathbf{v}, \mathbf{w})=\operatorname{dim} \mathfrak{S}_{\zeta}(\mathbf{v}, \mathbf{w}) .
$$

According to [CG, 8.9.2], it implies the corollary.

Remark 4.4.3.

(1) We refer to Remark 5.3 .2 for an alternative proof of Corollary 4.4 .2

(2) By Corollary 4.4.2, to show that $\mathfrak{S}_{\zeta}(\mathbf{v}, \mathbf{w})$ is equidimensional, a.k.a., of pure dimension, it is enough to show that the images of all connected components under $\pi^{\sigma}$ coincide.

\section{QUiver VARIETIES AND SYMMETRIC PAIRS}

In this section, we assume that the graph $\Gamma$ is a Dynkin diagram, $|a|=1$ or 2 , the Weyl group element $\omega=w_{0}$ is the longest element in the Weyl group of $\Gamma$, and the sign function $\delta_{\mathbf{w}}$ is $\Gamma$-alternating in the definition of the automorphism $\sigma$. In this case, we have $\sigma^{2}=1$ by Proposition 4.1.5.

5.1. Restriction diagram. Now we assume that $\zeta_{\mathbb{C}}=0$ and $\xi_{i}=1$ for all $i \in I$.

Let $\mathrm{T}$ be a torus in $\mathrm{G}_{\mathbf{w}}$. Let $\mathfrak{M}_{\zeta}(\mathbf{v}, \mathbf{w})^{\mathrm{T}}$ be the T-fixed-point subvariety of $\mathfrak{M}_{\zeta}(\mathbf{v}, \mathbf{w})$. For each homomorphism $\rho: \mathrm{T} \rightarrow \mathrm{G}_{\mathbf{v}}$, let

$$
\mathfrak{M}_{\zeta}(\rho)=\left\{[\mathbf{x}] \in \mathfrak{M}_{\zeta}(\mathbf{v}, \mathbf{w}) \mid t \cdot \mathbf{x}=\rho(t)^{-1} \cdot \mathbf{x} \forall t \in \mathrm{T}\right\} .
$$

Nakajima N00 showed that the $\mathfrak{M}_{\zeta}(\rho)$ depends on the $\mathbf{G}_{\mathbf{v}}$-conjugacy class of $\rho$ and there is a partition of $\mathfrak{M}_{\zeta}(\mathbf{v}, \mathbf{w})^{\mathrm{T}}$ into connected components: $\mathfrak{M}_{\zeta}(\mathbf{v}, \mathbf{w})^{\mathrm{T}}=$ $\coprod \mathfrak{M}_{\zeta}(\rho)$, where the union is over the set of all $\mathbf{G}_{\mathbf{v}}$-conjugacy classes, say $\langle\rho\rangle$, of homomorphisms $\rho: \mathrm{T} \rightarrow \mathrm{G}_{\mathbf{v}}$. We define

$$
\mathfrak{S}_{\zeta}(\mathbf{v}, \mathbf{w})^{\mathrm{T}}=\mathfrak{S}_{\zeta}(\mathbf{v}, \mathbf{w}) \cap \mathfrak{M}_{\zeta}(\mathbf{v}, \mathbf{w})^{\mathrm{T}} .
$$

Thus there is a decomposition

$$
\mathfrak{S}_{\zeta}(\mathbf{v}, \mathbf{w})^{\mathrm{T}}=\coprod_{\langle\rho\rangle} \mathfrak{S}_{\zeta}(\rho), \quad \mathfrak{S}_{\zeta}(\rho)=\mathfrak{S}_{\zeta}(\mathbf{v}, \mathbf{w}) \cap \mathfrak{M}_{\zeta}(\rho) .
$$

In the special case $\mathrm{T}=\mathbb{C}^{\times} \subseteq \mathrm{G}_{\mathrm{w}}^{\sigma}$, we can further define

$$
\begin{aligned}
\mathfrak{S}_{\zeta}(\mathbf{v}, \mathbf{w})^{+\mathbb{C}^{\times}} & =\left\{[\mathbf{x}] \in \mathfrak{S}_{\zeta}(\mathbf{v}, \mathbf{w}) \mid \lim _{t \rightarrow 0} t .[\mathbf{x}] \text { exists }\right\} \\
\mathfrak{S}_{\zeta}(\mathbf{v}, \mathbf{w})^{-\mathbb{C}^{\times}} & =\left\{[\mathbf{x}] \in \mathfrak{S}_{\zeta}(\mathbf{v}, \mathbf{w}) \mid \lim _{t \rightarrow \infty} t .[\mathbf{x}] \text { exists }\right\} .
\end{aligned}
$$


Similarly, there are varieties $\mathfrak{M}_{\zeta}(\mathbf{v}, \mathbf{w})^{ \pm \mathbb{C}^{\times}}$. Since the chosen $\mathbb{C}^{\times}$is in $G_{\mathbf{w}}^{\sigma}$, we have

$$
\mathfrak{S}_{\zeta}(\mathbf{v}, \mathbf{w})^{ \pm \mathbb{C}^{\times}}=\mathfrak{S}_{\zeta}(\mathbf{v}, \mathbf{w}) \cap \mathfrak{M}_{\zeta}(\mathbf{v}, \mathbf{w})^{ \pm \mathbb{C}^{\times}}
$$

Thus there is the following hyperbolic localization/restriction diagram:

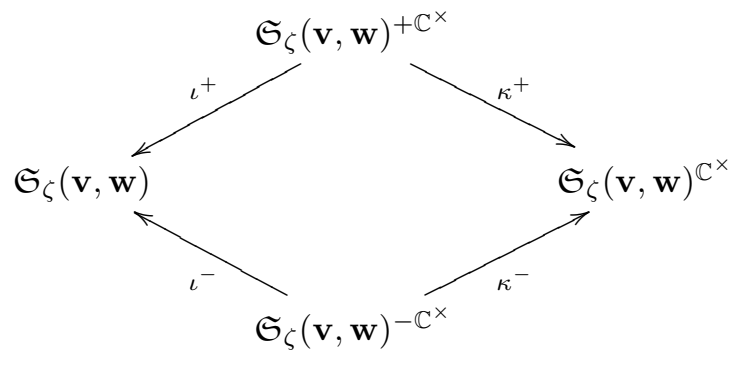

where $\iota^{ \pm}$and $\kappa^{ \pm}$are natural embeddings and projections.

5.2. A characterization of $\mathfrak{S}_{\zeta}(\mathbf{v}, \mathbf{w})^{\mathrm{T}}$. Assume now that we have a decomposition $W=W^{1} \oplus W^{2} \oplus W^{3}$ of the formed space $W$ such that the following conditions hold:

- For all $i \in I$, the restrictions of the form to $W_{i}^{1}$ and $W_{i}^{2} \oplus W_{i}^{3}$ are nondegenerate.

- For all $i \in I, W_{i}^{1}$ and $W_{i}^{2} \oplus W_{i}^{3}$ are orthogonal to each other.

- For all $i \in I$, we have $\left(W_{i}^{2}\right)_{W_{i}^{2} \oplus W_{i}^{3}}^{\perp}=W_{i}^{3},\left(W_{i}^{3}\right)_{W_{i}^{2} \oplus W_{i}^{3}}^{\perp}=W_{i}^{2}$, where $(-)_{W_{i}^{2} \oplus W_{i}^{3}}^{\perp}$ is taken in $W_{i}^{2} \oplus W_{i}^{3}$, and hence $W_{i}^{2}$ and $W_{i}^{3}$ are maximal isotropic in $W_{i}^{2} \oplus W_{i}^{3}$ of the same dimension.

- $a\left(W^{1}\right)=W^{1}, a\left(W^{2}\right)=W^{2}$, and $a\left(W^{3}\right)=W^{3}$.

Set $\operatorname{dim} W^{1}=\mathbf{w}^{1}, \operatorname{dim} W^{2}=\mathbf{w}^{2}$, and $\operatorname{dim} W^{3}=\mathbf{w}^{3}$ so that they satisfy the condition as follows from the above assumption:

$$
\mathbf{w}^{2}=\mathbf{w}^{3}, \mathbf{w}^{2}=a \mathbf{w}^{2}, a \mathbf{w}^{1}=\mathbf{w}^{1}, \text { and } \mathbf{w}=\mathbf{w}^{1}+2 \mathbf{w}^{2} .
$$

Consider the following 1-parameter subgroup in $\mathrm{G}_{\mathbf{w}}^{\sigma}$ :

$$
\lambda: \mathbb{C}^{\times} \rightarrow \mathrm{G}_{\mathbf{w}}^{\sigma}, \quad t \mapsto \mathrm{id}_{W^{1}} \oplus t \cdot \mathrm{id}_{W^{2}} \oplus t^{-1} \cdot \mathrm{id}_{W^{3}} .
$$

By a result [VV00, Lemma 4.4] of Varagnolo and Vasserot, we have that $\mathfrak{M}_{\zeta}(\rho)$ is empty unless $\rho$ is $\mathbf{G}_{\mathbf{v}}$-conjugate to the group homomorphism

$$
\mathbb{C}^{\times} \rightarrow \mathrm{G}_{\mathbf{v}}, \quad t \mapsto \mathrm{id}_{V^{1}} \oplus t \cdot \mathrm{id}_{V^{2}} \oplus t^{-1} \cdot \mathrm{id}_{V^{3}},
$$

for some decomposition $V=V^{1} \oplus V^{2} \oplus V^{3}$. Moreover, if $\rho$ is of the latter form with the dimension vector of $V^{1}, V^{2}$, and $V^{3}$ being $\mathbf{v}^{1}, \mathbf{v}^{2}$, and $\mathbf{v}^{3}$, respectively, then we have

$$
\mathfrak{M}_{\zeta}(\rho)=\mathfrak{M}_{\zeta}\left(\mathbf{v}^{1}, \mathbf{w}^{1}\right) \times \mathfrak{M}_{\zeta}\left(\mathbf{v}^{2}, \mathbf{w}^{2}\right) \times \mathfrak{M}_{\zeta}\left(\mathbf{v}^{3}, \mathbf{w}^{3}\right) .
$$

Since $\lambda\left(\mathbb{C}^{\times}\right) \leq \mathrm{G}_{\mathbf{w}}^{\tau} \cap \mathrm{G}_{\mathbf{w}}^{a} \leq \mathrm{G}_{\mathbf{w}}^{\sigma}$ and $\sigma$ is $\mathrm{G}_{\mathbf{w}}^{\sigma}$-equivariant, we see that

$$
\sigma\left(\mathfrak{M}_{\zeta}(\mathbf{v}, \mathbf{w})^{\lambda\left(\mathbb{C}^{\times}\right)}\right) \subseteq \mathfrak{M}_{\zeta}\left(a w_{0} * \mathbf{v}, a \mathbf{w}\right)^{\lambda\left(\mathbb{C}^{\times}\right)} .
$$

Recall that the automorphism $\sigma$ is a composition $a \tau S_{w_{0}}$. If $\rho$ is of the form (43), we write $w_{0}(\rho)$ to be the group homomorphism

$$
\mathbb{C}^{\times} \rightarrow \mathrm{G}_{\mathbf{v}}, \quad t \mapsto \mathrm{id}_{w_{0}{ }_{\mathbf{w}^{1}} V^{1}} \oplus t \cdot \mathrm{id}_{w_{0} *_{\mathbf{w}^{2}} V^{2}} \oplus t^{-1} \cdot \mathrm{id}_{w_{0}{ }_{\mathbf{w}^{2}} V^{3}},
$$


where $w_{0} *_{\mathbf{w}^{1}} V^{1}, w_{0} *_{\mathbf{w}^{2}} V^{2}$, and $w_{0} *_{\mathbf{w}^{2}} V^{3}$ are vector spaces of dimension vectors $w_{0} *_{\mathbf{w}^{1}} \mathbf{v}^{1}, w_{0} *_{\mathbf{w}^{2}} \mathbf{v}^{2}$, and $w_{0} *_{\mathbf{w}^{2}} \mathbf{v}^{3}$, respectively. Since the construction of the automorphism $\sigma$ is independent of the choice of forms on $V$, we can, and shall, assume that the nondegenerate symmetric form on $V$ has its restriction to $V^{1}, V^{2}$, and $V^{3}$ nondegenerate and that the latter spaces are orthogonal with each other. Note that $a\left(\lambda\left(\mathbb{C}^{\times}\right)\right)=\lambda\left(\mathbb{C}^{\times}\right)$and $\tau\left(\lambda\left(\mathbb{C}^{\times}\right)\right)=\lambda\left(\mathbb{C}^{\times}\right)$. We observe that

$a\left(\mathfrak{M}_{\zeta}(\rho)\right) \subseteq \mathfrak{M}_{a \zeta}\left(a \circ \rho \circ a^{-1}\right), \tau\left(\mathfrak{M}_{\zeta}(\rho)\right) \subseteq \mathfrak{M}_{-\zeta}\left(\tau \circ \rho \circ \tau^{-1}\right), S_{w_{0}}\left(\mathfrak{M}_{\zeta}(\rho)\right) \subseteq \mathfrak{M}_{w_{0} \zeta}\left(w_{0}(\rho)\right)$.

Thus we have $\sigma\left(\mathfrak{M}_{\zeta}(\rho)\right) \subseteq \mathfrak{M}_{-a w_{0} \zeta}\left(w_{0}\left({ }^{a \tau} \rho\right)\right)$, where ${ }^{a \tau} \rho$ is the composition of $\rho$ with the automorphism $a \tau$ on $\mathbf{G}_{\mathbf{v}}$. This implies that $\mathfrak{S}_{\zeta}(\rho)$ is empty unless

$$
\rho=w_{0}\left({ }^{a \tau} \rho\right), \quad \text { up to a } \mathbf{G}_{\mathbf{v}} \text {-conjugate. }
$$

By comparing (45) for $w_{0}\left({ }^{a \tau} \rho\right)$ and (43), we see that $\mathfrak{S}_{\zeta}(\rho)$ is empty unless

$$
\mathbf{v}^{1}=a\left(w_{0} *_{\mathbf{w}^{1}} \mathbf{v}^{1}\right) \text { and } \mathbf{v}^{2}=a\left(w_{0} *_{\mathbf{w}^{3}} \mathbf{v}^{3}\right) .
$$

Assume now that the condition (47) holds. If $\left[\mathbf{x}^{2}\right] \in \mathfrak{M}_{\zeta}\left(\mathbf{v}^{2}, \mathbf{w}^{2}\right)$, then a slight generalization of the operation $\sigma$ yields an element in $\mathfrak{M}_{\zeta}\left(\mathbf{v}^{3}, \mathbf{w}^{3}\right)$, denoted abusively by $\sigma\left(\left[\mathbf{x}^{2}\right]\right)$. (The involution $\tau$ in the definition is changed to be an isomorphism $\mathfrak{M}_{\zeta}\left(\mathbf{v}^{2}, \mathbf{w}^{2}\right) \rightarrow \mathfrak{M}_{-\zeta}\left(\mathbf{v}^{2}, \mathbf{w}^{3}\right)$ with respect to the above decomposition.) Similarly, we can define $\sigma\left(\left[\mathbf{x}^{3}\right]\right)$. By definition, if $\left(\left[\mathbf{x}^{1}\right],\left[\mathbf{x}^{2}\right],\left[\mathbf{x}^{3}\right]\right) \in \mathfrak{M}_{\zeta}(\rho)$ under the identification (44), then

$$
\sigma\left(\left[\mathbf{x}^{1}\right],\left[\mathbf{x}^{2}\right],\left[\mathbf{x}^{3}\right]\right)=\left(\sigma\left(\left[\mathbf{x}^{1}\right]\right), \sigma\left(\left[\mathbf{x}^{3}\right]\right), \sigma\left(\left[\mathbf{x}^{2}\right]\right)\right) .
$$

Thus, in light of the fact that $\sigma^{2}=1,\left(\left[\mathbf{x}^{1}\right],\left[\mathbf{x}^{2}\right],\left[\mathbf{x}^{3}\right]\right) \in \mathfrak{S}_{\zeta}(\rho)$ if and only if $\left[\mathbf{x}^{1}\right]=\sigma\left(\left[\mathbf{x}^{1}\right]\right)$ and $\left[\mathbf{x}^{2}\right]=\sigma\left(\left[\mathbf{x}^{3}\right]\right)$. Therefore, under the assumption (47), it yields

$$
\mathfrak{S}_{\zeta}(\rho) \cong \mathfrak{S}_{\zeta}\left(\mathbf{v}^{1}, \mathbf{w}^{1}\right) \times \mathfrak{M}_{\zeta}\left(\mathbf{v}^{2}, \mathbf{w}^{2}\right)
$$

Summing up the above analysis, there is the following proposition.

Proposition 5.2.1. Assume that $\mathrm{T}=\lambda\left(\mathbb{C}^{\times}\right)$in (42). Then there is an isomorphism

$$
\mathfrak{S}_{\zeta}(\mathbf{v}, \mathbf{w})^{\lambda\left(\mathbb{C}^{\times}\right)} \cong \coprod_{\left(\mathbf{v}^{1}, \mathbf{v}^{2}\right)} \mathfrak{S}_{\zeta}\left(\mathbf{v}^{1}, \mathbf{w}^{1}\right) \times \mathfrak{M}_{\zeta}\left(\mathbf{v}^{2}, \mathbf{w}^{2}\right)
$$

where $\mathbf{w}=\mathbf{w}^{1}+2 \mathbf{w}^{2}, a \mathbf{w}=\mathbf{w}$, a $\mathbf{w}^{1}=\mathbf{w}^{1}$, and the union is over $\left(\mathbf{v}^{1}, \mathbf{v}^{2}\right)$ such that

$$
\mathbf{v}^{1}=a\left(w_{0} *_{\mathbf{w}^{1}} \mathbf{v}^{1}\right), \quad \mathbf{v}^{1}+\mathbf{v}^{2}+a\left(w_{0} *_{\mathbf{w}^{2}} \mathbf{v}^{2}\right)=\mathbf{v} .
$$

We shall write " $\mathbf{v}^{1}+\mathbf{v}^{2}=\mathbf{v}$ " if the condition (50) is satisfied. In general, we can consider a 1-parameter subgroup in $\mathrm{G}_{\mathbf{w}}^{\sigma}$ defined by

$$
\lambda: \mathbb{C}^{\times} \rightarrow \mathrm{G}_{\mathbf{w}}^{\sigma}, t \mapsto \operatorname{id}_{W^{1}} \oplus \bigoplus_{i=2}^{m}\left(t^{\lambda_{i}} \operatorname{id}_{W^{i}} \oplus t^{-\lambda_{i}} \operatorname{id}_{W^{i,-}}\right),
$$

where the pair $\left(W^{i}, W^{i,-}\right)$ play a similar role as $\left(W^{2}, W^{3}\right)$ in (42) and $0<\lambda_{1}<$ $\lambda_{2}<\cdots<\lambda_{m}$. By applying the same argument, it gives rise to the following decomposition:

$$
\mathfrak{S}_{\zeta}(\mathbf{v}, \mathbf{w})^{\lambda\left(\mathbb{C}^{\times}\right)} \cong \coprod_{\mathbf{v}^{1}+\left(\sum_{i=2}^{m} \mathbf{v}^{i}\right) \models \mathbf{v}} \mathfrak{S}_{\zeta}\left(\mathbf{v}^{1}, \mathbf{w}^{1}\right) \times \prod_{i=2}^{m} \mathfrak{M}_{\zeta}\left(\mathbf{v}^{i}, \mathbf{w}^{i}\right),
$$


where the product is taken in the natural order. By summing up all $\mathbf{v}$, the above decomposition gives rise to the following. See N01 for a comparison.

Proposition 5.2.2. Assume that $\lambda$ is given by (51), and there is an isomorphism:

$$
\mathfrak{S}_{\zeta}(\mathbf{w})^{\lambda\left(\mathbb{C}^{\times}\right)} \cong \mathfrak{S}_{\zeta}\left(\mathbf{w}^{1}\right) \times \prod_{i=2}^{m} \mathfrak{M}_{\zeta}\left(\mathbf{w}^{i}\right), \quad \mathbf{w}=\mathbf{w}^{1}+2 \sum_{i=2}^{m} \mathbf{w}^{i}, a\left(\mathbf{w}^{i}\right)=\mathbf{w}^{i} .
$$

Now consider an arbitrary torus $T \in G_{\mathbf{w}}^{\sigma}$ and the space $\operatorname{Hom}\left(\mathbb{C}^{\times}, T\right)$ of 1parameter subgroups in $T$. We form the real form $\operatorname{Hom}\left(\mathbb{C}^{\times}, T\right) \otimes_{\mathbb{Z}} \mathbb{R}$. There are generic 1-parameter subgroups in T, i.e., those $\lambda$ such that $\mathfrak{S}_{\zeta}(\mathbf{v}, \mathbf{w})^{\lambda\left(\mathbb{C}^{\times}\right)}=$ $\mathfrak{S}_{\zeta}(\mathbf{v}, \mathbf{w})^{\mathrm{T}}$. The remaining ones are called special, giving rise to larger fixedpoint subvarieties. The special 1-parameter subgroups form unions of hyperplanes, i.e., walls, in $\operatorname{Hom}\left(\mathbb{C}^{\times}, \mathrm{T}\right) \otimes_{\mathbb{Z}} \mathbb{R}$, separating generic 1-parameter subgroups into chambers, i.e., connected components of the complements of the unions of walls. From our analysis above, we see that if $T$ is a maximal torus in $G_{\mathbf{w}}^{\sigma}$, then the chamber structure can be identified with the usual Weyl chambers of type $B / C$.

5.3. Coideal structure. We shall write $\mathrm{IC}_{X}$ as the intersection cohomology complex attached to an algebraic variety $X$ (see [BBD82]). In particular, if $X=\bigsqcup_{i=1}^{n} X_{i}$ is a disjoint union of irreducible smooth varieties, then $\mathrm{IC}_{X}=\bigoplus_{i=1}^{n} \mathbb{C}_{X_{i}}\left[\operatorname{dim} X_{i}\right]$, where $\mathbb{C}_{X_{i}}$ is the constant sheaf on $X_{i}$ with coefficients in $\mathbb{C}$.

Recall from (36) that there is a proper map

$$
\pi^{\sigma}: \mathfrak{S}_{\zeta}(\mathbf{w}) \rightarrow \mathfrak{S}_{0}(\mathbf{w})
$$

So one can consider the following complex:

$$
\mathrm{P}_{\mathfrak{S}_{0}(\mathbf{w})}=\left(\pi^{\sigma}\right) ! \mathrm{IC}_{\mathfrak{S}_{\zeta}(\mathbf{w})} .
$$

Similarly, we define the complexes $\mathrm{P}_{\mathfrak{M}_{0}(\mathbf{w})}$ and $\mathrm{P}_{\mathfrak{S}_{0}(\mathbf{w})^{T}}$. The complexes $\mathrm{P}_{\mathfrak{S}_{0}(\mathbf{w})}$, $\mathrm{P}_{\mathfrak{M}_{0}(\mathbf{w})}$, and $\mathrm{P}_{\mathfrak{S}_{0}(\mathbf{w})^{T}}$ are semisimple perverse sheaves, since the map $\pi^{\sigma}$ is semismall by Corollary 4.4.2, Now we study the hyperbolic localization/restriction functor of Braden [B03] and Drinfeld-Gaitsgory DG14] on the level of $\sigma$-quiver varieties.

Theorem 5.3.1. There exists a canonical isomorphism

$$
\operatorname{can}_{\mathcal{C}}: \mathrm{P}_{\mathfrak{S}_{0}(\mathbf{w})^{\mathrm{T}}} \stackrel{\cong}{\longrightarrow} \kappa_{*}^{+}\left(\iota^{+}\right) ! \mathrm{P}_{\mathfrak{S}_{0}(\mathbf{w})},
$$

where $\kappa^{+}$and $\iota^{+}$are in (41) with the 1-parameter subgroup of $\mathrm{T}$ in the chamber $\mathrm{C}$.

Proof. For each $z \in \mathbb{C}$, let $\zeta_{\mathbb{C}}(z) \in \mathbb{C}^{I}$ be the element whose $i$ th component is $z$. Let $\zeta(z)=\left(\xi, \zeta_{\mathbb{C}}(z)\right)$, where $\xi \in \mathbb{C}^{I}$ is the element whose $i$ th component is 1 . We consider

$$
\mathfrak{S}^{\mathbf{h}}(\mathbf{v}, \mathbf{w})=\bigsqcup_{z \in \mathbb{C}} \mathfrak{S}_{\zeta(z)}(\mathbf{v}, \mathbf{w}) \quad \text { and } \quad \mathfrak{S}_{1}^{\mathbf{h}}(\mathbf{v}, \mathbf{w})=\bigsqcup_{z \in \mathbb{C}} \mathfrak{S}_{\zeta(z), 1}(\mathbf{w})
$$

where $\mathfrak{S}_{\zeta(z), 1}(\mathbf{v}, \mathbf{w})$ is the $\mathfrak{S}_{1}(\mathbf{v}, \mathbf{w})$ with $\zeta(z)$ emphasized. Similarly, one can consider $\mathfrak{M}^{\mathbf{*}}(\mathbf{v}, \mathbf{w})$ and $\mathfrak{M}_{1}^{\boldsymbol{\alpha}}(\mathbf{v}, \mathbf{w})$. These are algebraic varieties defined in a similar way as $\mathfrak{M}_{\zeta}(\mathbf{v}, \mathbf{w})$ and $\mathfrak{M}_{1}(\mathbf{v}, \mathbf{w})$, and so are $\mathfrak{S}^{\boldsymbol{\alpha}}(\mathbf{v}, \mathbf{w})$ and $\mathfrak{S}_{1}^{\boldsymbol{\phi}}(\mathbf{v}, \mathbf{w})$ as fixed-point subvarieties of automorphisms on the former algebraic varieties. Similarly, there is 
a proper morphism over $\mathbb{C}$ :

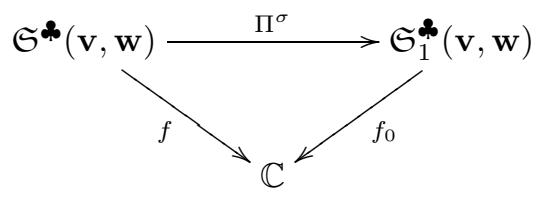

where the morphisms to $\mathbb{C}$ are defined by sending a point in $\mathfrak{S}_{\zeta(z)}(\mathbf{v}, \mathbf{w})$ and $\mathfrak{S}_{\zeta(z), 1}(\mathbf{v}, \mathbf{w})$ to $z$. The $\Pi^{\sigma}$ is a 1-parameter deformation of $\pi^{\sigma}$. In particular, the fiber of $\Pi^{\sigma}$ over $0 \in \mathbb{C}$ is exactly $\pi^{\sigma}$. Moreover, the fiber over $\mathbb{C}-\{0\}$ is an isomorphism

$$
\mathfrak{S}^{\mathbf{\alpha}}(\mathbf{v}, \mathbf{w}) \backslash \mathfrak{S}_{\zeta}(\mathbf{v}, \mathbf{w}) \cong \mathfrak{S}_{1}^{\mathbf{k}}(\mathbf{v}, \mathbf{w}) \backslash \mathfrak{S}_{1}(\mathbf{v}, \mathbf{w}) .
$$

Now we apply the argument in [N16, 5.4]. By (56), there is a canonical isomorphism

$$
\left.\psi_{f_{0}}[-1] \mathrm{IC}_{\mathfrak{S}_{1}^{*}(\mathbf{v}, \mathbf{w})}\right|_{\mathfrak{S}_{1}^{*}(\mathbf{v}, \mathbf{w}) \backslash \mathfrak{S}_{1}(\mathbf{v}, \mathbf{w})} \cong \pi_{!}^{\sigma} \operatorname{IC}_{\mathfrak{S}(\mathbf{v}, \mathbf{w})},
$$

where $\psi_{f_{0}}$ is the nearby cycle functor with respect to $f_{0}$. Similarly, there is an isomorphism

$$
\left.\psi_{f_{0}^{\mathrm{T}}}[-1] \mathrm{IC}_{\mathfrak{S}_{1}^{\mathbf{k}}(\mathbf{v}, \mathbf{w})^{\mathrm{T}}}\right|_{\mathfrak{S}_{1}^{\mathbf{*}}(\mathbf{v}, \mathbf{w})^{\mathrm{T}} \backslash \mathfrak{S}_{1}(\mathbf{v}, \mathbf{w})^{\mathrm{T}}} \cong \pi_{!}^{\sigma, \mathrm{T}} \mathrm{IC}_{\mathfrak{S}(\mathbf{v}, \mathbf{w})^{\mathrm{T}}},
$$

where $f_{0}^{\mathrm{T}}$ is an analogue of $f_{0}$ in (55) and $\pi^{\sigma, \mathrm{T}}$ is the restriction of $\pi^{\sigma}$ to its T-fixed point part. By (56) and the relative symplectic form on $\mathfrak{M}^{\mathbf{*}}(\mathbf{v}, \mathbf{w})$ induced from (5), it yields a canonical isomorphism

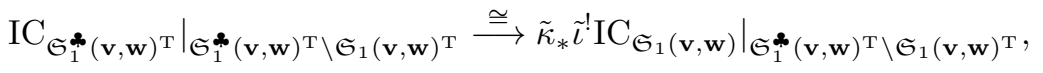

where $\tilde{\kappa}$ and $\tilde{\iota}$ are the counterparts of $\kappa^{+}$and $\iota^{+}$, respectively, on $\mathfrak{S}_{0}^{\mathbf{k}}(\mathbf{v}, \mathbf{w})$. Therefore, there is a canonical isomorphism

$$
\begin{aligned}
& \left.\pi_{!}^{\sigma, \mathrm{T}} \mathrm{IC}_{\mathfrak{S}(\mathbf{v}, \mathbf{w})^{\mathrm{T}}} \stackrel{\sqrt[58]{\cong}}{\cong} \pi_{!}^{\sigma, \mathrm{T}} \psi_{f^{\mathrm{T}}}[-1] \mathrm{IC}_{\mathfrak{S} *(\mathbf{v}, \mathbf{w})^{\mathrm{T}}}\right|_{\mathfrak{S} *(\mathbf{v}, \mathbf{w})^{\mathrm{T}} \backslash \mathfrak{S}(\mathbf{v}, \mathbf{w})^{\mathrm{T}}} \\
& \left.\stackrel{(\star)}{\cong} \psi_{f_{0}^{\mathrm{T}}}[-1] \Pi_{!}^{\sigma, \mathrm{T}} \mathrm{IC}_{\mathfrak{S}} *(\mathbf{v}, \mathbf{w})^{\mathrm{T}}\right|_{\mathfrak{S}} *(\mathbf{v}, \mathbf{w})^{\mathrm{T}} \backslash \mathfrak{S}(\mathbf{v}, \mathbf{w})^{\mathrm{T}} \\
& \left.\stackrel{(\dagger)}{\cong} \psi_{f_{0}^{\mathrm{T}}}[-1] \mathrm{IC}_{\mathfrak{S}_{1}^{*}(\mathbf{v}, \mathbf{w})^{\mathrm{T}}}\right|_{\mathfrak{S}_{1}^{*}(\mathbf{v}, \mathbf{w})^{\mathrm{T}} \backslash \mathfrak{S}_{1}(\mathbf{v}, \mathbf{w})^{\mathrm{T}}} \\
& \left.\stackrel{(59)}{\cong} \psi_{f_{0}^{\mathrm{T}}}[-1] \tilde{\kappa}_{*} \iota^{\imath} \mathrm{IC}_{\mathfrak{S}_{1}^{*}(\mathbf{v}, \mathbf{w})}\right|_{\mathfrak{S}_{1}^{*}(\mathbf{v}, \mathbf{w})^{\mathrm{T}} \backslash \mathfrak{S}_{1}(\mathbf{v}, \mathbf{w})^{\mathrm{T}}} \\
& \left.\stackrel{(b)}{\cong}\left(\kappa^{+}\right)_{*}\left(\iota^{+}\right) ! \psi_{f_{0}}[-1] \operatorname{IC}_{\mathfrak{S}_{1}^{*}(\mathbf{v}, \mathbf{w})}\right|_{\mathfrak{S}_{1}^{*}(\mathbf{v}, \mathbf{w}) \backslash \mathfrak{S}_{1}(\mathbf{v}, \mathbf{w})} \\
& \stackrel{\sqrt[577]{\cong}}{\cong}\left(\kappa^{+}\right)_{*}\left(\iota^{+}\right) ! \pi_{!}^{\sigma} \mathrm{IC}_{\mathfrak{S}(\mathbf{v}, \mathbf{w})},
\end{aligned}
$$

where $(\star)$ is due to the fact that a nearby cycle functor commutes with proper maps, $(\dagger)$ is due to the fact that $\pi^{\mathrm{T}}$ is an isomorphism when it restricts to $\mathfrak{S}^{\mathbf{d}}(\mathbf{v}, \mathbf{w})^{\mathrm{T}} \backslash$ $\mathfrak{S}(\mathbf{v}, \mathbf{w})^{\mathrm{T}}$, and $(b)$ is due to the fact that a nearby cycle functor commutes with hyperbolic restrictions. The theorem follows by summing the above (60) over all $\mathbf{v}$.

Remark 5.3.2.

(1) We refer the reader to [N13, 3(iv)] for the subtleties in choosing an isomorphism in (54). 
(2) Since a nearby cycle functor, shifted by [-1], sends perverse sheaves to perverse sheaves, the isomorphism (57) implies that the complex $\pi_{*} \mathrm{IC}_{\mathfrak{S}(\mathbf{v}, \mathbf{w})}$ is a semisimple perverse sheaf. This in turn implies that the map $\pi^{\sigma}$ is semismall onto its image (see Corollary 4.4.2).

By Proposition 5.2.2, we see that there is a canonical isomorphism

$$
\mathrm{P}_{\mathfrak{S}_{0}(\mathbf{w})^{\mathrm{T}}} \cong \bigoplus_{\mathbf{v}^{2} \models \mathbf{v}} \pi_{!}^{\sigma} \mathrm{IC}_{\mathfrak{S}_{\zeta}\left(\mathbf{w}^{1}\right)} \otimes \pi_{!} \mathrm{IC}_{\mathfrak{M}_{\zeta}\left(\mathbf{w}^{2}\right)}
$$

Thus the complex $\mathrm{P}_{\mathfrak{S}_{0}(\mathbf{w})^{\mathrm{T}}}$ is a direct summand of the complex $\mathrm{P}_{\mathfrak{S}_{0}\left(\mathbf{w}^{1}\right)} \otimes \mathrm{P}_{\mathfrak{M}_{0}\left(\mathbf{w}^{2}\right)}$. So the restriction functor $\kappa_{*}^{+}\left(\iota^{+}\right)$! induces an algebra homomorphism

$$
\Delta_{\mathbf{w}^{1}, \mathbf{w}^{2}}^{\sigma}: \operatorname{End}\left(\mathrm{P}_{\mathfrak{S}_{0}(\mathbf{w})}\right) \rightarrow \operatorname{End}\left(\mathrm{P}_{\mathfrak{S}_{0}\left(\mathbf{w}^{1}\right)}\right) \otimes \operatorname{End}\left(\mathrm{P}_{\mathfrak{M}_{0}\left(\mathbf{w}^{2}\right)}\right),
$$

where $\mathbf{w}^{1}+2 \mathbf{w}^{2}=\mathbf{w}$ and $a \mathbf{w}^{i}=\mathbf{w}^{i}$ for $i=1,2$. (Here the endomorphisms are taken inside abelian categories of perverse sheaves.)

Now we consider the following Steinberg-like varieties:

$$
\mathfrak{Y}_{\zeta}(\mathbf{w})=\bigsqcup_{\mathbf{v}^{1}, \mathbf{v}^{2}} \mathfrak{Y}_{\zeta}\left(\mathbf{v}^{1}, \mathbf{v}^{2}, \mathbf{w}\right), \quad \mathfrak{Y}_{\zeta}\left(\mathbf{v}^{1}, \mathbf{v}^{2}, \mathbf{w}\right)=\mathfrak{S}_{\zeta}\left(\mathbf{v}^{1}, \mathbf{w}\right) \times_{\mathfrak{S}_{0}(\mathbf{w})} \mathfrak{S}_{\zeta}\left(\mathbf{v}^{2}, \mathbf{w}\right)
$$

Similarly, the notation $\mathfrak{Z}_{\zeta}(\mathbf{w})$ is defined with respect to Nakajima varieties $\mathfrak{M}_{\zeta}(\mathbf{v}, \mathbf{w})$. Let $\mathrm{H}_{t o p}(X)$ denote the top Borel-Moore homology of $X$; see CG. From CG, 8.9.7], there is an algebra isomorphism

$$
\operatorname{End}\left(\mathrm{P}_{\mathfrak{S}_{0}(\mathbf{w})}\right) \cong \mathrm{H}_{\text {top }}\left(\mathfrak{Y}_{\zeta}(\mathbf{w})\right) \text { and } \operatorname{End}\left(\mathrm{P}_{\mathfrak{M}_{0}(\mathbf{w})}\right) \cong \mathrm{H}_{t o p}\left(\mathfrak{Z}_{\zeta}(\mathbf{w})\right)
$$

In terms of top Borel-Moore homology, the algebra homomorphism in (61) becomes the following algebra homomorphism, denoted by the same notation:

$\Delta_{\mathbf{w}^{1}, \mathbf{w}^{2}}^{\sigma}: \mathrm{H}_{\text {top }}\left(\mathfrak{Y}_{\zeta}(\mathbf{w})\right) \rightarrow \mathrm{H}_{\text {top }}\left(\mathfrak{Y}_{\zeta}\left(\mathbf{w}^{1}\right)\right) \otimes \mathrm{H}_{\text {top }}\left(\mathfrak{Z}_{\zeta}\left(\mathbf{w}^{2}\right)\right)$, if $\mathbf{w}^{1}+2 \mathbf{w}^{2}=\mathbf{w}, a \mathbf{w}^{i}=\mathbf{w}^{i}$.

In the same vein, we have an algebra homomorphism:

$$
\Delta_{\mathbf{w}^{1}, \mathbf{w}^{2}}: \mathrm{H}_{\text {top }}\left(\mathfrak{Z}_{\zeta}(\mathbf{w})\right) \rightarrow \mathrm{H}_{\text {top }}\left(\mathfrak{Z}_{\zeta}\left(\mathbf{w}^{1}\right)\right) \otimes \mathrm{H}_{\text {top }}\left(\mathfrak{Z}_{\zeta}\left(\mathbf{w}^{2}\right)\right), \quad \text { if } \mathbf{w}^{1}+\mathbf{w}^{2}=\mathbf{w} .
$$

By the canonical choice of isomorphism in Theorem 5.3.1 we obtain the following proposition.

Proposition 5.3.3. The algebra homomorphism $\Delta_{\mathbf{w}^{1}, \mathbf{w}^{2}}^{\sigma}$ satisfies the coassociativity, that is,

$$
\left(\Delta_{\mathbf{w}^{1}, \mathbf{w}^{2}}^{\sigma} \otimes 1\right) \circ \Delta_{\mathbf{w}^{1}+\mathbf{w}^{2}+\mathbf{w}^{3}, \mathbf{w}^{3}}^{\sigma}=\left(1 \otimes \Delta_{\mathbf{w}^{2}, \mathbf{w}^{3}}\right) \circ \Delta_{\mathbf{w}^{1}, \mathbf{w}^{2}+\mathbf{w}^{3}}^{\sigma},
$$

for all $\mathbf{w}^{1}+2\left(\mathbf{w}^{2}+\mathbf{w}^{3}\right)=\mathbf{w}$ and $a \mathbf{w}^{i}=\mathbf{w}^{i}$ for $i=1,2,3$.

To $\mathbf{w}$, we define $\mathbf{r}_{\mathbf{w}}$ by $\left(\mathbf{r}_{\mathbf{w}}\right)_{i}=\frac{\mathbf{w}_{i}}{2}-\frac{1-(-1)^{\mathbf{w}_{i}}}{4}$, that is, $\left(\mathbf{r}_{\mathbf{w}}\right)_{i}$ is the rank of the $i$ th isometry group with respect to the $i$ th $\delta_{\mathbf{w}, i}$-form. Let $\mathbf{s}_{\mathbf{w}}=\mathbf{w}-2 \mathbf{r}_{\mathbf{w}}$. If $\mathfrak{S}_{\zeta}\left(\mathbf{s}_{\mathbf{w}}\right)=$ $\{\mathrm{pt}\}$, then the coproduct (63) becomes the following algebra homomorphism:

$$
\jmath: \mathrm{H}_{t o p}\left(\mathfrak{Y}_{\zeta}(\mathbf{w})\right) \rightarrow \mathrm{H}_{t o p}\left(\mathfrak{Z}_{\zeta}\left(\mathbf{r}_{\mathbf{w}}\right)\right), \quad \text { if } \mathfrak{S}_{\zeta}\left(\mathbf{s}_{\mathbf{w}}\right)=\{\mathrm{pt}\} .
$$

Recall that $\Gamma$ is a Dynkin diagram and $w_{0}$ is the longest element in the associated Weyl group. There is an involution $\theta$ on $I$ such that $w_{0}\left(\alpha_{i}\right)=-\alpha_{\theta(i)}$, where $\alpha_{i}$ is the $i$ th simple root of $\Gamma$. Let $\mathfrak{g}_{\Gamma}$ be the simple Lie algebra associated to $\Gamma$ with Chevalley generators $\left\{e_{i}, f_{i}, h_{i} \mid i \in I\right\}$. Then the assignment $e_{i} \mapsto f_{a \theta(i)}, f_{i} \mapsto e_{a \theta(i)}$, and $h_{i} \mapsto-h_{a \theta(i)}$ for all $i \in I$ defines an involution, denoted by $\sigma$, on $\mathfrak{g}_{\Gamma}$. It is known 
TABLE 1

\begin{tabular}{|l|l|l|}
\hline$(\Gamma,|a|)$ & $\mathfrak{g}^{\sigma} \equiv \mathfrak{k}$ & Satake type \\
\hline$\left(\mathrm{A}_{\ell}, 1\right): \ell=2 p$ & $\mathfrak{s l}_{p} \oplus \mathfrak{g l}_{p+1}$ & AIII \\
\hline$\left(\mathrm{A}_{\ell}, 1\right): \ell=2 p-1$ & $\mathfrak{s l}_{p} \oplus \mathfrak{g l}_{p}$ & AIII \\
\hline$\left(\mathrm{A}_{\ell}, 2\right)$ & $\mathfrak{s o}_{\ell+1}$ & AI \\
\hline$\left(\mathrm{D}_{\ell}, 1\right): \ell$ odd & $\mathfrak{s o}_{\ell-1} \oplus \mathfrak{s o}_{\ell+1}$ & DI \\
\hline$\left(\mathrm{D}_{\ell}, 1\right): \ell$ even & $\mathfrak{s o}_{\ell} \oplus \mathfrak{s o}_{\ell}$ & DI \\
\hline$\left(\mathrm{D}_{\ell}, 2\right): \ell$ odd & $\mathfrak{s o}_{\ell} \oplus \mathfrak{s o}_{\ell}$ & DI \\
\hline$\left(\mathrm{D}_{\ell}, 2\right): \ell$ even & $\mathfrak{s o}_{\ell-1} \oplus \mathfrak{s o}_{\ell+1}$ & DI \\
\hline$\left(\mathrm{E}_{6}, 1\right)$ & $\mathfrak{s l}_{2} \oplus \mathfrak{s l}_{6}$ & EII \\
\hline$\left(\mathrm{E}_{6}, 2\right)$ & $\mathfrak{s p}_{4}$ & EI \\
\hline$\left(\mathrm{E}_{7}, 1\right)$ & $\mathfrak{s l}_{8}$ & EV \\
\hline$\left(\mathrm{E}_{8}, 1\right)$ & $\mathfrak{s o}_{16}$ & EVIII \\
\hline
\end{tabular}

that the fixed-point Lie subalgebra $\mathfrak{g}_{\Gamma}^{\sigma}$ is generated by $e_{i}+f_{a \theta(i)}$ and $h_{i}-h_{a \theta(i)}$ for all $i \in I$. The algebra $\mathfrak{g}_{\Gamma}^{\sigma}$ is usually denoted by $\mathfrak{k}$ in the introduction. The pair $\left(\mathfrak{g}_{\Gamma}, \mathfrak{g}_{\Gamma}^{\sigma}\right)$ then forms a so-called symmetric pair. The Lie algebra $\mathfrak{g}_{\Gamma}^{\sigma}$ is classified by the Satake diagrams without black vertices (i.e., $X=\varnothing$ in [K14]). Specifically, they are listed in Table 1.

Let $U\left(\mathfrak{g}_{\Gamma}^{\sigma}\right)$ be the universal enveloping algebra of $\mathfrak{g}_{\Gamma}^{\sigma}$. With the coassociativity and (66) in hand, we make the following conjecture.

Conjecture 5.3.4. There is a nontrivial algebra homomorphism

$$
U\left(\mathfrak{g}_{\Gamma}^{\sigma}\right) \rightarrow \mathrm{H}_{\text {top }}\left(\mathfrak{Y}_{\zeta}(\mathbf{w})\right)
$$

When the Dynkin diagram $\Gamma$ is of type $A$ and $a=1$, this conjecture can be shown by the results in BKLW] and an argument similar to BG99.

5.4. The stable map Stabe. Recall that $\mathfrak{S}_{\zeta}(\mathbf{w})$ has a $\mathbb{C}^{\times}$-action by scaling. Since the maps $\pi^{\sigma}$ and $\pi^{\sigma, \mathrm{T}}$ are $\mathbb{T} \equiv \mathrm{T} \times \mathbb{C}^{\times}$-equivariant, the isomorphism can can $_{\mathcal{C}}$ in (54) also holds in the derived category of $\mathbb{T}$-equivariant $\mathbb{C}$-constructible sheaves. This $\mathbb{T}$-equivariant version of the canonical isomorphism (54) is the same as the one given by Maulik-Okounkov's stable envelope [MO12, as explained in N16] (the statement after Corollary 5.4.2 therein). With the help of cane in (54), one obtains the stable map on the torus-equivariant cohomologies:

$$
\operatorname{Stab}_{\mathcal{C}}: \mathrm{H}_{\mathbb{T}}^{[*]}\left(\mathfrak{S}_{\zeta}(\mathbf{w})^{\mathrm{T}}\right) \rightarrow \mathrm{H}_{\mathbb{T}}^{[*]}\left(\mathfrak{S}_{\zeta}(\mathbf{w})\right)
$$

where $[*]$ is the shifted degree defined by $\mathrm{H}_{\mathbb{T}}^{[*]}(?)=\mathrm{H}_{\mathbb{T}}^{*+\operatorname{dim}}(?)$. This sheaf-theoretic definition of Stabe is formal and contained in [N16]. For the convenience of the reader, we reproduce it here. For simplicity, we write $\mathfrak{X} \equiv \mathfrak{S}_{\zeta}(\mathbf{w})$ and $\mathfrak{X}_{0} \equiv \mathfrak{S}_{0}(\mathbf{w})$ in this process. There are canonical isomorphisms/identifications:

$$
\mathrm{H}_{\mathbb{T}}^{[*]}\left(\mathfrak{X}^{\mathrm{T}}\right) \cong \operatorname{Ext}_{\mathbb{T}}^{*}\left(\mathbb{C}_{\mathfrak{X}^{\mathrm{T}}}, \mathrm{IC}_{\mathfrak{X}^{\mathrm{T}}}\right) \cong \operatorname{Ext}_{\mathbb{T}}^{*}\left(\left(\pi^{\sigma, \mathrm{T}}\right)^{*} \mathbb{C}_{\mathfrak{X}_{0}^{\mathrm{T}}}, \mathrm{IC}_{\mathfrak{X}^{\mathrm{T}}}\right) \cong \operatorname{Ext}_{\mathbb{T}}^{*}\left(\mathbb{C}_{\mathfrak{X}_{0}^{\mathrm{T}}},\left(\pi^{\sigma, \mathrm{T}}\right)_{*} \mathrm{IC}_{\mathfrak{X}^{\mathrm{T}}}\right)
$$


On the other hand, there are canonical isomorphisms

$$
\begin{aligned}
& \operatorname{Ext}_{\mathbb{T}}^{*} *\left(\mathbb{C}_{\mathfrak{X}_{0}^{\mathrm{T}}}, \kappa_{*}^{+}\left(\iota^{+}\right) ! \pi_{*}^{\sigma} \mathrm{IC}_{\mathfrak{X}}\right) \cong \operatorname{Ext}_{\mathbb{T}}^{*}\left(\mathbb{C}_{\mathfrak{X}_{0}^{+\mathbb{C} \times}},\left(\iota^{+}\right) ! \pi_{*}^{\sigma} \mathrm{IC}_{\mathfrak{X}}\right) \\
& \cong \operatorname{Ext}_{\mathbb{T}}^{*}\left(\mathbb{C}_{\mathfrak{X}_{0}^{+\mathbb{C}^{\times}}},\left(\tilde{\pi}^{\sigma}\right)_{*}(\tilde{\iota}) ! \mathrm{IC}_{\mathfrak{X}}\right) \\
& \cong \operatorname{Ext}_{\mathbb{T}}^{*}\left(\mathbb{C}_{\mathfrak{X}+\mathbb{C} \times},(\tilde{\iota})^{!} \mathrm{IC}_{\mathfrak{X}}\right) \\
& \cong \operatorname{Ext}_{\mathbb{T}}^{*}\left(\mathbb{C}_{\mathfrak{X}},(\tilde{\iota})_{!}(\tilde{\iota})^{!} \mathrm{IC}_{\mathfrak{X}}\right),
\end{aligned}
$$

where the 1-parameter subgroup $\mathbb{C}^{\times}$is chosen from the chamber $\mathcal{C}, \tilde{\pi}^{\sigma}$ and $\tilde{\iota}$ are given in the following cartesian diagram:

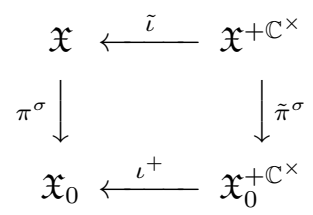

Note that there is an adjunction adj : $(\tilde{\iota})_{!}(\tilde{\iota})^{!} \rightarrow \mathrm{id}$, which induces a morphism

$$
\operatorname{adj}: \operatorname{Ext}_{\mathbb{T}}^{*}\left(\mathbb{C}_{\mathfrak{X}},(\tilde{\iota}) !(\tilde{\iota}) ! \mathrm{IC}_{\mathfrak{X}}\right) \rightarrow \operatorname{Ext}_{\mathbb{T}}^{*}\left(\mathbb{C}_{\mathfrak{X}}, \mathrm{IC}_{\mathfrak{X}}\right)=\mathrm{H}_{\mathbb{T}}^{[*]}(\mathfrak{X}) \text {. }
$$

The stable map is thus defined to be the following composition:

$$
\begin{aligned}
\mathrm{H}_{\mathbb{T}}^{[*]}\left(\mathfrak{X}^{\mathrm{T}}\right) \stackrel{(68)}{=} \operatorname{Ext}_{\mathbb{T}}^{*}\left(\mathbb{C}_{\mathfrak{X}},(\tilde{\iota}) !(\tilde{\iota})^{!} \mathrm{IC}_{\mathfrak{X}}\right) & \stackrel{\operatorname{can} \rho}{\longrightarrow} \operatorname{Ext}_{\mathbb{T}}^{*} *\left(\mathbb{C}_{\mathfrak{X}_{0}^{\mathrm{T}}}, \kappa_{*}^{+}\left(\iota^{+}\right)^{!} \pi_{*}^{\sigma} \mathrm{IC}_{\mathfrak{X}}\right) \\
& \stackrel{(69)}{=} \operatorname{Ext}_{\mathbb{T}}^{*}\left(\mathbb{C}_{\mathfrak{X}},(\tilde{\iota}) !(\tilde{\iota})^{!} \mathrm{IC}_{\mathfrak{X}}\right) \stackrel{\text { adj }}{\longrightarrow} \mathrm{H}_{\mathbb{T}}^{[*]}(\mathfrak{X}) .
\end{aligned}
$$

So, modulo the canonical identifications in (68) and (69), we have Stabe $=$ adj $\circ$ cane.

5.5. Universal $\mathcal{K}$-matrix. Let $\mathbb{C}[\operatorname{Lie}(\mathbb{T})]$ be the coordinate ring of the Lie algebra Lie $(\mathbb{T})$, as an affine space. Let $\mathbf{F}_{\mathrm{T}}$ be its rational field. The cohomologies $\mathrm{H}_{\mathbb{T}}^{[*]}\left(\mathfrak{S}_{\zeta}(\mathbf{w})^{\mathrm{T}}\right)$ and $\mathrm{H}_{\mathbb{T}}^{[*]}\left(\mathfrak{S}_{\zeta}(\mathbf{w})\right)$ are $\mathbb{C}[\operatorname{Lie}(\mathbb{T})]$-modules $\left(\right.$ since $\mathrm{H}_{\mathbb{T}}^{*}(\{\mathrm{pt}\})=\mathbb{C}[\operatorname{Lie}(\mathbb{T})]$ ) and the map Stab is compatible with the $\mathbb{C}[\operatorname{Lie}(\mathbb{T})]$-module structures. It is known that after a change of coefficients from $\mathbb{C}[\operatorname{Lie}(\mathbb{T})]$ to $\mathbf{F}_{\mathrm{T}}$, the stable map is invertible. Following Maulik-Okounkov MO12, the $\mathcal{K}$-matrix is defined by

$$
\mathcal{K}_{\mathcal{C}^{\prime}, \mathcal{C}}=\operatorname{Stab}_{\mathcal{C}^{\prime}}^{-1} \circ \operatorname{Stab}_{\mathcal{C}} \in \operatorname{End}_{\mathbb{C}[\operatorname{Lie}(\mathbb{T})]}\left(\mathrm{H}_{\mathbb{T}}^{*}\left(\mathfrak{S}_{\zeta}(\mathbf{w})^{\mathrm{T}}\right)\right) \otimes_{\mathbb{C}[\operatorname{Lie}(\mathbb{T})]} \mathbf{F}_{\mathrm{T}}
$$

Clearly, one has

$$
\mathcal{K}_{\mathcal{C}^{\prime \prime}, \mathcal{C}^{\prime}} \mathcal{K}_{\mathcal{C}^{\prime}, \mathcal{C}}=\mathcal{K}_{\mathcal{C}^{\prime \prime}, \mathcal{e}} \quad \text { and } \quad \mathcal{K}_{\mathcal{C}, \mathcal{e}}=1 .
$$

If the chamber $\mathcal{C}$ is determined by the inequalities $a_{m}>\cdots>a_{1}>0$ and $\mathfrak{S}(\mathbf{w})^{\mathrm{T}} \cong \prod_{i=1}^{m} \mathfrak{M}\left(\mathbf{w}^{i}\right)$, we write $\mathcal{K}_{-\mathcal{e}, \mathcal{e}}$ by $\mathcal{K}_{\underline{\mathbf{w}}}(\underline{a})$, where $\underline{\mathbf{w}}=\left(\mathbf{w}_{1}, \cdots, \mathbf{w}_{m}\right)$ and $\underline{a}=\left(a_{1}, \cdots, a_{m}\right)$. For $\underline{\mathbf{w}}=\left(\mathbf{w}^{1}\right)$ and $\underline{a}=\left(a_{1}\right)$, we write $\mathcal{K}_{\mathbf{w}^{1}}\left(a_{1}\right)$ for $\mathcal{K}_{\underline{\mathbf{w}}}(\underline{a})$. Let us list an example of the $\mathcal{K}$-matrix.

Example 5.5.1. Let $\Gamma=A_{1}$ and $\mathbf{w}=2$ with $\delta_{\mathbf{w}}=-1$ so that $\mathfrak{S}_{\zeta}(\mathbf{w})=T^{*} \mathcal{B}^{\mathfrak{s p}_{2}} \cong$ $T^{*} \mathbb{P}^{1}$ is the cotangent bundle of the complete flag variety of $\mathrm{Sp}_{2} \cong \mathrm{SL}_{2}$. So by [MO12, 4.1.2], we have

$$
\mathcal{K}_{1}(a)=\frac{1-\frac{\hbar}{a}\left[\begin{array}{ll}
0 & 1 \\
1 & 0
\end{array}\right]}{1-\frac{\hbar}{a}} \in \operatorname{End}\left(\mathbb{C}^{2}(\hbar, a)\right) .
$$




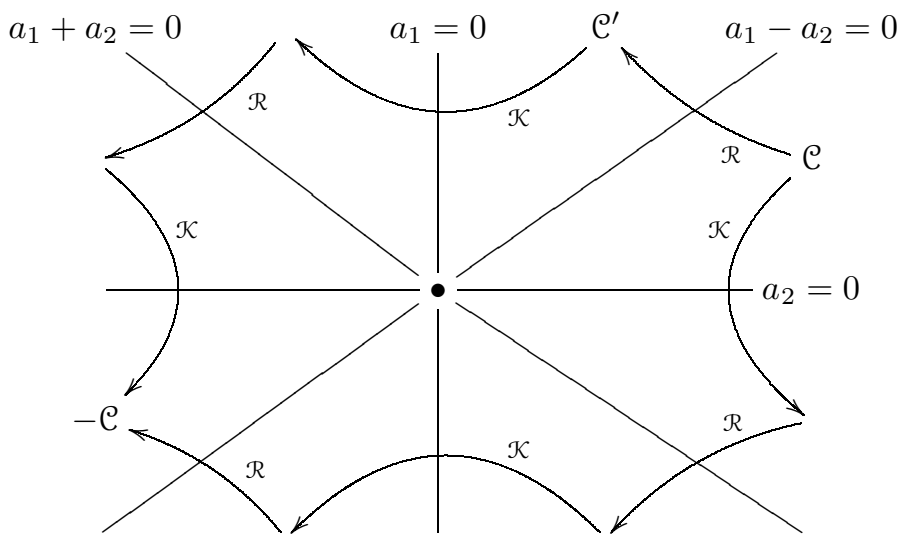

FIgURE 1. Reflection equation

Since $\mathcal{K}_{\mathfrak{C}, \mathcal{e}}=1$, the identity in (73) yields the following proposition.

Proposition 5.5.2. The $\mathcal{K}$-matrix is unitary, i.e., $\mathcal{K}_{\mathbf{w}}(-a)=\mathcal{K}_{\mathbf{w}}(a)^{-1}$.

Suppose that $\mathrm{T}$ is a two-dimensional torus in $\mathrm{G}_{\mathbf{w}}^{\sigma}$. A typical 1-parameter subgroup in $\mathrm{T}$ is given similar to (51) for various $\left(a_{1}, a_{2}\right) \in \mathbb{Z}^{2}$ :

$$
\lambda_{a_{1}, a_{2}}: \mathbb{C}^{\times} \rightarrow \mathrm{G}_{\mathbf{w}}^{\sigma}, t \mapsto \operatorname{id}_{W^{0}} \oplus\left(t^{a_{1}} \operatorname{id}_{W^{1}} \oplus t^{-a_{1}} \operatorname{id}_{W^{1,-}}\right) \oplus\left(t^{a_{2}} \mathrm{id}_{W^{2}} \oplus t^{-a_{2}} \operatorname{id}_{W^{2,-}}\right) .
$$

The real form of $\operatorname{Lie}(\mathrm{T})$ is thus a plane $\mathbb{R}^{2}$ whose walls are the lines $a_{1}=0, a_{2}=0$, $a_{1}-a_{2}=0$, and $a_{1}+a_{2}=0$. In particular, there are 8 chambers in Lie(T), which is exactly the Weyl chambers of type $B_{2} / C_{2}$ (see Figure1). Let $\mathcal{R}_{\mathcal{C}^{\prime}, \mathcal{e}}$ denote Maulik-Okounkov's $\mathcal{R}$-matrix on the torus equivariant cohomology of Nakajima variety. Under this setting, the $\mathcal{K}$-matrix satisfies the reflection equation, instead of the Yang-Baxter equation for the $\mathcal{R}$-matrix.

Proposition 5.5.3. The $\mathcal{K}$-matrix satisfies the following reflection equation:

$$
\mathcal{K}_{\mathbf{w}^{2}}\left(a_{2}\right) \mathcal{R}\left(a_{1}+a_{2}\right) \mathcal{K}_{\mathbf{w}^{1}}\left(a_{1}\right) \mathcal{R}\left(a_{1}-a_{2}\right)=\mathcal{R}\left(a_{1}-a_{2}\right) \mathcal{K}_{\mathbf{w}^{1}}\left(a_{1}\right) \mathcal{R}\left(a_{1}+a_{2}\right) \mathcal{K}_{\mathbf{w}^{2}}\left(a_{2}\right),
$$

where the $\mathcal{K}_{\mathbf{w}^{i}}\left(a_{i}\right)$ 's are understood as $\mathcal{K}_{\mathbf{w}^{1}}\left(a_{1}\right) \otimes 1$ and $1 \otimes \mathcal{K}_{\mathbf{w}^{2}}\left(a_{2}\right)$, respectively.

Proof. If the $\mathcal{R}$ 's in the equation are replaced by the $\mathcal{K}$ 's, then this holds because

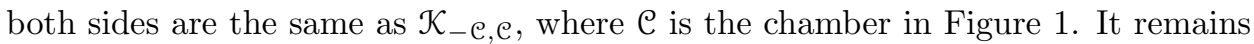
to show that $\mathcal{R}\left(a_{1} \pm a_{2}\right)=\mathcal{K}\left(a_{1} \pm a_{2}\right)$. Under the setting (74), the condition $a_{1}-a_{2}=0$ defines a subtorus $\mathrm{T}^{\prime}$ in $\mathrm{T}$ so that $\mathfrak{S}_{\zeta}(\mathbf{w})^{\mathrm{T}^{\prime}} \cong \mathfrak{S}_{\zeta}\left(\mathbf{w}^{0}\right) \times \mathfrak{M}_{\zeta}\left(\mathbf{w}^{1}+\mathbf{w}^{2}\right)$ by Proposition [5.2.1. By general properties of $\mathcal{K} / \mathcal{R}$-matrices, the $\mathcal{K}$-matrix $\mathcal{K}_{\mathcal{C}^{\prime}, \mathcal{C}}$ of crossing the wall $a_{1}-a_{2}=0$ in Figure 1 is the same as the $\mathcal{K}$-matrix for the torus $\mathrm{T} / \mathrm{T}^{\prime}$ on $\mathfrak{S}_{\zeta}(\mathbf{w})^{\mathrm{T}^{\prime}}$. Note that $\mathrm{T} / \mathrm{T}^{\prime}$ acts trivially on the component $\mathfrak{S}_{\zeta}\left(\mathbf{w}^{0}\right)$, so the latter $\mathcal{K}$-matrix is Maulik-Okounkov's original $\mathcal{R}$-matrix $\mathcal{R}\left(a_{1}-a_{2}\right)$ on quiver varieties. This shows that $\mathcal{K}\left(a_{1}-a_{2}\right)=\mathcal{R}\left(a_{1}-a_{2}\right)$. The other equality can be obtained by the same argument. The proposition is thus proved. 
In general, the $\mathcal{K}$-matrix $\mathcal{K}_{\underline{\mathbf{w}}}(\underline{a})$ can be obtained from the $\mathcal{K}_{\mathbf{w}^{i}}\left(a_{i}\right)$ 's via the socalled fusion procedure. In particular, when $\underline{\mathbf{w}}$ contains two components, it reads as follows.

Proposition 5.5.4. One has

$$
\begin{aligned}
\mathcal{K}_{\mathbf{w}^{1}, \mathbf{w}^{2}}\left(a_{1}, a_{2}\right) & =\mathcal{R}\left(a_{2}-a_{1}\right) \mathcal{K}_{\mathbf{w}^{2}}\left(a_{2}\right) \mathcal{R}\left(a_{1}+a_{2}\right) \mathcal{K}_{\mathbf{w}^{1}}\left(a_{1}\right) \\
& =\mathcal{K}_{\mathbf{w}^{1}}\left(a_{1}\right) \mathcal{R}\left(a_{1}+a_{2}\right) \mathcal{K}_{\mathbf{w}^{2}}\left(a_{2}\right) \mathcal{R}\left(a_{2}-a_{1}\right) .
\end{aligned}
$$

Proof. We have $\mathcal{K}_{\mathbf{w}^{1}, \mathbf{w}^{2}}=\mathcal{K}_{-\mathcal{C}^{\prime}, \mathcal{C}^{\prime}}\left(a_{1}, a_{2}\right)$, where the chamber $\mathcal{C}^{\prime}$ is given in Figure 1. The proposition follows by multiplying $\mathcal{R}\left(a_{2}-a_{1}\right)$ on both sides of Equation (75) and using the unitary property of the $\mathcal{K}$-matrix.

Remark 5.5.5. As we learnt from Weiqiang Wang, the algebraic $K$-matrix for quantum symmetric pairs of type AIII/IV first appeared in BW13. The relationship between the $\mathcal{K}$-matrix in this section and the algebraic ones in BaK16] is not clear.

5.6. Twisted Yangian via the FRT formalism. Let $y$ be Maulik-Okounkov's Yangian, which is formulated in the framework of Faddeev-Reshetikhin-Takhtajan [FRT]. In particular, the algebra $y$ is a subalgebra in the product $\prod_{\mathbf{w}, \mathrm{T}} \mathrm{H}_{\mathbb{T}}^{*}\left(\mathfrak{S}_{\zeta}(\mathbf{w})^{\mathrm{T}}\right)$ $\otimes \mathbf{F}_{\mathbb{T}}$ generated by the matrix coefficients in the $\mathcal{R}$-matrix $\mathcal{R}_{0,1}\left(a_{0}-a_{1}\right) \cdots \mathcal{R}_{0, m}\left(a_{0}-\right.$ $a_{m}$ ) with respect to $a_{0}$ (see [MO12, 6.2.6]). Let $y_{\sigma}$ be the subalgebra of $y$ generated by the matrix coefficients with respect to $a_{0}$ of the operators

$$
\mathcal{R}_{0, m}\left(a_{0}-a_{m}\right) \cdots \mathcal{R}_{0,1}\left(a_{0}-a_{1}\right) \cdot \mathcal{K}_{0}\left(a_{0}\right) \cdot \mathcal{R}_{0,1}\left(a_{0}-a_{1}\right) \cdots \mathcal{R}_{0, m}\left(a_{0}-a_{m}\right) .
$$

Note that operators of the above form satisfy the reflection equation, which can be shown by induction in the following. In light of this property, we shall call $y_{\sigma}$ a twisted Yangian.

Proposition 5.6.1. Let $\mathcal{R}_{i j}\left(a_{i}-a_{j}\right)$ be an $\mathcal{R}$-matrix at the $(i, j)$-component on the tensor $F_{0}\left(a_{0}\right) \otimes F_{1}\left(a_{1}\right) \otimes \cdots \otimes F_{m}\left(a_{m}\right)$. Let $\mathcal{K}_{0}\left(a_{0}\right)$ be a $\mathcal{K}$-matrix at the 0 component. Then the operator, say $\mathcal{S}\left(a_{0}\right)$, in (77) satisfies the reflection equation

$$
\mathcal{R}_{0,1}\left(a_{0}-b_{0}\right) \mathcal{S}_{0}\left(a_{0}\right) \mathcal{R}_{0,1}\left(a_{0}+b_{0}\right) \mathcal{S}_{1}\left(b_{0}\right)=\mathcal{S}_{1}\left(b_{0}\right) \mathcal{R}_{0,1}\left(a_{0}+b_{0}\right) \mathcal{S}_{0}\left(a_{0}\right) \mathcal{R}_{0,1}\left(a_{0}-b_{0}\right),
$$

in the tensor $F_{0}\left(a_{0}\right) \otimes F_{0}\left(b_{0}\right) \otimes F_{1}\left(a_{1}\right) \otimes \cdots \otimes F_{m}\left(a_{m}\right)$.

Proof. We shall prove the proposition by induction. When $m=1$, we shift the subindex by 1 and set $\left(a_{0}, b_{0}, a_{1}\right)=(u, v, w)$. Then we have

$$
\begin{aligned}
& \mathcal{R}_{12}(u-v) \mathcal{S}_{1}(u) \mathcal{R}_{12}(u+v) \mathcal{S}_{2}(v) \\
& =\mathcal{R}_{12}(u-v)\left(\mathcal{R}_{13}(u-w) \mathcal{K}_{1}(u) \mathcal{R}_{13}(u-w)\right) \mathcal{R}_{12}(u+v)\left(\mathcal{R}_{23}(v-w) \mathcal{K}_{2}(v) \mathcal{R}_{23}(v-w)\right) \\
& =\mathcal{R}_{12}(u-v) \mathcal{R}_{13}(u-w) \mathcal{K}_{1}(u) \mathcal{R}_{23}(v-w) \mathcal{R}_{12}(u+v) \mathcal{R}_{13}(u-w) \mathcal{K}_{2}(v) \mathcal{R}_{23}(v-w) \\
& =\mathcal{R}_{12}(u-v) \mathcal{R}_{13}(u-w) \mathcal{R}_{23}(v-w) \mathcal{K}_{1}(u) \mathcal{R}_{12}(u+v) \mathcal{K}_{2}(v) \mathcal{R}_{13}(u-w) \mathcal{R}_{23}(v-w) \\
& =\mathcal{R}_{23}(v-w) \mathcal{R}_{13}(u-w) \mathcal{R}_{12}(u-v) \mathcal{K}_{1}(u) \mathcal{R}_{12}(u+v) \mathcal{K}_{2}(v) \mathcal{R}_{13}(u-w) \mathcal{R}_{23}(v-w) \\
& =\mathcal{R}_{23}(v-w) \mathcal{R}_{13}(u-w) \mathcal{K}_{2}(v) \mathcal{R}_{12}(u+v) \mathcal{K}_{1}(u) \mathcal{R}_{12}(u-v) \mathcal{R}_{13}(u-w) \mathcal{R}_{23}(v-w) \\
& =\mathcal{R}_{23}(v-w) \mathcal{R}_{13}(u-w) \mathcal{K}_{2}(v) \mathcal{R}_{12}(u+v) \mathcal{K}_{1}(u) \mathcal{R}_{23}(v-w) \mathcal{R}_{13}(u-w) \mathcal{R}_{12}(u-v) \\
& =\mathcal{R}_{23}(v-w) \mathcal{K}_{2}(v) \mathcal{R}_{13}(u-w) \mathcal{R}_{12}(u+v) \mathcal{R}_{23}(v-w) \mathcal{K}_{1}(u) \mathcal{R}_{13}(u-w) \mathcal{R}_{12}(u-v) \\
& =\mathcal{R}_{23}(v-w) \mathcal{K}_{2}(v) \mathcal{R}_{23}(v-w) \mathcal{R}_{12}(u+v) \mathcal{R}_{13}(u-w) \mathcal{K}_{1}(u) \mathcal{R}_{13}(u-w) \mathcal{R}_{12}(u-v) \\
& =\mathcal{S}_{2}(v) \mathcal{R}_{12}(u+v) \mathcal{S}_{1}(u) \mathcal{R}_{12}(u-v),
\end{aligned}
$$


where the second equality is due to the modified Yang-Baxter equation

$$
\mathcal{R}_{13}(u-w) \mathcal{R}_{12}(u+v) \mathcal{R}_{23}(v-w)=\mathcal{R}_{23}(v-w) \mathcal{R}_{12}(u+v) \mathcal{R}_{13}(u-w),
$$

via the unitary property of $\mathcal{R}$, the third equality is due to the commutativity of $\mathcal{K}_{i}(a)$ with $\mathcal{R}_{j, k}$ if $i \neq j, k$, and the fifth one is due to the reflection equation of the $\mathcal{K}$-matrices.

In general, we write $\mathcal{S}^{(m)}(u)$ for the $\mathcal{S}$ on $F_{0}\left(a_{0}\right) \otimes \cdots \otimes F_{m}\left(a_{m}\right)$. Then we have

$$
\mathcal{S}^{(m)}(u)=\mathcal{R}_{0, m}\left(a_{0}-a_{m}\right) \mathcal{S}^{(m-1)}(u) \mathcal{R}_{0, m}\left(a_{0}-a_{m}\right) .
$$

In particular, there is the following with $\left(a_{0}, b_{0}\right)=(u, v)$ :

$$
\begin{aligned}
& \mathcal{R}_{01}(u-v) \mathcal{S}_{1}^{(m)}(u) \mathcal{R}_{01}(u+v) \mathcal{S}_{2}^{(m)}(v) \\
& =\mathcal{R}_{01}(u-v) \mathcal{R}_{0, m+1}\left(u-a_{m}\right) \mathcal{S}_{1}^{(m-1)}(u) \mathcal{R}_{0, m+1}\left(u-a_{m}\right) R_{01}(u+v) \mathcal{R}_{1, m+1}\left(v-a_{m}\right) \\
& \mathcal{S}_{2}^{(m-1)}(v) R_{1, m+1}\left(v-a_{m}\right) \\
& =\mathcal{R}_{01}(u-v) \mathcal{R}_{0, m+1}\left(u-a_{m}\right) \mathcal{R}_{1, m+1}\left(v-a_{m}\right) \mathcal{S}_{1}^{(m-1)}(u) R_{01}(u+v) \mathcal{S}_{2}^{(m-1)}(v) \\
& \mathcal{R}_{0, m+1}\left(u-a_{m}\right) R_{1, m+1}\left(v-a_{m}\right) \\
& =\mathcal{R}_{1, m+1}\left(v-a_{m}\right) \mathcal{R}_{0, m+1}\left(u-a_{m}\right) \mathcal{R}_{01}(u-v) \mathcal{S}_{1}^{(m-1)}(u) R_{01}(u+v) \mathcal{S}_{2}^{(m-1)}(v) \\
& =\mathcal{R}_{0, m+1}\left(u-a_{m}\right) R_{1, m+1}\left(v-a_{m}\right) \\
& =\mathcal{R}_{1, m+1}\left(v-a_{m}\right) \mathcal{R}_{0, m+1}\left(u-a_{m}\right) \mathcal{S}_{2}^{(m-1)}(v) R_{01}(u+v) \mathcal{S}_{1}^{(m-1)}(u) \mathcal{R}_{01}(u-v) \\
& \mathcal{R}_{0, m+1}\left(u-a_{m}\right) R_{1, m+1}\left(v-a_{m}\right) \\
& =\mathcal{R}_{1, m+1}\left(v-a_{m}\right) \mathcal{S}_{2}^{(m-1)}(v) \mathcal{R}_{0, m+1}\left(u-a_{m}\right) R_{01}(u+v) R_{1, m+1}\left(v-a_{m}\right) \mathcal{S}_{1}^{(m-1)}(u) \\
& \mathcal{R}_{0, m+1}\left(u-a_{m}\right) \mathcal{R}_{01}(u-v) \\
& =\mathcal{R}_{1, m+1}\left(v-a_{m}\right) \mathcal{S}_{2}^{(m-1)}(v) R_{1, m+1}\left(v-a_{m}\right) R_{01}(u+v) \mathcal{R}_{0, m+1}\left(u-a_{m}\right) \mathcal{S}_{1}^{(m-1)}(u) \\
& \mathcal{R}_{0, m+1}\left(u-a_{m}\right) \mathcal{R}_{01}(u-v) \\
& =\mathcal{S}_{2}^{(m)}(v) \mathcal{R}_{01}(u+v) \mathcal{S}_{1}^{(m)}(u) \mathcal{R}_{01}(u-v) .
\end{aligned}
$$

The proposition is thus proved.

From the definition, both algebras $y$ and $y_{\sigma}$ act on $\mathrm{H}_{\mathbb{T}}^{*}\left(\mathfrak{M}_{\zeta}(\mathbf{w})\right) \otimes \mathbf{F}_{\mathbb{T}}$ and their tensor products. Summing up the above analysis, it yields the following theorem.

Theorem 5.6.2. There is a $\left(y, y_{\sigma}\right)$-action on $\mathrm{H}_{\mathbb{T}}^{*}\left(\mathfrak{M}_{\zeta}(\mathbf{w})\right) \otimes \mathbf{F}_{\mathbb{T}}$ and their tensor products.

\section{Example I: Cotangent BUndles of isotropic Flag VARIETIES}

In this section, we show that a natural involution on the cotangent bundle of the $n$-step partial flag variety of type $\mathrm{A}_{n}$ is a special case of the automorphism $\sigma$. As a consequence, we show that cotangent bundles of partial flag varieties of classical type are examples of the quiver varieties $\mathfrak{S}_{\zeta}(\mathbf{v}, \mathbf{w})$. 
6.1. Notation. In this section, we assume that the graph $\Gamma$ is a Dynkin diagram of type $A_{n}$ :

$$
\mathrm{A}_{n}(n \geq 1): \quad 1 \longrightarrow 2 \underset{\longleftrightarrow}{\longleftrightarrow} n
$$

Assume further that the dimension vectors $\mathbf{v}$ and $\mathbf{w}$ of the pair of vector spaces $V$ and $W$ satisfy that $\mathbf{w}_{i}=0$ for $i \geq 2$ and $\mathbf{w}_{1} \geq \mathbf{v}_{1} \geq \mathbf{v}_{2} \geq \cdots \geq \mathbf{v}_{n}$. Let $\mathcal{F}_{\mathbf{v}, \mathbf{w}}$ be the variety of $n$-step partial flags, $F=\left(W \equiv F_{0} \supseteq F_{1} \supseteq \cdots \supseteq F_{n} \supseteq F_{n+1} \equiv 0\right)$, such that $\operatorname{dim} F_{i}=\mathbf{v}_{i}$ for all $1 \leq i \leq n$. The cotangent bundle $T^{*} \mathcal{F}_{\mathbf{v}, \mathbf{w}}$ of $\mathcal{F}_{\mathbf{v}, \mathbf{w}}$ can be defined as follows:

$$
T^{*} \mathcal{F}_{\mathbf{v}, \mathbf{w}}=\left\{(x, F) \in \operatorname{End}(W) \times \mathcal{F}_{\mathbf{v}, \mathbf{w}} \mid x\left(F_{i}\right) \subseteq F_{i+1} \quad \forall 0 \leq i \leq n\right\} .
$$

From Section 3.2, we assume that $W \equiv W_{1}$ is a formed space with the bilinearform $(-,-)_{W}$. Let $G(W)$ be the subgroup of $\mathrm{GL}(W)$ leaving the form invariant. In particular, if the form is a $\delta$-form, then $G(W)=\mathrm{O}_{\mathbf{w}_{1}}$ is the orthogonal group if $\delta=1$ and $G(W)=\mathrm{Sp}_{\mathbf{w}_{1}}$ is the symplectic group if $\delta=-1$. Let $\mathfrak{g}(W)$ be the Lie algebra of $G(W)$. Then we have

$$
\begin{aligned}
G(W) & =\left\{g \in \mathrm{GL}(W) \mid g g^{*}=1\right\}, \\
\mathfrak{g}(W) & =\left\{x \in \operatorname{End}(W) \mid x=-x^{*}\right\} .
\end{aligned}
$$

For each subspace $F_{i} \subseteq W$, we can define its orthogonal complement $F_{i}^{\perp}=$ $\left\{w \in W \mid\left(x, F_{i}\right)_{W}=0\right\}$. We set $F^{\perp}=\left(F_{n+1}^{\perp} \supseteq F_{n}^{\perp} \supseteq \cdots \supseteq F_{1}^{\perp} \supseteq F_{0}^{\perp}\right)$. Note that $w_{0} * \mathbf{v}=\left(\mathbf{w}_{1}-\mathbf{v}_{n}, \mathbf{w}_{1}-\mathbf{v}_{n-1}, \ldots, \mathbf{w}_{1}-\mathbf{v}_{1}\right)$. So if $F \in \mathcal{F}_{\mathbf{v}, \mathbf{w}}$, then $F^{\perp} \in \mathcal{F}_{w_{0} * \mathbf{v}, \mathbf{w}}$, thus taking $\perp$ defines an involution $\sigma_{1}: \mathcal{F}_{\mathbf{v}, \mathbf{w}} \rightarrow \mathcal{F}_{w_{0} * \mathbf{v}, \mathbf{w}}$. In the case when $w_{0} * \mathbf{v}=\mathbf{v}$, that is, $\mathbf{v}_{i}+\mathbf{v}_{n+1-i}=\mathbf{w}_{1}$ for all $1 \leq i \leq n$, the fixed point subvariety $\mathcal{F}_{\mathbf{v}, \mathbf{w}}^{\sigma_{1}}$ under $\sigma_{1}$ is a partial flag variety of the classical group $G(W)$. Its cotangent bundle is given by

$$
T^{*} \mathcal{F}_{\mathbf{v}, \mathbf{w}}^{\sigma_{1}}=\left\{(x, F) \in \mathfrak{g}(W) \times \mathcal{F}_{\mathbf{v}, \mathbf{w}}^{\sigma_{1}} \mid x\left(F_{i}\right) \subseteq F_{i+1} \quad \forall 0 \leq i \leq n\right\} .
$$

More generally, the assignment $(x, F) \mapsto\left(-x^{*}, F^{\perp}\right)$ defines an isomorphism

$$
\sigma_{1}: T^{*} \mathcal{F}_{\mathbf{v}, \mathbf{w}} \rightarrow T^{*} \mathcal{F}_{w_{0} * \mathbf{v}, \mathbf{w}} .
$$

We must prove the well-definedness of $\sigma_{1}$. We only need to show $-x^{*}\left(F_{i}^{\perp}\right) \subseteq$ $F_{i-1}^{\perp}$ for all $1 \leq i \leq n+1$. For any $u \in F_{i-1}$ and $u^{\prime} \in F_{i}^{\perp}$, one has

$$
\left(u,-x^{*}\left(u^{\prime}\right)\right)_{W}=-\left(x(u), u^{\prime}\right)_{W}=0,
$$

since $x(u) \in F_{i}$. This implies that $-x^{*}\left(u^{\prime}\right) \in F_{i-1}^{\perp}$, as required.

From the above analysis, one has

$$
T^{*} \mathcal{F}_{\mathbf{v}, \mathbf{w}}^{\sigma_{1}}=\left(T^{*} \mathcal{F}_{\mathbf{v}, \mathbf{w}}\right)^{\sigma_{1}}, \quad \text { if } w_{0} * \mathbf{v}=\mathbf{v}
$$

Note that $\mathrm{f} \mapsto \tau^{\tau} \mathrm{f}=\left(\mathrm{f}^{*}\right)^{-1}$ defines an automorphism $\tau: \mathrm{G}_{\mathbf{w}} \rightarrow \mathrm{G}_{\mathbf{w}}$. The isomorphism $\sigma_{1}$ is $\tau$-equivariant, i.e., $\sigma(\mathrm{f} .(x, F))={ }^{\tau} \mathrm{f} . \sigma_{1}(x, F)$ for all $\mathrm{f} \in \mathrm{G}_{\mathbf{w}}$ and $(x, F) \in T^{*} \mathcal{F}_{\mathbf{v}, \mathbf{w}}$. In turn, this induces a $G(W)$-action on the fixed point variety $T^{*} \mathcal{F}_{\mathbf{v}, \mathbf{w}}^{\sigma_{1}}$. 
6.2. Identification with $\sigma$-quiver varieties. In this section, we assume that $a=1$ and $\omega=w_{0}$ is the longest element in the Weyl group $\mathcal{W}$. Let $\theta: I \rightarrow I$ be the involution defined by $\theta(i)=n+1-i$ for all $1 \leq i \leq n$. Assume that the parameter $\zeta=\left(\xi, \zeta_{\mathbb{C}}\right)$ satisfies that $\zeta_{\mathbb{C}}=0, \theta(\xi)=\xi$, and $\xi_{i}>0$ for all $i \in I$. We choose the function $\varepsilon: H \rightarrow\{ \pm 1\}$ to be $\varepsilon(h)=\mathbf{o}(h)-\mathbf{i}(h)$ with the label in (78). Recall from [N94, Theorem 7.3], that is an isomorphism $\phi: \mathfrak{M}_{\zeta}(\mathbf{v}, \mathbf{w}) \rightarrow T^{*} \mathcal{F}_{\mathbf{v}, \mathbf{w}}$ of varieties given by

$$
[\mathbf{x}]=\left[x_{h}, p_{i}, q_{i}\right] \mapsto\left(q_{1} p_{1}, W_{1} \supseteq \operatorname{im} q_{1} \supseteq \operatorname{im} q_{1} y_{1} \supseteq \cdots \supseteq \operatorname{im} q_{1} y_{1} \cdots y_{n-1} \supseteq 0\right),
$$

where $y_{i}$ is the $x_{h}$ such that $\mathbf{o}(h)=i+1$ and $\mathbf{i}(h)=i$.

Theorem 6.2.1. Under Nakajima's isomorphism, the isomorphism $\sigma=\sigma_{\zeta, w_{0}}$ for $\omega=w_{0}$ (24) on quiver varieties gets identified with the isomorphism $\sigma_{1}$ (81) on the cotangent bundle of flag varieties. In particular, if $w_{0} * \mathbf{v}=\mathbf{v}$, the quiver variety $\mathfrak{S}_{\zeta}(\mathbf{v}, \mathbf{w})$ is the cotangent bundle $T^{*} \mathcal{F}_{\mathbf{v}, \mathbf{w}}^{\sigma_{1}}$.

Proof. Similar to [N94, Theorem 7.3], there is an isomorphism $\psi: \mathfrak{M}_{-\zeta}(\mathbf{v}, \mathbf{w}) \rightarrow$ $T^{*} \mathcal{F}_{w_{0} * \mathbf{v}, \mathbf{w}}$ of varieties given by

$$
[\mathbf{x}] \mapsto\left(q_{1} p_{1}, W_{1} \supseteq \operatorname{ker} x_{n-1} \cdots x_{1} p_{1} \supseteq \cdots \supseteq \operatorname{ker} x_{1} p_{1} \supseteq \operatorname{ker} p_{1} \supseteq 0\right),
$$

where $x_{i}$ stands for the $x_{h}$ with $\mathbf{o}(h)=i$ and $\mathbf{i}(h)=i+1$. The proof consists of two steps. The first step of the proof is to show that the following diagram is commutative:

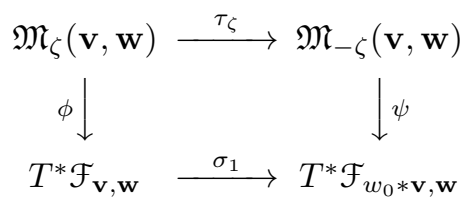

where the isomorphism $\tau_{\zeta}$ is from (17). Note that ${ }^{\tau} q_{1}{ }^{\tau} p_{1}=-\left(q_{1} p_{1}\right)^{*}$. So it suffices to show that the associated flags to $[\mathbf{x}]$ and $\left[{ }^{\tau} \mathbf{x}\right]$ can be obtained from each other via the operator $\perp$. More precisely, setting $x_{0}=y_{0}=1$, we need to show that

$$
\left(\operatorname{im} q_{1} y_{1} \cdots y_{i-1}\right)^{\perp}=\operatorname{ker}{ }^{\tau} x_{i-1} \cdots{ }^{\tau} x_{1}{ }^{\tau} p_{1}, \quad \forall 1 \leq i \leq n .
$$

Let $f_{i}=q_{1} y_{1} \cdots y_{i-1}$. Then by definition, ${ }^{\tau} x_{i-1} \cdots{ }^{\tau} x_{1}{ }^{\tau} p_{1}=(-1)^{i} f_{i}^{*}$. So for all $u \in \operatorname{im} f_{i}, u^{\prime} \in \operatorname{ker}(-1)^{i} f_{i}^{*}=\operatorname{ker} f_{i}^{*}$, there is $v_{i} \in V_{i}$ such that $f_{i}\left(v_{i}\right)=u$ and $\left(u, u^{\prime}\right)_{W_{1}}=\left(f_{i}\left(v_{i}\right), u^{\prime}\right)_{W_{1}}=\left(v_{i}, f_{i}^{*}\left(u^{\prime}\right)\right)_{V_{i}}=0$. Hence we obtain that $\operatorname{ker} f_{i}^{*} \subseteq$ $\left(\operatorname{im} f_{i}\right)^{\perp}$. Since the linear maps $q_{1}, y_{1}, \ldots, y_{i-1}$ are injective, we have that $\operatorname{dim} \operatorname{im} f_{i}+$ $\operatorname{dim} \operatorname{ker} f_{i}^{*}=\operatorname{dim} V_{i}+\operatorname{dim} W_{1}-\operatorname{dim} V_{i}=\mathbf{w}_{1}$, which implies the equality (86). This proves that the above diagram is commutative.

The second step is to show that the involution $S_{w_{0}}: \mathfrak{M}_{-\zeta}(\mathbf{v}, \mathbf{w}) \rightarrow$ $\mathfrak{M}_{\zeta}\left(w_{0} * \mathbf{v}, \mathbf{w}\right)$ commutes with the maps in (84) and (85), that is, $\psi=\phi S_{w_{0}}$. Here we use $w_{0}(-\xi)=\theta(\xi)=\xi$. Let us fix a reduced expression of the longest element $w_{0}=s_{n}\left(s_{n-1} s_{n}\right) \cdots\left(s_{1} \cdots s_{n}\right)$, so that, $S_{w_{0}}=S_{n}\left(S_{n-1} S_{n}\right) \cdots\left(S_{1} \cdots S_{n}\right)$. Observe that each time we apply $S_{i}$, the parameter on the affected quiver varieties always has a negative value at $i$. This allows us to use the definition (14). Now fix a point $[\mathbf{x}] \in \mathfrak{M}_{-\zeta}(\mathbf{v}, \mathbf{w})$, with $\mathbf{x}$ given by

$$
W_{1} \stackrel{p}{\stackrel{\leftrightarrow}{\rightleftarrows}} V_{1} \underset{y_{1}}{\stackrel{x_{1}}{\rightleftarrows}} V_{2} \underset{y_{2}}{\stackrel{x_{2}}{\rightleftarrows}} \ldots \ldots \frac{x_{n-2}}{\underset{y_{n-2}}{\rightleftarrows}} V_{n-1} \underset{y_{n-1}}{\stackrel{x_{n-1}}{\rightleftarrows}} V_{n}
$$


By applying $S_{1} \cdots S_{n}$ to $\mathbf{x}$, the point $[\mathbf{x}]$ gets sent to a point represented by

$$
\begin{aligned}
& W_{1} \stackrel{q p}{\longleftrightarrow} \operatorname{ker} x_{n-1} \cdots x_{1} p \stackrel{p}{\underset{q}{\longrightarrow}} \operatorname{ker} x_{n-1} \cdots x_{1} \stackrel{x_{1}}{\underset{y_{1}}{\longrightarrow}} \\
& \cdots \cdots \underset{y_{n-3}}{\stackrel{x_{n-3}}{\gtrless}} \operatorname{ker} x_{n-1} x_{n-2} \stackrel{x_{n-2}}{\underset{y_{n-2}}{\gtrless}} \operatorname{ker} x_{n-1}
\end{aligned}
$$

where the arrow without a name is the natural inclusion. By applying $S_{i} \cdots S_{n}$ for $i=2, \ldots, n$ consecutively, we see that the point $S_{w_{0}}([\mathbf{x}])$ is represented by

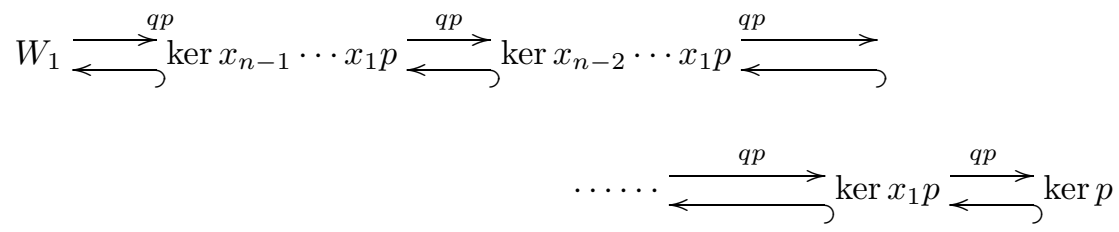

By (84) and (85), it implies immediately that $\phi S_{w_{0}}([\mathbf{x}])=\psi([\mathbf{x}])$, completing the proof.

Remark 6.2.2. The identification in Theorem 6.2.1 indicates that the geometry of general $\sigma$-quiver varieties is quite complicated as we shall see from the following remarks:

(1) In general, the quiver variety $\mathfrak{S}_{\zeta}(\mathbf{v}, \mathbf{w})$ is not connected. An example is as follows. When $\delta=1$ and $\mathbf{w}_{1}$ is even, $G(W)=\mathrm{O}_{\mathbf{w}_{1}}$ is an even orthogonal group, hence the cotangent bundle $T^{*} \mathcal{F}_{\mathbf{v}, \mathbf{w}}^{\sigma_{1}}$ has two connected/irreducible components if $n$ is odd.

(2) The morphism $\pi^{\sigma}$ is not a resolution of singularities in general. Indeed, we have a commutative diagram

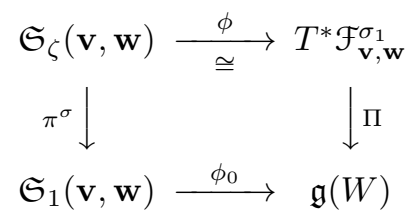

where $\Pi$ is the first projection. So we only need to know that $\Pi$ is not a resolution of singularities. But this is well known; see Fu03. For example, the morphism $\Pi: T^{*} \mathcal{F}_{\mathbf{v}, \mathbf{w}}^{\mathfrak{s p}_{6}} \rightarrow \overline{\mathcal{O}}_{\left(2^{2}, 1^{2}\right)}^{\mathfrak{s p}_{6}}$, with $\mathbf{v}=(5,1)$ and $\mathbf{w}=(6,0)$, is not a resolution of singularities since the fiber at any point of Jordan type $2^{2}, 1^{2}$ contains two points. Note that $\Pi$ is generically finite to its image.

Note that $\Pi$ factors through the affinization map of $T^{*} \mathcal{F}_{\mathbf{v}, \mathbf{w}}^{\sigma_{1}}$ which is a resolution of singularities. It is not clear if the same holds for the affinization map of $\mathfrak{S}_{\zeta}(\mathbf{v}, \mathbf{w})$.

(3) The fiber $\left(\pi^{\sigma}\right)^{-1}(0)$ is not equidimensional/lagrangian in general. Indeed, by Corollary 8.3.4 it corresponds to the fiber of a partial resolution of nilpotent Slodowy slices at $e_{0}$, which is not necessarily equidimensional/ lagrangian.

(4) In general, the variety $\mathfrak{S}_{1}(\mathbf{v}, \mathbf{w})$ is nonnormal. In $[\mathrm{KP} 82$, the orbit closure $\overline{\mathcal{O}}_{3^{2}, 1^{2}}$ in $\mathfrak{s p}_{8}$ is connected nonnormal, which is a special case of $\mathfrak{S}_{1}(\mathbf{v}, \mathbf{w})$. 


\section{NAKAJIMA-MAFFEI ISOMORPHISM AND SYMMETRY}

In this section, we assume again that the Dynkin diagram is of type $A_{n}$. We recall Nakajima-Maffei's isomorphism of the quiver varieties and partial Springer resolutions of nilpotent Slodowy slices of type A. We deduce, as preliminary results for later study, a rectangular symmetry and the column-removal and row-removal reductions in [KP81, Proposition 5.4]. (During the preparation of this paper, we noticed that these applications have appeared in [H15, Section 9].)

7.1. Nakajima-Maffei theorem. Recall from Section 6.2 that we define $\varepsilon(h)=$ $\mathbf{o}(h)-\mathbf{i}(h)$ for all arrows $h$. For any pair $(\mathbf{v}, \mathbf{w})$, we define a new pair $(\widetilde{\mathbf{v}}=$ $\left.\left(\widetilde{\mathbf{v}}_{i}\right)_{1 \leq i \leq n}, \widetilde{\mathbf{w}}=\left(\widetilde{\mathbf{w}}_{i}\right)_{1 \leq i \leq n}\right)$, where

$$
\widetilde{\mathbf{v}}_{i}=\mathbf{v}_{i}+\sum_{j \geq i+1}(j-i) \mathbf{w}_{j}, \quad \widetilde{\mathbf{w}}_{i}=\delta_{i, 1} \sum_{1 \leq j \leq n} j \mathbf{w}_{j} \quad \forall 1 \leq i \leq n .
$$

To a pair $(V, W)$ of $I$-graded vector spaces of dimension vector $(\mathbf{v}, \mathbf{w})$, we associate a new pair $(\widetilde{V}, \widetilde{W})$ of dimensional vector $(\widetilde{\mathbf{v}}, \widetilde{\mathbf{w}})$ whose $i$ th component is given by

$$
\widetilde{V}_{i}=V_{i} \oplus \bigoplus_{1 \leq h \leq j-i} W_{j}^{(h)}, \quad \widetilde{W}_{1}=\bigoplus_{1 \leq h \leq j} W_{j}^{(h)},
$$

where $W_{j}^{(h)}$ is an identical copy of $W_{j}$ for all $h$. For convenience, we set $V_{0}=0$ and $\widetilde{V}_{0}=\widetilde{W}_{1}$. With respect to the decomposition of $\widetilde{V}_{i}$, a linear map $\widetilde{x}_{i}: \widetilde{V}_{i} \rightarrow \widetilde{V}_{i+1}$ is a collection of the following four types of linear maps:

$$
X_{i}: V_{i} \rightarrow V_{i+1}, T_{i, j, h}^{V}: V_{i} \rightarrow W_{j}^{(h)}, T_{i, V}^{j^{\prime}, h^{\prime}}: W_{j^{\prime}}^{\left(h^{\prime}\right)} \rightarrow V_{i+1}, T_{i, j, h}^{j^{\prime}, h^{\prime}}: W_{j^{\prime}}^{\left(h^{\prime}\right)} \rightarrow W_{j}^{(h)},
$$

for all $j \geq i+1,1 \leq h \leq j-i$. Similarly, to give a linear map $\widetilde{y}_{i}: \widetilde{V}_{i+1} \rightarrow \widetilde{V}_{i}$ is the same as to give a collection of the following four types of linear maps for all $j \geq i+1,1 \leq h \leq j-i$ :

$$
Y_{i}: V_{i+1} \rightarrow V_{i}, S_{i, j, h}^{V}: V_{i+1} \rightarrow W_{j}^{(h)}, S_{i, V}^{j^{\prime}, h^{\prime}}: W_{j^{\prime}}^{\left(h^{\prime}\right)} \rightarrow V_{i}, S_{i, j, h}^{j^{\prime}, h^{\prime}}: W_{j^{\prime}}^{\left(h^{\prime}\right)} \rightarrow W_{j}^{(h)} .
$$

Following Maffei, we define the following numerical data:

$$
\begin{aligned}
& \operatorname{grad}\left(T_{i, j, h}^{j^{\prime}, h^{\prime}}\right)=\min \left(h-h^{\prime}+1, h-h^{\prime}+1+j^{\prime}-j\right), \\
& \operatorname{grad}\left(S_{i, j, h}^{j^{\prime}, h^{\prime}}\right)=\min \left(h-h^{\prime}, h-h^{\prime}+j^{\prime}-j\right) .
\end{aligned}
$$

Let $W_{i}^{\prime}=\bigoplus_{1 \leq h \leq j-i} W_{j}^{(h)}$ so that $\widetilde{V}_{i}=V_{i} \oplus W_{i}^{\prime}$ for all $0 \leq i \leq n$. Let $e_{i}$ : $W_{i}^{\prime} \rightarrow W_{i}^{\prime}$ be a linear map whose component $W_{j}^{(h)} \stackrel{e_{i}}{\rightarrow} W_{j}^{(h-1)}$ is equal to $\operatorname{id}_{W_{j}}$ for $2 \leq h \leq j-i$ and 0 otherwise. Let $f_{i}: W_{i}^{\prime} \rightarrow W_{i}^{\prime}$ be a linear map whose component $W_{j}^{(h)} \stackrel{f_{i}}{\rightarrow} W_{j}^{(h+1)}$ is equal to $h(j-i-h) \operatorname{id}_{W_{j}}$ for $1 \leq h \leq j-i-1$ and 0 otherwise. The triple $\left(e_{i}, f_{i},\left[e_{i}, f_{i}\right]\right)$ in $\mathfrak{s l}\left(W_{i}^{\prime}\right)$ is a Maffei $\mathfrak{s l}_{2}$-triple.

Now assume that $\zeta_{\mathbb{C}}=0$ and we write $\Lambda(V, W)$ instead of $\Lambda_{\zeta_{\mathbb{C}}}(V, W)$. We preserve the convention used in previous sections: $x_{i} / y_{i}$ stands for the map associated to the arrow $i \rightarrow i+1 / i+1 \rightarrow i$. Following Maffei, an element $\widetilde{\mathbf{x}}=\left(\widetilde{x}_{i}, \widetilde{y}_{i}, \widetilde{p}_{i}, \widetilde{q}_{i}\right) \in$ 
$\Lambda(\widetilde{V}, \widetilde{W})$, represented in the form of (89)-(90), is transversal if it satisfies the following conditions:

(r1) $\quad\left[\left.\pi_{W_{i}^{\prime}} \widetilde{y}_{i} \widetilde{x}_{i}\right|_{W_{i}^{\prime}}-e_{i}, f_{i}\right]=0$.

$$
\begin{aligned}
& T_{i, j, h}^{V}=0, \\
& T_{i, V}^{j^{\prime}, h^{\prime}}=0, \quad \text { if } h^{\prime} \neq 1,
\end{aligned}
$$$$
T_{i, j, h}^{j^{\prime}, h^{\prime}}=0, \quad \text { if } \operatorname{grad}\left(T_{i, j, h}^{j^{\prime}, h^{\prime}}\right)<0,
$$$$
T_{i, j, h}^{j^{\prime}, h^{\prime}}=0, \quad \text { if } \operatorname{grad}\left(T_{i, j, h}^{j^{\prime}, h^{\prime}}\right)=0,\left(j^{\prime}, h^{\prime}\right) \neq(j, h+1),
$$$$
T_{i, j, h}^{j^{\prime}, h^{\prime}}=\operatorname{id}_{W_{j}}, \quad \text { if } \operatorname{grad}\left(T_{i, j, h}^{j^{\prime}, h^{\prime}}\right)=0,\left(j^{\prime}, h^{\prime}\right)=(j, h+1),
$$$$
S_{i, j, h}^{j^{\prime}, h^{\prime}}=\operatorname{id}_{W_{j}}, \quad \text { if } \operatorname{grad}\left(S_{i, j, h}^{j^{\prime}, h^{\prime}}\right)=0,\left(j^{\prime}, h^{\prime}\right)=(j, h),
$$$$
S_{i, j, h}^{j^{\prime}, h^{\prime}}=0, \quad \text { if } \operatorname{grad}\left(S_{i, j, h}^{j^{\prime}, h^{\prime}}\right)=0,\left(j^{\prime}, h^{\prime}\right) \neq(j, h),
$$$$
S_{i, j, h}^{j^{\prime}, h^{\prime}}=0, \quad \text { if } \operatorname{grad}\left(S_{i, j, h}^{j^{\prime}, h^{\prime}}\right)<0,
$$$$
S_{i, j, h}^{V}=0, \quad \text { if } h \neq j-i,
$$

Proposition 7.1.1 ([M05, Lemma 19]). There is an injective morphism $\Phi: \Lambda(V, W)$ $\rightarrow \Lambda(\widetilde{V}, \widetilde{W})$ of varieties defined by the following rules. For all $\mathbf{x}=\left(x_{i}, y_{i}, p_{i}, q_{i}\right) \in$ $\Lambda(V, W)$, the element $\Phi(\mathbf{x})$ is the unique transversal element in $\Lambda(\widetilde{V}, \widetilde{W})$ that satisfies

$$
\begin{aligned}
X_{i} & =x_{i}, & Y_{i} & =y_{i}, \\
T_{i, V}^{i+1,1} & =p_{i+1}, & S_{i, i+1,1}^{V} & =q_{i+1} .
\end{aligned}
$$

Moreover, $T_{i, V}^{j, h}$ and $S_{i, j, h}^{V}$ are zero unless $j>i$, and in this case they are

$$
T_{i, V}^{j, h}=\delta_{h, 1} y_{i+1} \cdots y_{j-1} p_{j}, \quad S_{i, j, h}^{V}=\delta_{h, j-i} q_{j} x_{j-1} \cdots x_{i+1} .
$$

Assume the parameter $\xi$ satisfies that $\xi_{i}>0$ for all $i \in I$. The homomorphism $\Phi$ restricts to an injective homomorphism $\Lambda^{\xi-s s}(\mathbf{v}, \mathbf{w}) \rightarrow \Lambda^{\xi-s s}(\widetilde{\mathbf{v}}, \widetilde{\mathbf{w}})$ which is compatible with the $\mathrm{G}_{\mathbf{v}^{-}}$and $\mathrm{G}_{\mathbf{w}^{-}}$-actions on the respective varieties. Hence it induces closed immersions with $\zeta_{\mathbb{C}}=0$ :

$$
\varphi: \mathfrak{M}_{\zeta}(\mathbf{v}, \mathbf{w}) \rightarrow \mathfrak{M}_{\zeta}(\widetilde{\mathbf{v}}, \widetilde{\mathbf{w}}), \quad \varphi_{0}: \mathfrak{M}_{0}(\mathbf{v}, \mathbf{w}) \rightarrow \mathfrak{M}_{0}(\widetilde{\mathbf{v}}, \widetilde{\mathbf{w}})
$$

such that we have the following diagram:

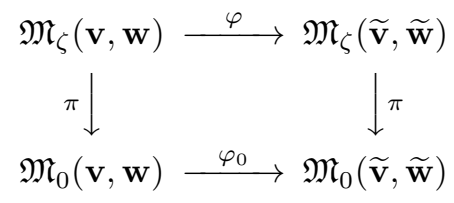

Moreover, $\varphi_{0}(0)=e_{0}$, where $e_{0}$ is in the $\mathfrak{s l}_{2}$ triple $\left(e_{0}, f_{0},\left[e_{0}, f_{0}\right]\right)$ from the paragraph below (91). Now set

$$
\mu=\left(\widetilde{\mathbf{v}}_{0}-\widetilde{\mathbf{v}}_{1}, \widetilde{\mathbf{v}}_{1}-\widetilde{\mathbf{v}}_{2}, \ldots, \widetilde{\mathbf{v}}_{n-1}-\widetilde{\mathbf{v}}_{n}, \widetilde{\mathbf{v}}_{n}\right) .
$$


We have $\mu_{i}=\mathbf{w}_{i}+\cdots+\mathbf{w}_{n}-\mathbf{v}_{i}+\mathbf{v}_{i-1}$. Reorder the entries in $\mu$ in decreasing order: $\rho_{1} \geq \rho_{2} \geq \rho_{3} \geq \cdots \geq \rho_{n+1}$ and set

$$
\mu^{\prime}=1^{\rho_{1}-\rho_{2}} 2^{\rho_{2}-\rho_{3}} \cdots n^{\rho_{n}-\rho_{n+1}}(n+1)^{\rho_{n+1}} .
$$

Let $\mathcal{O}_{\mu^{\prime}}$ be the nilpotent $\operatorname{GL}\left(\widetilde{W}_{1}\right)$-orbit in $\mathfrak{g l}\left(\widetilde{W}_{1}\right)$ whose Jordan blocks have size $\mu^{\prime}$. It is known that the closure $\overline{\mathcal{O}}_{\mu^{\prime}}$ of the orbit $\mathcal{O}_{\mu^{\prime}}$ is the image of the first projection $\Pi$ from the cotangent bundle $T^{*} \mathcal{F}_{\widetilde{\mathbf{v}}, \widetilde{\mathbf{w}}}$ to $\mathfrak{g l}\left(\widetilde{W}_{1}\right)$ so that via (84) we have a commutative diagram:

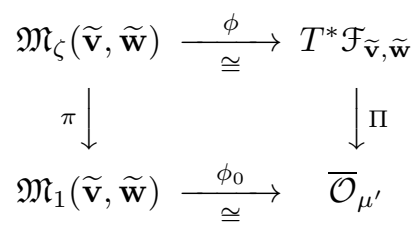

where $\mathfrak{M}_{1}(\widetilde{\mathbf{v}}, \widetilde{\mathbf{w}})$ is the image of $\pi$. Recall again Maffei's $\mathfrak{s l}_{2}$-triple $\left(e_{0}, f_{0},\left[e_{0}, f_{0}\right]\right)$ from the paragraph below (91). Following [Sl80a, Sl80b], the Slodowy transversal slice of the orbit of $e_{0}$ at $e_{0}$ is defined to be

$$
\mathcal{S}_{e_{0}}=\left\{x \in \mathfrak{g l}\left(\widetilde{W}_{1}\right) \mid x \text { is nilpotent, }\left[x-e_{0}, f_{0}\right]=0\right\} .
$$

Note that $e_{0} \in \mathcal{O}_{\lambda}$, where $\lambda=1^{\mathbf{w}_{1}} 2^{\mathbf{w}_{2}} \cdots n^{\mathbf{w}_{n}}$. For simplicity, we also say the trivial triple $(0,0,0)$ is an $\mathfrak{s l}_{2}$-triple, and in this case the Slodowy slice is the whole nilpotent cone. For convenience, we set

$$
\mathcal{S}_{\mu^{\prime}, \lambda}=\overline{\mathcal{O}}_{\mu^{\prime}} \cap \mathcal{S}_{e_{0}} \quad \text { and } \quad \widetilde{\mathcal{S}}_{\mu^{\prime}, \lambda}=\Pi^{-1}\left(\mathcal{S}_{\mu^{\prime}, \lambda}\right),
$$

where $\Pi$ is from (97). The following theorem is conjectured by Nakajima [N94, Conjecture 8.6] and proved by Maffei [M05, Theorem 8].

Theorem 7.1.2 ([M05, Theorem 8], [N94, Conjecture 8.6]). The compositions $\phi \varphi$ and $\phi_{0} \varphi_{0}$ of morphisms from (95) and (97) yield isomorphisms $\mathfrak{M}_{\zeta}(\mathbf{v}, \mathbf{w}) \simeq$ $\widetilde{\mathcal{S}}_{\mu^{\prime}, \lambda}$ and $\mathfrak{M}_{1}(\mathbf{v}, \mathbf{w}) \simeq \mathcal{S}_{\mu^{\prime}, \lambda}$, respectively. In particular, we have the following commutative diagram:

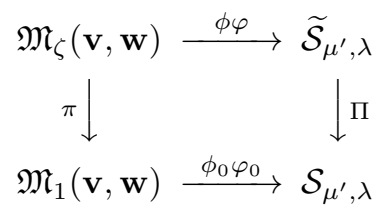

In what follows, we discuss two applications of the above remarkable theorem, which could have been stated in [M05] and has been discussed in [H15, Section 9]. We present the two applications in the following as a preparation to the analogous results in classical groups and their associated symmetric spaces.

7.2. Rectangular symmetry. In this section, we deduce a rectangular symmetry from Theorem 7.1.2. If we relabel the vertex set $I$ by $i \rightarrow \theta(i)$, where $\theta(i)=$ $n+1-i$, we can repeat the process in Section 7.1 again. In particular, we obtain an immersion

$$
\mathfrak{M}_{\zeta}(\mathbf{v}, \mathbf{w}) \rightarrow T^{*} \mathcal{F}_{\widehat{\mathbf{v}}, \widehat{\mathbf{w}}}
$$



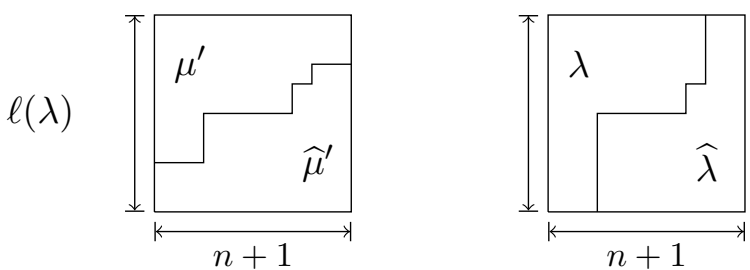

FiguRE 2. Rectangular symmetry

where the pair $(\widehat{\mathbf{v}}, \widehat{\mathbf{w}})$ is given by

(100)

$\widehat{\mathbf{w}}_{i}=\delta_{i, 1} \sum_{1 \leq j \leq n}(n+1-j) \mathbf{w}_{j}, \quad \widehat{\mathbf{v}}_{i}=\mathbf{v}_{n+1-i}+\sum_{j: j \geq i+1}(j-i) \mathbf{w}_{n+1-j} \quad \forall 1 \leq i \leq n$.

Similar to the $\mathfrak{s l}_{2}$-triple $\left(e_{0}, f_{0},\left[e_{0}, f_{0}\right]\right)$, we have an $\mathfrak{s l}_{2}$-triple $\left(\widehat{e}_{0}, \widehat{f}_{0},\left[\widehat{e}_{0}, \widehat{f}_{0}\right]\right)$ where the nilpotent element $\widehat{e}_{0}$ has the Jordan type $1^{\mathbf{w}_{\theta(1)}} 2^{\mathbf{w}_{\theta(2)}} \cdots i^{\mathbf{w}_{\theta(i)}} \cdots n^{\mathbf{w}_{\theta(n)}}$. Similar to the partition $\mu$, we also have a partition $\widehat{\mu} \equiv\left(\widehat{\mu}_{i}\right)_{1 \leq i \leq n+1}=\left(\widehat{\mathbf{v}}_{i-1}-\widehat{\mathbf{v}}_{i}\right)_{1 \leq i \leq n+1}$ determined by $\widehat{\mathbf{v}}$ and let $\widehat{\mu}^{\prime}$ be its transpose. Observe that

$$
\widehat{\mathbf{v}}_{n-i+1}-\widehat{\mathbf{v}}_{n-i+2}=\mathbf{w}_{1}+\cdots+\mathbf{w}_{i-1}+\mathbf{v}_{i}-\mathbf{v}_{i-1} .
$$

This implies that

$$
\mu_{i}+\widehat{\mu}_{n-i+2}=\mathbf{w}_{1}+\mathbf{w}_{2}+\cdots+\mathbf{w}_{n} \quad \forall 1 \leq i \leq n+1 .
$$

In particular, if the transpose $\mu^{\prime}$ is $\mu^{\prime}=1^{\mu_{1}^{\prime}} 2^{\mu_{2}^{\prime}} \cdots(n+1)^{\mu_{n+1}^{\prime}}$, then the transpose $\widehat{\mu}^{\prime}$ of $\widehat{\mu}$ is

$$
\widehat{\mu}^{\prime}=1^{\mu_{n}^{\prime}} 2^{\mu_{n-1}^{\prime}} \cdots n^{\mu_{1}^{\prime}}(n+1)^{\mu_{0}^{\prime}}=\left(i^{\mu_{\theta(i)}^{\prime}}\right)_{1 \leq i \leq n+1}, \quad \mu_{0}^{\prime}=\sum_{1 \leq i \leq n} \mathbf{w}_{i}-\sum_{1 \leq i \leq n+1} \mu_{i}^{\prime} .
$$

Hence we have a similar result as in Theorem 7.1.2 in describing the new immersion via the intersection $\mathcal{S}_{\widehat{\mu}^{\prime}, \widehat{\lambda}}=\overline{\mathcal{O}}_{\widehat{\mu}^{\prime}} \cap \mathcal{S}_{\widehat{e}_{0}}$ and its partial Springer resolution $\widetilde{\mathcal{S}}_{\widehat{\mu}^{\prime}, \widehat{\lambda}}:=$ $\Pi_{\widehat{\mathbf{v}}, \widehat{\mathbf{w}}}^{-1}\left(\mathcal{S}_{\widehat{e}_{0}}\right)$, where $\Pi_{\widehat{\mathbf{v}}, \widehat{\mathbf{w}}}$ is the natural projection similar to $\Pi$. Altogether, we have the following result.

Proposition 7.2.1. Let $(\widetilde{\mathbf{v}}, \widetilde{\mathbf{w}})$ and $(\widehat{\mathbf{v}}, \widehat{\mathbf{w}})$ be the pairs defined by (87) and (100) such that associated compositions $\mu$ and $\widehat{\mu}$ satisfy (101) (see also (102)). Then the following diagram is commutative with isomorphic horizontal maps, which sends $e_{0}$ of Jordan type $\left(i^{\mathbf{w}_{i}}\right)_{1 \leq i \leq n}$ to $\widehat{e}_{0}$ of Jordan type $\left(i^{\mathbf{w}_{\theta(i)}}\right)_{1 \leq i \leq n}$ in the base:

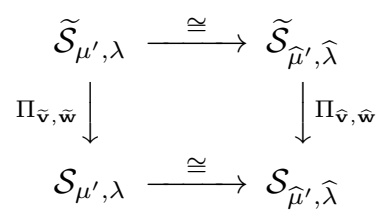

In light of (102), the partitions $\mu^{\prime}$ and $\widehat{\mu}^{\prime}$ fit into a rectangle of size $(n+1) \times$ $\sum_{1 \leq i \leq n} \mathbf{w}_{i}$. Similarly, the Jordan types $\lambda=\left(1^{\mathbf{w}_{i}}\right)_{1 \leq i \leq n}$ and $\widehat{\lambda}=\left(1^{\mathbf{w}_{\theta(i)}}\right)_{1 \leq i \leq n}$ of $e_{0}$ and $\widehat{e}_{0}$ fit into a rectangle of the same size, depicted in the following. This explains the name of the section. 

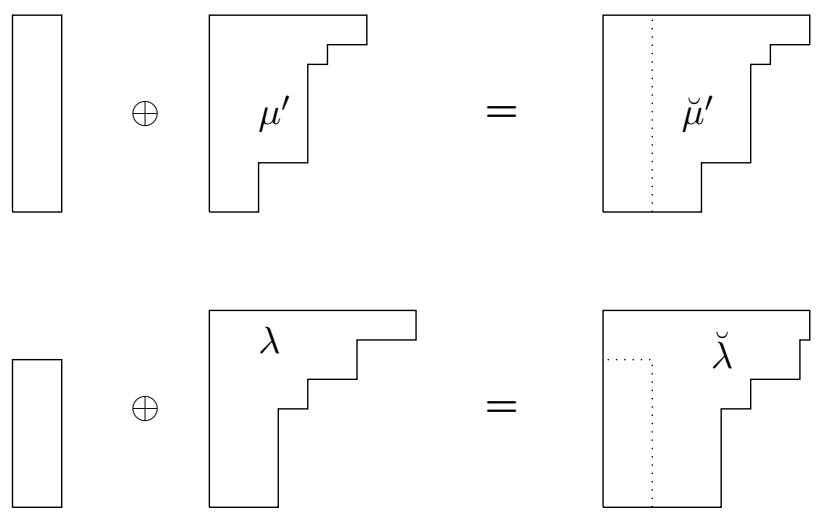

FiguRE 3. Column-removal reduction

Remark 7.2.2. Proposition 7.2.1 yields an identity on the Kostka numbers:

$$
K_{\lambda, \mu^{\prime}}=K_{\widehat{\lambda}, \widehat{\mu}^{\prime}}, \quad \text { with } \lambda=\left(i^{\mathbf{w}_{i}}\right)_{1 \leq i \leq n}, \quad \widehat{\lambda}=\left(i^{\mathbf{w}_{\theta(i)}}\right)_{1 \leq i \leq n},
$$

first proved by Briand-Orellana-Rosas in BOR15].

7.3. Column/row-removal reductions. Now we discuss the second application of Theorem 7.1.2. It is clear that the quiver varieties $\mathfrak{M}_{\zeta}(\mathbf{v}, \mathbf{w})$ are isomorphic to the quiver varieties $\mathfrak{M}_{\zeta}(\breve{\mathbf{v}}, \breve{\mathbf{w}})^{\prime}$ of Dynkin diagram $A_{n+1}$ with the dimension vectors $\breve{\mathbf{v}}, \breve{\mathbf{w}}$ given by

$$
\breve{\mathbf{v}}_{0}=0, \breve{\mathbf{v}}_{i}=\mathbf{v}_{i-1} ; \breve{\mathbf{w}}_{0}=0, \breve{\mathbf{w}}_{i}=\mathbf{w}_{i-1} \quad \forall 2 \leq i \leq n+1 .
$$

By Theorem7.1.2, we have $\mathfrak{M}_{\zeta}(\breve{\mathbf{v}}, \breve{\mathbf{w}})^{\prime} \cong \widetilde{\mathcal{S}}_{\breve{\mu}^{\prime}, \breve{\lambda}}$, where $\breve{\mu}$ has an extra entry $\sum_{1 \leq i \leq n} \mathbf{w}_{i}$ than $\mu$ and $\breve{\lambda}=\left((i+1)^{\mathbf{w}_{i}}\right)_{1 \leq i \leq n}$. It yields the following proposition.

Proposition 7.3.1. The following diagram is commutative with isomorphic horizontal maps, which sends $e_{0}$ of Jordan type $\left(i^{\mathbf{w}_{i}}\right)_{1 \leq i \leq n}$ to $\breve{e}_{0}$ of Jordan type $((i+$ $\left.1)^{\mathbf{w}_{i}}\right)_{1 \leq i \leq n}$ in the base:

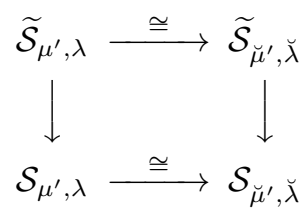

If we write the partitions involved as Young diagrams, then the partition $\lambda$ can be obtained from $\breve{\lambda}$ by removing the left-most column of $\breve{\lambda}$ via Figure 3. The partition $\mu^{\prime}$ can be obtained from $\breve{\mu}^{\prime}$ by removing part of the left-most column $\breve{\mu}^{\prime}$. Thus, Proposition 7.3.1 is a geometric version of Kraft-Procesi's column removal reduction in [KP81, Proposition 5.4].

Now we turn to provide a geometric version of Kraft-Procesi's row-removal reduction in [KP81, Proposition 4.4]. This is obtained in exactly the same spirit as that of column-removal reduction. Precisely, we can identify the variety $\mathfrak{M}_{\zeta}(\mathbf{v}, \mathbf{w})$ with another quiver variety $\mathfrak{M}_{\zeta}(\ddot{\mathbf{v}}, \ddot{\mathbf{w}})^{\prime}$ of type $A_{n+1}$, where the vectors $\ddot{\mathbf{v}}$ and $\ddot{\mathbf{w}}$ are given as follows:

$$
\ddot{\mathbf{v}}_{i}=\mathbf{v}_{i}, \ddot{\mathbf{v}}_{n+1}=0, \ddot{\mathbf{w}}_{i}=\mathbf{w}_{i}, \ddot{\mathbf{w}}_{n+1}=a \quad \forall 1 \leq i \leq n .
$$




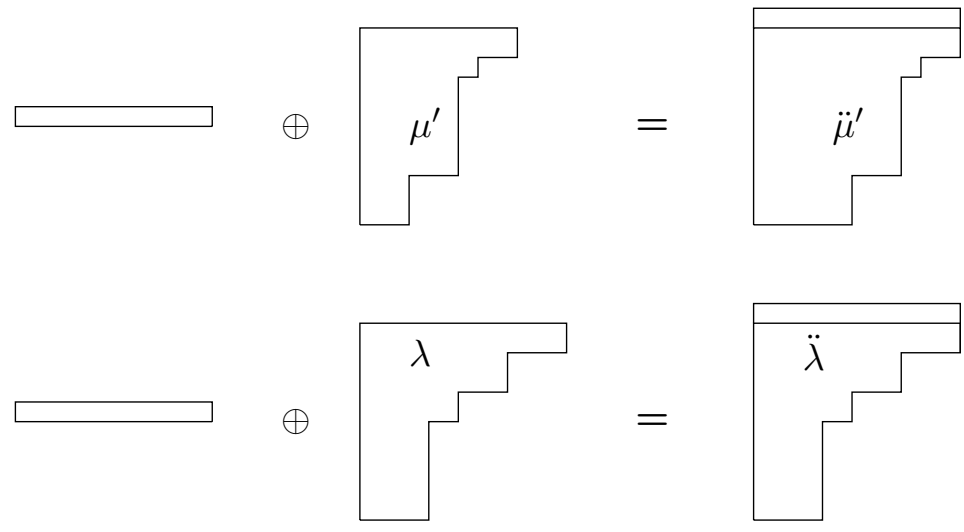

FiguRE 4. Row-removal reduction

If the associated pair of partitions to $\mathbf{w}, \mathbf{w}$ is $\left(\mu^{\prime}=\left(i^{\mu_{i}^{\prime}}\right)_{1 \leq i \leq n+1}, \lambda=\left(1^{\mathbf{w}_{i}}\right)_{1 \leq i \leq n}\right.$, then the similar one for $\ddot{\mathbf{v}}, \ddot{\mathbf{w}}$ will be given as

$$
\ddot{\mu}^{\prime}=1^{\mu_{1}^{\prime}} \cdots n^{\mu_{n}^{\prime}}(n+1)^{\mu_{n+1}^{\prime}+a}, \ddot{\lambda}=1^{\mathbf{w}_{1}} \cdots n^{\mathbf{w}_{n}}(n+1)^{a} .
$$

(Note that $\widetilde{\mathbf{v}}_{i}=\mathbf{v}_{i}+\sum_{j>i}(j-i) \mathbf{w}_{i}+(n+1-i) a$.) Therefore, by Theorem 7.1 .2 and the identification $\mathfrak{M}_{\zeta}(\mathbf{v}, \mathbf{w}) \cong \mathfrak{M}_{\zeta}(\ddot{\mathbf{v}}, \ddot{\mathbf{w}})^{\prime}$, we obtain the following proposition.

Proposition 7.3.2. The following diagram is commutative with isomorphic horizontal maps, which sends $e_{0}$ of Jordan type $\left(i^{\mathbf{w}_{i}}\right)_{1 \leq i \leq n}$ to $\ddot{e}_{0}$ of type $\left(\left(1^{\mathbf{w}_{1}} \cdots n^{\mathbf{w}_{n}}(n\right.\right.$ $+1)^{a}$ ) in the base:

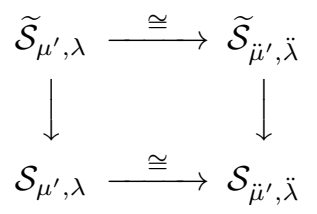

Note that the partitions $\mu^{\prime}$ and $\lambda$ can be obtained from $\ddot{\mu}^{\prime}$ and $\ddot{\lambda}$ by removing the respective first $a$ th rows in Figure 4. So Proposition 7.3.2 is a geometric version of Kraft-Procesi's row-removal reduction. Of course, by combining Propositions 7.3.1 and 7.3.2 we obtain a geometric version of the general reduction in [KP81, Proposition 3.1], which plays a critical role in the study of minimal singularities in $\mathrm{GL}_{n}$.

\section{Example II: Partial Resolutions of nilpotent Slodowy slices}

This section is devoted to the compatibility of Maffei's morphism and the isomorphism $\sigma$. From this, we see that the $\sigma$-quiver varieties encompass partial Springer resolutions of nilpotent Slodowy slices of classical groups. As a consequence, we deduce the rectangular symmetry for classical groups. The symmetry provides a natural home for the recent results of [HL14, W15] on the interactions of two-row Slodowy slices of symplectic and orthogonal groups. We also briefly discuss a geometric version of Kraft-Procesi's column-removal and row-removal reductions for classical groups in KP82, Proposition 13.5].

We again assume that $\omega=w_{0}$ and $a=1$. 
8.1. Maffei's morphism and the bilinear forms on $\widetilde{V}$ and $\widetilde{W}$. Assume that $V$ and $W$ are formed spaces with signs $\tilde{\delta}_{\mathbf{v}}$ and $\delta_{\mathbf{w}}$, respectively. Recall that we set $V_{0}=0$ and $\widetilde{V}_{0}=\widetilde{W}_{1}$. We define a nondegenerate bilinear form on $\widetilde{V}_{i}$, for all $0 \leq i \leq n$, by

$$
\begin{aligned}
\left\langle\left(v_{i}, w_{j}^{(h)}\right)_{j \geq i+h} \mid\left(v_{i}^{\prime}, u_{j}^{(h)}\right)_{j \geq i+h}\right\rangle_{\widetilde{V}_{i}}= & \left(v_{i}, v_{i}^{\prime}\right)_{V_{i}} \\
& +\sum_{j \geq i+h}(-1)^{j-i+h}\left(w_{j}^{(h)}, u_{j}^{(j-i+1-h)}\right)_{W_{j}},
\end{aligned}
$$

where $v_{i}, v_{i}^{\prime} \in V_{i}$ and $w_{j}^{(h)}, u_{j}^{(h)} \in W_{j}^{(h)}$ such that $1 \leq h \leq j-i$. The form $\langle-\mid-\rangle_{\widetilde{V}_{i}}$ on $\widetilde{V}_{i}$ may not be a $\delta$-form. However, if the signs $\tilde{\delta}_{\mathbf{v}}$ and $\delta_{\mathbf{w}}$ alternate, it turns out to be the case.

Lemma 8.1.1. If the sign $\delta_{\mathbf{w}}$ is $\Gamma$-alternating as in Proposition 3.2 .2 , then $\widetilde{W}_{1}$ is $a(-1)^{i+1} \delta_{\mathbf{w}, i}$-form (for each $i$ ). If further $\tilde{\delta}_{\mathbf{v}}$ is $\Gamma$-alternating and $\tilde{\delta}_{\mathbf{v}, i} \delta_{\mathbf{w}, i}=-1$ for all $i$, then the form on $\widetilde{V}_{i}$ is a $\tilde{\delta}_{\mathbf{v}, i}$-form for all $1 \leq i \leq n$.

Proof. We only need to observe that for a fixed $j_{0}$ such that $j_{0}-i$ is even (resp., odd), then the restriction of $\langle-\mid-\rangle_{\widetilde{V}_{i}}$ to $\bigoplus_{1 \leq h \leq j_{0}-i} W_{j_{0}}^{(h)}$ is a $-\delta_{\mathbf{w}, j_{0}}$-form (resp., $\delta_{\mathbf{w}, j_{0}}$-form). Specifically, we have

$$
\begin{aligned}
& \left\langle\left(u_{j_{0}}^{(h)}\right)_{1 \leq h \leq j_{0}-i} \mid\left(w_{j_{0}}^{(h)}\right)_{1 \leq h \leq j_{0}-i}\right\rangle_{\widetilde{V}_{i}} \\
& \quad=(-1)^{j_{0}-i+1} \delta_{\mathbf{w}, j_{0}}\left\langle\left(w_{j_{0}}^{(h)}\right)_{1 \leq h \leq j_{0}-i} \mid\left(u_{j_{0}}^{(h)}\right)_{1 \leq h \leq j_{0}-i}\right\rangle_{\widetilde{V}_{i}},
\end{aligned}
$$

for all elements $\left(w_{j_{0}}^{(h)}\right)_{1 \leq h \leq j_{0}-i},\left(u_{j_{0}}^{(h)}\right)_{1 \leq h \leq j_{0}-i}$ in $\bigoplus_{1 \leq h \leq j_{0}-i} W_{j_{0}}^{(h)}$.

Let $T^{\natural}: \widetilde{V}_{j} \rightarrow \widetilde{V}_{i}$ denote the right adjoint of the linear map $T: \widetilde{V}_{i} \rightarrow \widetilde{V}_{j}$, with respect to the forms on $\widetilde{V}_{i}$ and $\widetilde{V}_{j}$. In particular, the right adjoint of a point in $\Lambda(\widetilde{V}, \widetilde{W})$ represented as the collection of linear maps in (89) and (90) satisfies the following:

$$
X_{i}^{\natural}=X_{i}^{*}, \quad Y_{i}^{\natural}=Y_{i}^{*},
$$

$$
\begin{aligned}
\left(T_{i, V}^{i+1,1}\right)^{\natural} & =\left(T_{i, V}^{i+1,1}\right)^{*}, & \left(S_{i, i+1,1}^{V}\right)^{\natural} & =\left(S_{i, i+1,1}^{V}\right)^{*}, \\
\left(T_{i, j, h}^{j^{\prime}, h^{\prime}}\right)^{\natural} & =(-1)^{j-j^{\prime}+h-h^{\prime}-1}\left(T_{i, j, h}^{j^{\prime}, h^{\prime}}\right)^{*}, & \left(S_{i, j, h}^{j^{\prime}, h^{\prime}}\right)^{\natural} & =(-1)^{j-j^{\prime}+h-h^{\prime}+1}\left(S_{i, j, h}^{j^{\prime}, h^{\prime}}\right)^{*} .
\end{aligned}
$$

Indeed, the identities in the first two rows are due to the fact that the signs of the forms on $V_{i}$ and $W_{i+1}^{(1)}$ at vertex $i$ remain unchanged. For simplicity, we write $t=T_{i, j, h}^{j^{\prime}, h^{\prime}}$ and then

$$
(-1)^{j-(i+1)+h}(t(u), v)_{W_{j}}=\langle t(u) \mid v\rangle_{\widetilde{V}_{i+1}}=\left\langle u \mid t^{\natural}(v)\right\rangle_{\widetilde{V}_{i}}=(-1)^{j^{\prime}-i+h^{\prime}}\left(u, t^{\natural}(v)\right)_{W_{j^{\prime}}},
$$

for all $u \in W_{j^{\prime}}^{\left(h^{\prime}\right)}$ and $v \in W_{j}^{(h)}$. So we have $t^{\natural}=(-1)^{j-j^{\prime}+h-h^{\prime}-1} t^{*}$, which is the first identity in the last row of (108). Identities in the second column of (108) are proved in a similar way.

By the definition (15), we have an automorphism $\widetilde{\tau}: \Lambda(\widetilde{V}, \widetilde{W}) \rightarrow \Lambda(\widetilde{V}, \widetilde{W})$ with respect to the forms on $\widetilde{V}$ and $\widetilde{W}$. We have the following compatibility result of Maffei's morphism and the automorphisms $\tau$ in (15) and its analog $\widetilde{\tau}$ on $\Lambda(\widetilde{V}, \widetilde{W})$. 
Proposition 8.1.2. The following diagram commutes:

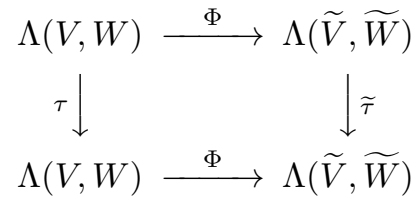

Proof. Fix a point $\mathbf{x}=\left(x_{i}, y_{i}, p_{i}, q_{i}\right) \in \Lambda(V, W)$. By definition, the point $\Phi \tau(\mathbf{x})$ is a point in $\Lambda(\widetilde{V}, \tilde{W})$ determined by the transversal conditions (s1)-(s5), (t) - (t5), (r1) and the following:

$$
\begin{aligned}
X_{i} & =-y_{i}^{*}, & Y_{i} & =x_{i}^{*}, \\
T_{i, V}^{i+1,1} & =-q_{i+1}^{*}, & S_{i, i+1,1}^{V} & =p_{i+1}^{*} .
\end{aligned}
$$

So it suffices to show that the point $\widetilde{\tau} \Phi(\mathbf{x})$ satisfies the transversal conditions and the above.

We put a superscript $\widetilde{\tau}$ on the upper left for the decomposition (89)-(90) of $\widetilde{\tau} \Phi(\mathbf{x})$ with respect to the fixed decompositions of $\widetilde{V}$ and $\widetilde{W}$. By (108), the point $\widetilde{\tau} \Phi(\mathbf{x})$ satisfies

$$
\begin{aligned}
\widetilde{\tau} X_{i} & =-Y_{i}^{\natural}=-y_{i}^{*}, & \widetilde{\tau} Y_{i} & =X_{i}^{\natural}=x_{i}^{*}, \\
{ }^{\tau} T_{i, V}^{i+1,1} & =-\left(S_{i, i+1,1}^{V}\right)^{\natural}=-q_{i+1}^{*}, & \widetilde{\tau} S_{i, i+1,1}^{V} & =\left(T_{i, V}^{i+1,1}\right)^{\natural}=p_{i+1}^{*} .
\end{aligned}
$$

Hence it remains to show that the point $\widetilde{\tau} \Phi(\mathbf{x})$ satisfies the transversal conditions. The conditions (ti) and (si) for $1 \leq i \leq 5$ for $\widetilde{\tau} \Phi(\mathbf{x})$ follow from the conditions (si) and (ti) for $\Phi(\mathbf{x})$, respectively. More precisely, for (t1), we have

$$
\widetilde{\tau} T_{i, j, h}^{V}=-\left(S_{i, V}^{j, j-i+1-h}\right)^{\natural}=0 .
$$

For (t2), we notice that $h^{\prime} \neq 1$ if and only if $\left(j^{\prime}-i+1-h^{\prime}\right) \neq j^{\prime}-i$. So by the (s2) of $\Phi(\mathbf{x})$,

$$
\widetilde{\tau} T_{i, V}^{j^{\prime}, h^{\prime}}=-\left(S_{i, j^{\prime}, j^{\prime}-i+1-h^{\prime}}^{V}\right)^{\natural}=0 .
$$

This shows that $\widetilde{\tau} \Phi(\mathbf{x})$ satisfies the condition (t2). For (t3), we observe that

$$
\operatorname{grad}\left(T_{i, j, h}^{j^{\prime}, h^{\prime}}\right)=\operatorname{grad}\left(S_{i, j^{\prime}, j^{\prime}-i+1-h^{\prime}}^{j, j-(i+1)+1-h}\right),
$$

and $\left(j^{\prime}, h^{\prime}\right) \neq(j, h+1)$ if and only if $(j, j-i-h) \neq\left(j^{\prime}, j^{\prime}-i+1-h^{\prime}\right)$. Thanks to these observations and the (S3)-(S4) of $\Phi(\mathbf{x})$, it leads to

$$
\widetilde{\tau}_{i, j, h}^{j^{\prime}, h^{\prime}}=-\left(S_{i, j^{\prime}, j^{\prime}-i+1-h^{\prime}}^{j, j-(i+1)+1-h}\right)^{\natural}=0,
$$

if either $\operatorname{grad}\left(\widetilde{\tau} T_{i, j, h}^{j^{\prime}, h^{\prime}}\right)<0$ or $\operatorname{grad}\left(\widetilde{{ }^{\tau}} T_{i, j, h}^{j^{\prime}, h^{\prime}}\right)=0$ and $\left(j^{\prime}, h^{\prime}\right) \neq(j, h+1)$. If $\operatorname{grad}\left({ }^{\tau} T_{i, j, h}^{j^{\prime}, h^{\prime}}\right)=0$ and $(j, j-i-h)=\left(j^{\prime}, j^{\prime}-i+1-h^{\prime}\right)$, then

$$
\left(S_{i, j^{\prime}, j^{\prime}-i+1-h^{\prime}}^{j, j-(i+1)+1-h}\right)^{\natural}=-\left(S_{i, j^{\prime}, j^{\prime}-i+1-h^{\prime}}^{j, j-(i+1)+1-h}\right)^{*}=-\left(\operatorname{id}_{W_{j}}\right)^{*}=-\mathrm{id}_{W_{j}},
$$

where the second equality is from the (s5) of $\Phi(\mathbf{x})$. So we have

$$
{ }^{\widetilde{\tau}} T_{i, j, h}^{j^{\prime}, h^{\prime}}=\operatorname{id}_{W_{j}}, \quad \text { if } \operatorname{grad}\left({ }^{\widetilde{\tau}} T_{i, j, h}^{j^{\prime}, h^{\prime}}\right)=0,\left(j^{\prime}, h^{\prime}\right)=(j, h+1) .
$$

By now, it has been shown that the point $\widetilde{\tau} \Phi(\mathbf{x})$ satisfies the conditions (t1)- (t5). By an entirely similar argument, we can check that the point $\widetilde{\tau} \Phi(\mathbf{x})$ satisfies the

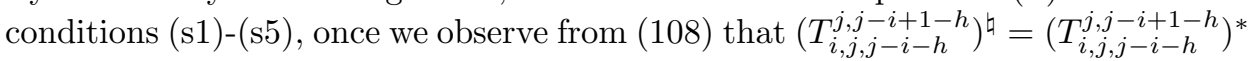
for (55). 
Recall that the automorphism $\widetilde{\tau}$ on $\mathfrak{s l}\left(W_{i}^{\prime}\right)$, compatible with $\widetilde{\tau}$ on $\Lambda(\widetilde{\mathbf{v}}, \widetilde{\mathbf{w}})$, is given by $x \mapsto-x^{\natural}$. By a similar analysis as above, one can check that ${ }^{\widetilde{\tau}} e_{i}=e_{i}$ and ${ }^{\widetilde{\tau}} f_{i}=f_{i}$. This implies that the point $\widetilde{\tau} \Phi(\mathbf{x})$ satisfies the last condition (r1), completing the proof.

By Proposition 8.1.2, (18) and (95), we have the following.

Proposition 8.1.3. Assume that $\zeta=(\xi, 0)$ with $\xi$ satisfying that $\xi_{i}>0$ for all $i \in I$. Then the automorphism $\tau_{\zeta}$ in (17) and the embeddings $\varphi$ and $\varphi_{0}$ in (95) are compatible. Specifically, we have the following commutative diagram.

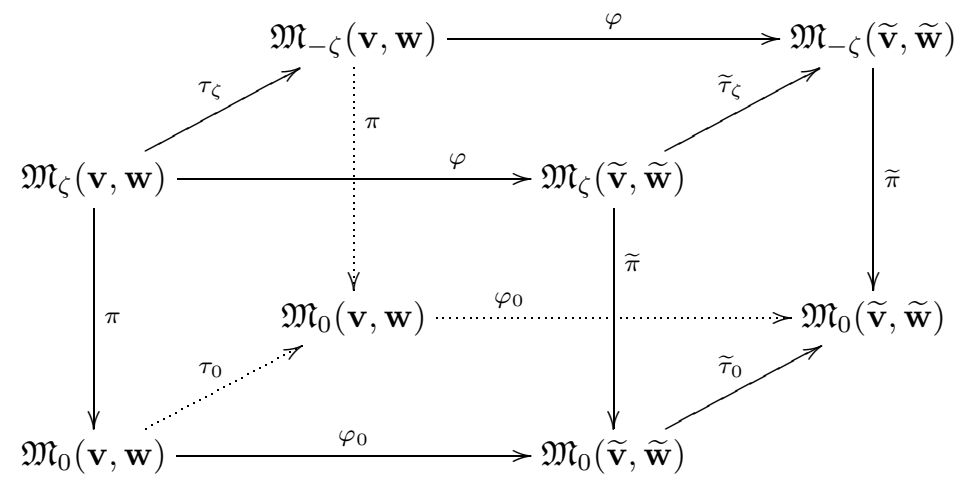

8.2. Maffei's morphism and reflection functors. In this section, we show the compatibility of Maffei's morphism and the reflection functors on quiver varieties in Section 3.1 .

Recall that to a pair $(\mathbf{v}, \mathbf{w})$ and a fixed vertex $i \in I$, we associate a new pair $\left(\mathbf{v}^{\prime}, \mathbf{w}\right)=\left(s_{i} *_{\mathbf{w}} \mathbf{v}, \mathbf{w}\right)$ in Section 3.1 To the same pair, we attach a third pair $(\widetilde{\mathbf{v}}, \widetilde{\mathbf{w}})$ in Section 7.1. Now apply the operation in (3.1) to $(\widetilde{\mathbf{v}}, \widetilde{\mathbf{w}})$; we have $\left((\widetilde{\mathbf{v}})^{\prime}, \widetilde{\mathbf{w}}\right)=$ $\left(s_{i} *_{\widetilde{\mathbf{w}}} \widetilde{\mathbf{v}}, \widetilde{\mathbf{w}}\right)$, while applying the procedure in Section 7.1 yields $\left(\widetilde{\mathbf{v}}^{\prime}, \widetilde{\mathbf{w}}\right)$. We now compare $(\widetilde{\mathbf{v}})^{\prime}$ and $\widetilde{\mathbf{v}}^{\prime}$. If $j \neq i$, then $(\widetilde{\mathbf{v}})_{j}^{\prime}=(\widetilde{\mathbf{v}})_{j}=\left(\widetilde{\mathbf{v}}^{\prime}\right)_{j}$. With the convention $\widetilde{\mathbf{v}}_{0}=\widetilde{\mathbf{w}}_{1}$, the two vectors $(\widetilde{\mathbf{v}})^{\prime}$ and $\widetilde{\mathbf{v}}^{\prime}$ coincide at $j=i$ by the following computation:

$$
(\widetilde{\mathbf{v}})_{i}^{\prime}=\widetilde{\mathbf{v}}_{i-1}+\widetilde{\mathbf{v}}_{i+1}-\widetilde{\mathbf{v}}_{i}=\mathbf{v}_{i}^{\prime}+\sum_{j \geq i+1}(j-i) \mathbf{w}_{j}=\widetilde{\mathbf{v}}_{i}^{\prime}
$$

As a result, we have $(\widetilde{\mathbf{v}})^{\prime}=\widetilde{\mathbf{v}}^{\prime}$.

Similar to Section 3.1. we fix a triple $\left(V, V^{\prime}, W\right)$ of dimension vector $\left(\mathbf{v}, \mathbf{v}^{\prime}, \mathbf{w}\right)$ and $V_{j}=V_{j}^{\prime}$ for all $j \neq i$. We define $\widetilde{V}, \widetilde{W}$, and $\widetilde{V}^{\prime}$ as in Section 7.1. In particular, $\widetilde{V}_{j}^{\prime}=\widetilde{V}_{j}=V_{j} \oplus \bigoplus_{k \geq j+1,1 \leq h \leq k-j} W_{k}^{(h)}$ if $j \neq i$ and $\widetilde{V}_{i}^{\prime}=V_{i}^{\prime} \oplus \bigoplus_{k \geq i+1,1 \leq k \leq k-i} W_{k}^{(h)}$.

Recall diagram (12) from Section 3.1. We write $\Lambda^{\xi-s s}(V, W)$ for $\Lambda_{\zeta_{\mathbb{C}}}^{\xi-s s}(V, W)$ when $\zeta_{\mathbb{C}}=0$. With the above preparation, we are ready to state the compatibility of Maffei's morphism $\Phi$ in Proposition 7.1.1 with the reflection functor diagram (12).

Proposition 8.2.1. Fix $i \in I$. Assume that $\zeta=(\xi, 0)$ and there is $w \in W$ such that $w(\xi)_{j}>0$ for all $j \in I$ (equivalently, here is $w \in W$ such that $w(\xi)_{j}<0$ 
$\forall j \in I)$. The following diagram commutes:

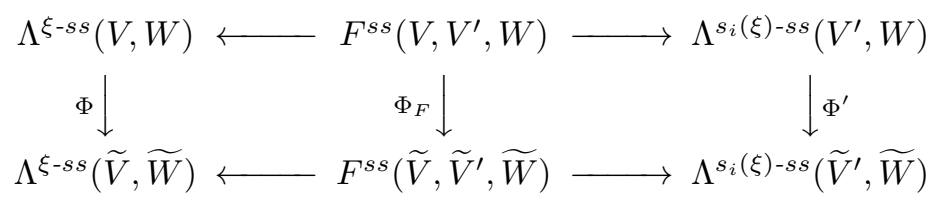

where the rows are (12) attached to the triples $\left(V, V^{\prime}, W\right)$ and $(\widetilde{V}, \widetilde{V}, \widetilde{W})$, the vertical morphisms $\Phi$ and $\Phi^{\prime}$ on the left and right are the restriction of the morphism from Proposition [7.1.1, and the middle one is defined to be $\Phi_{F}\left(\mathbf{x}, \mathbf{x}^{\prime}\right)=$ $\left(\Phi(\mathbf{x}), \Phi^{\prime}\left(\mathbf{x}^{\prime}\right)\right)$.

Proof. We first assume that $\xi_{j}<0$ for all $j<0$. Then the morphism $\Phi$ is welldefined by [M05, Lemma 19]. In this case, it suffices to show that the pair $\left(\widetilde{\mathbf{x}}, \widetilde{\mathbf{x}}^{\prime}\right) \equiv$ $\left(\Phi(\mathbf{x}), \Phi^{\prime}\left(\mathbf{x}^{\prime}\right)\right)$ satisfies the conditions (R1)-( $(\mathbb{R} 4)$. In light of [M02, Lemmas 28, 30], this shows that the maps $\Phi^{\prime}$ and $\Phi_{F}$ are well-defined, which is not obvious since the $\widetilde{\mathbf{x}}$ and $\widetilde{\mathbf{x}}^{\prime}$ are defined inductively.

We define a new element $\widetilde{\mathbf{y}} \in \mathbf{M}\left(\widetilde{V}^{\prime}, \widetilde{W}\right)$, as a package of linear maps with respect to the decompositions of $\widetilde{V}, \widetilde{V}^{\prime}$, and $\widetilde{W}$, such that a linear map in $\widetilde{\mathbf{y}}$ is defined to be its counterpart in $\widetilde{\mathbf{x}}^{\prime}$ if it involves $V_{i}^{\prime}$, or its counterpart in $\widetilde{\mathbf{x}}$ otherwise.

If we can show that the pair $(\widetilde{\mathbf{x}}, \widetilde{\mathbf{y}})$ satisfies the conditions (R1)-(R4), then we have $\widetilde{\mathbf{y}} \in \Lambda^{s_{i}(\xi)-s s}\left(\widetilde{V}^{\prime}, \widetilde{W}\right)$ by $[$ 02, Lemma 28, Lemma 30]. By the definition of $\widetilde{\mathbf{y}}$ and [M05, Lemma 18], we immediately see that $\widetilde{\mathbf{y}}=\widetilde{\mathbf{x}}^{\prime}$, which implies the well-definedness of $\Phi_{F}$.

It remains to show that the pair $(\widetilde{\mathbf{x}}, \widetilde{\mathbf{y}})$ satisfies (R1) $(\mathrm{R} 4)$. Clearly from the definitions, the pair satisfies $(\overline{\mathrm{R} 3})$ and $(\overline{\mathrm{R} 4})$. We now prove that the sequence in (R1) for the pair $(\widetilde{\mathbf{x}}, \widetilde{\mathbf{y}})$ is a complex, i.e., $b_{i}(\widetilde{\mathbf{x}}) a_{i}(\widetilde{\mathbf{y}})=0$. We consider the restriction to the subspace $W_{i}^{\prime}=\bigoplus_{j \geq i+1,1 \leq h \leq j-i} W_{j}^{(h)}$ of $\widetilde{V}^{\prime}=V_{i}^{\prime} \oplus W_{i}^{\prime}$, and we get

$$
\left.b_{i}(\widetilde{\mathbf{x}}) a_{i}(\widetilde{\mathbf{y}})\right|_{W_{i}^{\prime}}=\left.b_{i}(\widetilde{\mathbf{x}}) a_{i}(\widetilde{\mathbf{x}})\right|_{W_{i}^{\prime}}=-\left.\mu_{i}(\widetilde{\mathbf{x}})\right|_{W_{i}^{\prime}}=0 .
$$

We then consider the restriction of $b_{i}(\widetilde{\mathbf{x}}) a_{i}(\widetilde{\mathbf{y}})$ to $V_{i}^{\prime}$ in two cases. The first one is to consider $\left.\pi_{V_{i}} b_{i}(\widetilde{\mathbf{x}}) a_{i}(\widetilde{\mathbf{y}})\right|_{V_{i}^{\prime}}$, where $\pi_{V_{i}}$ is the project to the component $V_{i}$. Any component in $\left.\pi_{V_{i}} b_{i}(\widetilde{\mathbf{x}}) a_{i}(\widetilde{\mathbf{y}})\right|_{V_{i}^{\prime}}$ passing through $W_{i+1}^{\prime}$ is zero. The possible nonzero component of the linear map $\left.\pi_{V_{i}} b_{i}(\widetilde{\mathbf{x}}) a_{i}(\tilde{\mathbf{y}})\right|_{V_{i}^{\prime}}$ passing through $W_{i-1}^{\prime}$ is when it factors through its subspace $W_{i}^{(1)}$, which is $p_{i} q_{i}^{\prime}$ if we use the notation $\mathbf{x}=\left(x_{i}, y_{i}, p_{i}, q_{i}\right)$ and $\mathbf{x}^{\prime}=\left(x_{i}^{\prime}, y_{i}^{\prime}, p_{i}^{\prime}, q_{i}^{\prime}\right)$. Hence we have

$$
\left.\pi_{V_{i}} b_{i}(\widetilde{\mathbf{x}}) a_{i}(\widetilde{\mathbf{y}})\right|_{V_{i}^{\prime}}=b_{i}(\mathbf{x}) a_{i}\left(\mathbf{x}^{\prime}\right)=0 .
$$

The second case is to consider the component $\left.\pi_{W_{i}^{\prime}} b_{i}(\widetilde{\mathbf{x}}) a_{i}(\widetilde{\mathbf{y}})\right|_{V_{i}^{\prime}}$. In this case, the possible nonzero component factored through the map $\left.\pi_{W_{i}^{\prime}} b_{i}(\widetilde{\mathbf{x}})\right|_{W_{i-1}^{\prime}}$ is when it factors through the linear map $T_{i-1, j, j-i}^{j, j-i+1}: W_{j}^{(j-i+1)} \rightarrow W_{j}^{(j-i)}$, and it equals $S_{i-1, j, j-i+1}^{V^{\prime}}\left(\widetilde{\mathbf{x}}^{\prime}\right)$ of $\widetilde{\mathbf{x}}^{\prime}$. On the other hand, the possible nonzero component factored through the map $\left.\pi_{W_{i}^{\prime}} b_{i}(\widetilde{\mathbf{x}})\right|_{W_{i+1}^{\prime}}$ is when it factors through the linear map $-S_{i, j, j-i}^{V}(\widetilde{\mathbf{x}})$ of $\widetilde{\mathbf{x}}$ and it equals $-S_{i, j, j-i}^{V}(\widetilde{\mathbf{x}}) x_{i}^{\prime}=-S_{i-1, j, j-i+1}^{V^{\prime}}\left(\widetilde{\mathbf{x}}^{\prime}\right)$. The two sums to zero, and therefore we obtain

$$
\left.\pi_{W_{i}^{\prime}} b_{i}(\widetilde{\mathbf{x}}) a_{i}(\widetilde{\mathbf{y}})\right|_{V_{i}^{\prime}}=0 .
$$

Altogether, it confirms that the sequence in (R1) for $(\widetilde{\mathbf{x}}, \widetilde{\mathbf{y}})$ is a complex. 
It remains to show that the map $a_{i}(\widetilde{\mathbf{y}})$ is injective for the pair $(\widetilde{\mathbf{x}}, \widetilde{\mathbf{y}})$ to satisfy (1). Clearly $\left.a_{i}(\widetilde{\mathbf{y}})\right|_{V_{i}^{\prime}}=\left.a_{i}\left(\widetilde{\mathbf{x}}^{\prime}\right)\right|_{V_{i}^{\prime}}$ is injective. The fact that $S_{i-1, j, h}^{j, h}=\operatorname{id}_{W_{j}}$ implies that $\left.a_{i}(\widetilde{\mathbf{y}})\right|_{W_{i}^{\prime}}$ is injective. So $a_{i}(\widetilde{\mathbf{y}})$ is injective, and thus $(\mathbb{R} 1)$ holds for $(\widetilde{\mathbf{x}}, \widetilde{\mathbf{y}})$.

Now we show that the pair $(\widetilde{\mathbf{x}}, \widetilde{\mathbf{y}})$ satisfies the last condition $(\overline{\mathrm{R} 2})$. We observe from the definition of $\widetilde{\mathbf{y}}$ that $\left.a_{i}(\widetilde{\mathbf{x}})\right|_{W_{i}^{\prime}} \pi_{W_{i}^{\prime}} b_{i}(\widetilde{\mathbf{x}})-\left.a_{i}(\widetilde{\mathbf{y}})\right|_{W_{i}^{\prime}} \pi_{W_{i}^{\prime}} b_{i}(\widetilde{\mathbf{y}})=0$. So it is reduced to show that $\left.a_{i}(\widetilde{\mathbf{x}})\right|_{V_{i}} \pi_{V_{i}} b_{i}(\widetilde{\mathbf{x}})-\left.a_{i}(\widetilde{\mathbf{y}})\right|_{V_{i}^{\prime}} \pi_{V_{i}^{\prime}} b_{i}(\widetilde{\mathbf{y}})=0$. By using the (t1) and (s1) of $\widetilde{\mathbf{x}}$ and $\widetilde{\mathbf{y}}$, we have

$$
\begin{aligned}
{\left.\left[\left.a_{i}(\widetilde{\mathbf{x}})\right|_{V_{i}} \pi_{V_{i}} b_{i}(\widetilde{\mathbf{x}})-\left.a_{i}(\widetilde{\mathbf{y}})\right|_{V_{i}^{\prime}} \pi_{V_{i}^{\prime}} b_{i}(\widetilde{\mathbf{y}})\right]\right|_{W_{i+1}^{\prime}}=0, } \\
\pi_{W_{i+1}^{\prime}}\left[\left.a_{i}(\widetilde{\mathbf{x}})\right|_{V_{i}} \pi_{V_{i}} b_{i}(\widetilde{\mathbf{x}})-\left.a_{i}(\widetilde{\mathbf{y}})\right|_{V_{i}^{\prime}} \pi_{V_{i}^{\prime}} b_{i}(\widetilde{\mathbf{y}})\right]=0 .
\end{aligned}
$$

Moreover, in light of (t2) and (s2), it yields

$$
\begin{aligned}
& \left.\pi_{W_{i-1}^{\prime}}\left[\left.a_{i}(\widetilde{\mathbf{x}})\right|_{V_{i}} \pi_{V_{i}} b_{i}(\widetilde{\mathbf{x}})-\left.a_{i}(\widetilde{\mathbf{y}})\right|_{V_{i}^{\prime}} \pi_{V_{i}^{\prime}} b_{i}(\widetilde{\mathbf{y}})\right]\right|_{W_{i-1}^{\prime}} \\
& =\left.\bigoplus_{j>i} S_{i, j, j-i}^{V} \pi_{V_{i+1}}\left[a_{i}(\mathbf{x}) b_{i}(\mathbf{x})-a_{i}\left(\mathbf{x}^{\prime}\right) b_{i}\left(\mathbf{x}^{\prime}\right)\right]\right|_{V_{i+1}} \bigoplus_{j^{\prime}>i} T_{i, V}^{j^{\prime}, 1}=0 .
\end{aligned}
$$

The following vanishing results can be obtained in a similar manner:

$$
\begin{gathered}
\left.\pi_{U_{i}}\left[\left.a_{i}(\widetilde{\mathbf{x}})\right|_{V_{i}} \pi_{V_{i}} b_{i}(\widetilde{\mathbf{x}})-\left.a_{i}(\widetilde{\mathbf{y}})\right|_{V_{i}^{\prime}} \pi_{V_{i}^{\prime}} b_{i}(\widetilde{\mathbf{y}})\right]\right|_{U_{i}}=a_{i}(\mathbf{x}) b_{i}(\mathbf{x})-a_{i}\left(\mathbf{x}^{\prime}\right) b_{i}\left(\mathbf{x}^{\prime}\right)=0, \\
\left.\pi_{U_{i}}\left[\left.a_{i}(\widetilde{\mathbf{x}})\right|_{V_{i}} \pi_{V_{i}} b_{i}(\widetilde{\mathbf{x}})-\left.a_{i}(\widetilde{\mathbf{y}})\right|_{V_{i}^{\prime}} \pi_{V_{i}^{\prime}} b_{i}(\widetilde{\mathbf{y}})\right]\right|_{W_{i-1}^{\prime}} \\
=\left.\left[a_{i}(\mathbf{x}) b_{i}(\mathbf{x})-a_{i}\left(\mathbf{x}^{\prime}\right) b_{i}\left(\mathbf{x}^{\prime}\right)\right]\right|_{V_{i+1}} \underset{j^{\prime}>i}{\bigoplus} T_{i, V}^{j^{\prime}, 1}=0, \\
\left.\pi_{W_{i-1}^{\prime}}\left[\left.a_{i}(\widetilde{\mathbf{x}})\right|_{V_{i}} \pi_{V_{i}} b_{i}(\widetilde{\mathbf{x}})-\left.a_{i}(\widetilde{\mathbf{y}})\right|_{V_{i}^{\prime}} \pi_{V_{i}^{\prime}} b_{i}(\widetilde{\mathbf{y}})\right]\right|_{U_{i}} \\
=\bigoplus_{j>i} S_{i, j, j-i}^{V} \pi_{V_{i+1}}\left[a_{i}(\mathbf{x}) b_{i}(\mathbf{x})-a_{i}\left(\mathbf{x}^{\prime}\right) b_{i}\left(\mathbf{x}^{\prime}\right)\right]=0 .
\end{gathered}
$$

These analyses imply that the pair $(\widetilde{\mathbf{x}}, \widetilde{\mathbf{y}})$ satisfies $(\underline{\mathrm{R} 2})$. This finishes the proof under the assumption that $\xi_{j}<0$ for all $j \in I$.

As a result, we see that the map $\Phi$ is well-defined if $\xi$ is chosen so that the entries in $s_{i}(\xi)$ are positive. Now the general case is obtained by an induction on the length of $w$. The proposition is thus proved.

Let $\widetilde{S}_{i}: \mathfrak{M}_{\zeta}(\widetilde{\mathbf{v}}, \widetilde{\mathbf{w}}) \rightarrow \mathfrak{M}_{s_{i}(\zeta)}\left(s_{i} * \widetilde{\mathbf{v}}, \widetilde{\mathbf{w}}\right)$ be the reflection functor in (14) defined for the pair $(\widetilde{\mathbf{v}}, \widetilde{\mathbf{w}})$. As a consequence of Proposition 8.2.1, we have the compatibility of the reflection functor $S_{i}$ (14) and Maffei's morphism $\varphi$ (95).

Proposition 8.2.2. Fix $i \in I$. Assume that $\zeta=(\xi, 0)$ and there is an element $w \in W$ such that $w(\xi)_{j}>0$ for all $j \in I$, or $w(\xi)_{j}<0$ for all $j \in I$. Then the reflection functor $S_{i}$ in (14) and the imbedding $\varphi$ in (95) are compatible with each other. More precisely, we have the following commutative diagram:

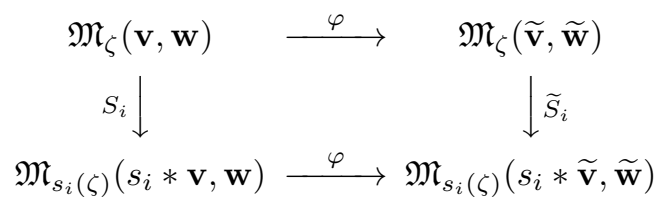

8.3. $\sigma$-quiver varieties and partial resolutions of nilpotent Slodowy slices. For the pair $(\widetilde{\mathbf{v}}, \widetilde{\mathbf{w}})$, let $\widetilde{\sigma}: \mathfrak{M}_{\zeta}(\widetilde{\mathbf{v}}, \widetilde{\mathbf{w}}) \rightarrow \mathfrak{M}_{-w_{0}(\zeta)}\left(w_{0} * \widetilde{\mathbf{v}}, \widetilde{\mathbf{w}}\right)$ denote the isomorphism defined by (24). By combining Propositions 8.1.3 and 8.2.2, we obtain the compatibility of the isomorphism $\sigma$ (24) and the immersion $\varphi$ (95). 
Proposition 8.3.1. Assume that the parameter $\zeta=0$ or $\zeta=(\xi, 0)$ satisfies that $\xi_{i}>0$ for all $i \in I$. Then the isomorphism $\sigma=\sigma_{\zeta, w_{0}}$ for $\omega=w_{0}$ in (24) and the imbedding $\varphi$ in (95) are compatible, so that we have the following commutative diagram:

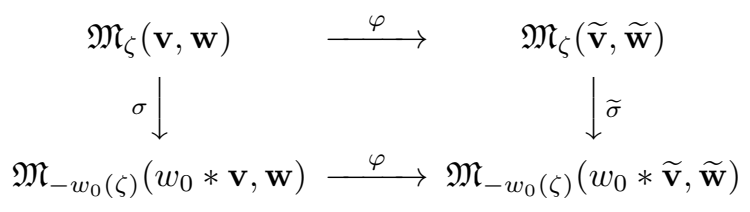

By an abuse of notation, let $\widetilde{\sigma}$ be the isomorphism on $T^{*} \mathcal{F}_{\widetilde{\mathbf{v}}}, \widetilde{\mathbf{w}}$ defined by (81) with respect to the form on $\widetilde{W}$ in (107), which is compatible with the isomorphism on $\mathfrak{M}_{\xi}(\widetilde{\mathbf{v}}, \widetilde{\mathbf{w}})$ under the same notation by Theorem 6.2.1. So we have the following corollary.

Corollary 8.3.2. Under the setting of Proposition 8.3.1, the isomorphism $\sigma$ is compatible with the isomorphism $\widetilde{\sigma}$ on $T^{*} \mathcal{F}_{\widetilde{\mathbf{v}}, \widetilde{\mathbf{w}}}$ via $\phi \varphi$.

Recall the varieties $\widetilde{\mathcal{S}}_{\mu^{\prime}, \lambda}$ and $\mathcal{S}_{\mu^{\prime}, \lambda}$ from (97). We have the following analogue for classical groups of the Nakajima-Maffei theorem.

Theorem 8.3.3. Assume that $\zeta=(\xi, 0)$ with $\xi_{i}>0$ for all $i \in I$ and $\theta(\xi)=\xi$. Assume further that $w_{0} * \mathbf{v}=\mathbf{v}$. The compositions $\phi \varphi$ and $\phi_{0} \varphi_{0}$ of morphisms from (95) and (97) yield isomorphisms $\left(\sigma=\sigma_{\zeta, w_{0}}\right.$ for $\left.\omega=w_{0}\right)$

$$
\mathfrak{S}_{\zeta}(\mathbf{v}, \mathbf{w}) \cong \widetilde{\mathcal{S}}_{\mu^{\prime}, \lambda}^{\widetilde{\sigma}} \quad \text { and } \quad \mathfrak{S}_{1}(\mathbf{v}, \mathbf{w}) \cong \mathcal{S}_{\mu^{\prime}, \lambda}^{\widetilde{\sigma}}
$$

In particular, we have the following commutative diagram:

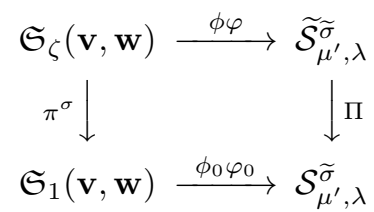

Proof. The isomorphisms are consequences of Corollary 8.3 .2 and Theorem 7.1.2,

We now derive some specific results from Theorem 8.3.3 assuming that $W$ is a formed space with alternating sign $\delta_{\mathbf{w}}$. Recall from Lemma 8.1.1 that if $\delta_{\mathbf{w}}$ alternates, then $\widetilde{W}$ is a $\delta$-form with $\delta=\delta_{\mathbf{w}, 1}$. Precisely, we write $\widetilde{\mathcal{S}}_{\mu^{\prime}, \lambda}^{\widetilde{w}_{1}}$ for $\widetilde{\mathcal{S}}_{\mu^{\prime}, \lambda}^{\widetilde{\sigma}}$ if the form associated to $\widetilde{\sigma}$ is a symmetric form. Similarly there are notation $\mathcal{S}_{\mu^{\prime}, \lambda}^{\mathfrak{0}}$, $\widetilde{\mathcal{S}}_{\mu^{\prime}, \lambda}^{\mathfrak{s p}}$, and $\mathcal{S}_{\mu^{\prime}, \lambda}^{\mathfrak{s p}}$.

Corollary 8.3.4. If $\delta_{\mathbf{w}}$ alternates, i.e., $\delta_{\mathbf{w}, i} \delta_{\mathbf{w}, i+1}=-1$ for all $1 \leq i \leq n-1$, then the isomorphisms in (116) read as follows:

$$
\begin{aligned}
\mathfrak{S}_{\zeta}(\mathbf{v}, \mathbf{w}) \cong \widetilde{\mathcal{S}}_{\mu^{\prime}, \lambda}^{\mathfrak{o}_{\tilde{w}}}, \mathfrak{S}_{1}(\mathbf{v}, \mathbf{w}) \cong \mathcal{S}_{\mu^{\prime}, \lambda}^{\mathfrak{o}_{\tilde{\mathrm{w}}},}, & \text { if } \delta_{\mathbf{w}, i}=(-1)^{i+1} . \\
\mathfrak{S}_{\zeta}(\mathbf{v}, \mathbf{w}) \cong \widetilde{\mathcal{S}}_{\mu^{\prime}, \lambda}^{\mathfrak{s p},}, \mathfrak{S}_{1}(\mathbf{v}, \mathbf{w}) \cong \mathcal{S}_{\mu^{\prime}, \lambda}^{\mathfrak{s p},}, & \text { if } \delta_{\mathbf{w}, i}=(-1)^{i} .
\end{aligned}
$$


8.4. Rectangular symmetry for classical groups. By combining the rectangular symmetry for general linear groups in Section 7.2 and Theorem 8.3 .3 , we can obtain a rectangular symmetry for classical groups. We repeat the process in Section 7.2 We have an immersion

$$
\mathfrak{S}_{\zeta}(\mathbf{v}, \mathbf{w}) \rightarrow T^{*} \mathcal{F}_{\widehat{\mathbf{v}}, \widehat{\mathbf{w}}}^{\widehat{\sigma}},
$$

where the pair $(\widehat{\mathbf{v}}, \widehat{\mathbf{w}})$ is in (100) and $\widehat{\sigma}$ is the automorphism induced from a form of the vector space of dimension $\widehat{\mathbf{w}}_{1}$ defined similar to (107). There is a similar result as Theorem 8.3.3 in describing the new immersion via the intersection $\mathcal{S}_{\widehat{\mu}^{\prime}, \widehat{\lambda}}^{\widehat{\sigma}}=\overline{\mathcal{O}}_{\widehat{\mu}^{\prime}}^{\widehat{\sigma}} \cap \mathcal{S}_{\widehat{e}_{0}}^{\widehat{\sigma}}$ and its partial Springer resolution $\widetilde{\mathcal{S}}_{\widehat{\mu}^{\prime}, \widehat{\lambda}}^{\widehat{\sigma}}=\Pi_{\widehat{\mathbf{v}}, \widehat{\mathbf{w}}}^{-1}\left(\mathcal{S}_{\widehat{e}_{0}}\right)^{\widehat{\sigma}}$. Thus, we have the following counterpart of Proposition 7.2.1.

Theorem 8.4.1. Let $(\widetilde{\mathbf{v}}, \widetilde{\mathbf{w}})$ and $(\widehat{\mathbf{v}}, \widehat{\mathbf{w}})$ be the pairs defined by (87) and (100) such that associated compositions $\mu$ and $\widehat{\mu}$ satisfy (101) (see also (102)). Then the following diagram is commutative with isomorphic horizontal maps, which sends $e_{0}$ of Jordan type $\left(i^{\mathbf{w}_{i}}\right)_{1 \leq i \leq n}$ to $\widehat{e}_{0}$ of Jordan type $\left(i^{\mathbf{w}_{\theta(i)}}\right)_{1 \leq i \leq n}$ in the base:

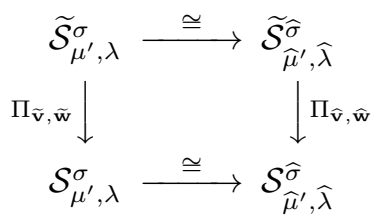

Remark 8.4.2. Perhaps the most important case of Theorem 8.4.1 and Corollary 8.3.4 is the rectangular symmetry between geometries of $\mathrm{Sp}_{2 w}$ and $\mathrm{O}_{2 w^{\prime}}$ for various $w$ and $w^{\prime}$ and respective Lie algebras $\mathfrak{s p}_{2 w}$ and $\mathfrak{o}_{2 w^{\prime}}$. Specifically, assume that $n$ is even and $\delta_{\mathbf{w}}$ alternates with $\delta_{\mathbf{w}, i}=(-1)^{i}$. Then Theorem 8.4.1 (120) yields the following commutative diagram:

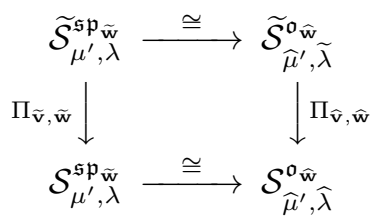

Further, the associated Springer fibers $\mathcal{F}_{\widetilde{\mathbf{v}}, \tilde{\widetilde{w}} ; e_{0}}^{\mathfrak{s}}$ and $\mathcal{F}_{\widehat{\mathbf{v}}, \widehat{\mathbf{w}} ; \widehat{e}_{0}}^{\mathfrak{o}}$ of $\Pi_{\widetilde{\mathbf{v}}, \widetilde{\mathbf{w}}}$ and $\Pi_{\widehat{\mathbf{v}}, \widehat{\mathbf{w}}}$ are isomorphic:

$$
\mathcal{F}_{\widetilde{\mathbf{v}}, \widehat{\mathbf{w}} ; e_{0}}^{\mathfrak{s} \mathfrak{p}_{\tilde{\mathbf{w}}}} \cong \mathcal{F}_{\widehat{\mathbf{v}}, \widehat{\mathfrak{w}} ; \widehat{e}_{0}}^{\mathfrak{O}}
$$

In the case when $n$ is even and $\delta_{\mathbf{w}}$ alternates with $\delta_{\mathbf{w}, i}=(-1)^{i+1}$, one has a similar diagram with the pair $\left(\mathfrak{s p}_{\widetilde{\mathbf{w}}}, \mathfrak{o}_{\widehat{\mathbf{w}}}\right)$ replaced by $\left(\mathfrak{o}_{\widetilde{\mathbf{w}}}, \mathfrak{s p}_{\widehat{\mathbf{w}}}\right)$. In a similar manner for $n$ being odd, one gets similar diagrams with the pair $\left(\mathfrak{s p}_{\widetilde{\mathbf{w}}}, \mathfrak{o}_{\widehat{\mathbf{w}}}\right)$ replaced by either $\left(\mathfrak{s p}_{\widetilde{\mathbf{w}}}, \mathfrak{s p}_{\widehat{\mathbf{w}}}\right)$ or $\left(\mathfrak{o}_{\widetilde{\mathbf{w}}}, \mathfrak{o}_{\widehat{\mathbf{w}}}\right)$.

Now we single out a special pair of $(\mathbf{v}, \mathbf{w})$ for (121) and (122) in the following example and relate it to the works of Henderson-Licata HL14 and Wilbert W15] (see also [ES12]).

Example 8.4.3. Fix an integer $k$ such that $0<k \leq n-k$. Define $\mathbf{w}^{\dagger}$ by $\mathbf{w}_{i}^{\dagger}=$ $\delta_{i, k}+\delta_{i, n-k}$. Recall that $n$ is even and set $r=n / 2$ for convenience. Let $\mathbf{v}^{\dagger}$ be a vector defined as follows:

\begin{tabular}{|c|c|c|c|c|c|c|c|c|c|c|c|c|c|c|}
\hline$i$ & 1 & 2 & $\cdots$ & $k-1$ & $k$ & $\cdots$ & $r$ & $r+1$ & $\cdots$ & $n-k$ & $n-k+1$ & $\cdots$ & $n-1$ & $n$ \\
\hline $\mathbf{v}_{i}^{\dagger}$ & 1 & 2 & $\cdots$ & $k-1$ & $k$ & $\cdots$ & $k$ & $k+1$ & $\cdots$ & $k+1$ & $k$ & $\cdots$ & 2 & 1 \\
\hline
\end{tabular}


With respect to the pair $\left(\mathbf{v}^{\dagger}, \mathbf{w}^{\dagger}\right)$, the data in (121) read

$$
\begin{aligned}
\widetilde{\mathbf{v}}^{\dagger}=(n-1, \ldots, r+1, r, r, r-1, \ldots, 1), & \widetilde{\mathbf{w}}_{i}^{\dagger}=\delta_{i, 1} n, \\
\widehat{\mathbf{v}}^{\dagger}=(n+1, \ldots, r+3, r+2, r, r-1, \ldots, 1), & \widehat{\mathbf{w}}_{i}^{\dagger}=\delta_{i, 1}(n+2) .
\end{aligned}
$$

Note that $\widetilde{\mathbf{v}}^{\dagger}$ has an extra $r$, while $r+1$ is missing from $\widehat{\mathbf{v}}^{\dagger}$. In particular, we have

$$
\begin{array}{ll}
\mu^{\prime}=n^{1}, & e_{0} \in \mathcal{O}_{k^{1}(n-k)^{1}} \cap \mathfrak{s p}_{n}, \\
\widehat{\mu}^{\prime}=1^{1}(n+1)^{1}, & \widehat{e}_{0} \in \mathcal{O}_{(k+1)^{1}(n+1-k)^{1}} \cap \mathfrak{o}_{n+2} .
\end{array}
$$

The bottom row of (121) reads as the following, which is Corollary 5.2 in [HL14]:

$$
\mathcal{S}_{n^{1}, k^{1}(n-k)^{1}}^{\mathfrak{s p}_{n}} \cong \mathcal{S}_{1^{1}(n+1)^{1},(k+1)^{1}(n+1-k)^{1}}^{\mathfrak{o}_{n+2}} .
$$

Note that both sides in (123) are empty unless $k$ is even or $k=n-k$.

Observe the $\mathcal{F}_{\widetilde{\mathbf{v}}^{\dagger}, \widetilde{\mathbf{w}}^{\dagger}}^{\mathfrak{w}_{n}}$ is the complete flag variety of $\mathrm{Sp}_{n}$. Hence the left-hand side of (122) is the Springer fiber, say $\mathcal{B}_{e_{0}}^{\mathfrak{s p}_{n}}$, of $e_{0}$. In light of the fact that the complete flag variety of $\mathrm{O}_{2}$ consists of two points, the $\mathcal{F}_{\widehat{\mathbf{v}}^{\dagger}, \widehat{\mathbf{w}}^{\dagger}}$ is isomorphic to a connected component, say $\mathcal{B}^{\mathfrak{s o}_{n+2}}$, of the complete flag variety of $\mathrm{O}_{n+2}$. So we get $T^{*} \mathcal{B}^{\mathfrak{s o}_{n+2}} \cong T^{*} \mathcal{F}_{\widehat{\mathbf{v}}^{\dagger}, \widehat{\mathbf{w}}^{\dagger}}^{\mathfrak{o}_{n+2}}$. So the right-hand side of the (122) for $\left(\mathbf{v}^{\dagger}, \mathbf{w}^{\dagger}\right)$ is exactly the Springer fiber $\mathcal{B}_{\widehat{e}_{0}}^{\mathfrak{s o}_{n+2}}$ of $\widehat{e}_{0}$. Thus, the equality (122) is transformed into the following isomorphism, which is Theorem B in [W15]:

$$
\mathcal{B}_{e_{0}}^{\mathfrak{s p}_{n}} \cong \mathcal{B}_{\widehat{e}_{0}}^{\mathfrak{s o}{ }_{n+2}} \text {. }
$$

Finally, the top row of (121) implies the following isomorphism of the Springer resolutions of the nilpotent Slodowy slices in (123), which proves a conjecture in [HL14, 1.3]:

$$
\widetilde{\mathcal{S}}_{n^{1}, k^{1}(n-k)^{1}}^{\mathfrak{s p}_{n}} \cong \widetilde{\mathcal{S}}_{1^{1}(n+1)^{1},(k+1)^{1}(n+1-k)^{1^{\prime}}} .
$$

The isomorphism (125) together with [HL14, Theorem 1.2] implies a conjecture by McGerty and Lusztig on the relationship between type D Nakajima varieties and Slodowy varieties in [HL14, 1.3].

8.5. Column/row removal reductions for classical groups. Now we investigate the classical counterpart of the geometric column/row removal reductions in Section 7.3. Recall from Section 7.3 that we have the isomorphism $\mathfrak{M}_{\zeta}(\mathbf{v}, \mathbf{w}) \cong$ $\mathfrak{M}_{\zeta}(\breve{\mathbf{v}}, \breve{\mathbf{w}})^{\prime}$. If $V$ and $W$ are formed spaces with signs $\tilde{\delta}_{\mathbf{v}}$ and $\left.\delta_{\mathbf{w}}\right)$, respectively, then the associated vector space $\breve{V}$ of dimension $\breve{\mathbf{v}}$ (resp., $\breve{W}$ ) naturally inherit one from $\tilde{\delta}_{\mathbf{v}}\left(\right.$ resp. $\left.\delta_{\mathbf{w}}\right)$. So we have an automorphism $\sigma^{\prime}$ on $\mathfrak{M}_{\zeta}(\breve{\mathbf{v}}, \breve{\mathbf{w}})^{\prime}$. In particular, we have the following geometric incarnation of [KP82, Proposition 13.5].

Proposition 8.5.1. There is $\mathcal{S}_{\mu^{\prime}, \lambda}^{\widetilde{\sigma}} \cong \mathcal{S}_{\breve{\mu}^{\prime}, \breve{\lambda}}^{\widetilde{\sigma}^{\prime}}$, where $\widetilde{\sigma}$ is from (8.3) and $\widetilde{\sigma}^{\prime}$ is defined similarly.

Since the definition of $\sigma^{\prime}$ involves the longest Weyl group element of the Dynkin diagram of type $A_{n+1}$, it is not immediately clear how to compare $\widetilde{\mathcal{S}}_{\mu^{\prime}, \lambda}^{\widetilde{\sigma}}$ and $\widetilde{\mathcal{S}}_{\widetilde{\mu}^{\prime}, \breve{\lambda}}^{\widetilde{\sigma}^{\prime}}$. In general, the two varieties are not isomorphic. However, in the case when $\left(\mu^{\prime}, \lambda\right)$ and $\left(\breve{\mu}^{\prime}, \breve{\lambda}\right)$ satisfy the conditions in Theorem 8.4.1, they are isomorphic. Example 8.4.3 is such a case. Similarly, we have the following counterpart of Proposition 7.3.2.

Proposition 8.5.2. Suppose that the pair $\left(\ddot{\mu}^{\prime}, \ddot{\lambda}\right)$ is defined by (105). There is an isomorphism $\mathcal{S}_{\mu^{\prime}, \lambda}^{\widetilde{\sigma}} \cong \mathcal{S}_{\ddot{\mu}^{\prime}, \ddot{\lambda}}^{\ddot{\lambda}}$, where $\widetilde{\sigma}$ is from (8.3) and $\ddot{\sigma}$ is defined similarly. 
By Propositions 8.5.1 and 8.5.2, one has a geometric version of Theorem 12.3 in KP82].

\section{Fixed-Points and CATEgorical Quotients}

In this section, we consider quiver varieties $\mathfrak{M}_{0}\left(\mathbf{v}^{0}, \mathbf{w}^{0}\right)^{\tau}$ of a general Dynkin graph for those pairs of formed spaces $\left(V^{0}, W^{0}\right)$ of dimension vectors $\left(\mathbf{v}^{0}, \mathbf{w}^{0}\right)$, and signs $\tilde{\delta}_{\mathbf{v}^{0}}$ and $\delta_{\mathbf{w}^{0}}$ are chosen to be alternating, i.e., $\tilde{\delta}_{\mathbf{v}^{0}, i} \delta_{\mathbf{w}^{0}, i}=-1$ for all $i \in I$ and $\tilde{\delta}_{\mathbf{v}^{0}, \mathbf{i}(h)} \tilde{\delta}_{\mathbf{v}^{0}, \mathbf{o}(h)}=-1$ for all $h \in H$. In Remark 9.2.4, we will consider $\mathfrak{M}_{0}\left(\mathbf{v}^{0}, \mathbf{w}^{0}\right)^{a \tau}$. We show that there is a closed immersion from Kraft-ProcesiNakajima's construction KP82, N94 via categorical quotients to $\sigma$-quiver varietes $\mathfrak{M}_{0}(\mathbf{v}, \mathbf{w})^{a \tau}$.

9.1. Polynomial invariants on $\mathbf{M}\left(\mathbf{v}^{0}, \mathbf{w}^{0}\right)^{\tau}$. Recall the automorphism $\tau$ on $\mathbf{M}\left(\mathbf{v}^{0}, \mathbf{w}^{0}\right)$ from (15) and $\mathrm{G}_{\mathbf{v}^{0}}$ from (44). Let $\mathrm{G}_{\mathbf{v}^{0}}^{\tau}=\left\{g \in \mathrm{G}_{\mathbf{v}^{0}} \mid g_{i} g_{i}^{*}=1\right\}$, and let $\mathbf{M}^{\tau}=\mathbf{M}\left(\mathbf{v}^{0}, \mathbf{w}^{0}\right)^{\tau}$ be the variety of $\tau$-fixed points in $\mathbf{M}\left(\mathbf{v}^{0}, \mathbf{w}^{0}\right)$. We are interested in finding a set of generators for the algebra $R^{\mathrm{G}_{\mathbf{v}^{0}}^{\tau}}$ of $\mathrm{G}_{\mathbf{v}^{0}}^{\tau}$-invariant regular functions on $\mathbf{M}^{\tau}$. Following Lusztig, we consider the following elements in $R^{\mathrm{G}_{\mathbf{v}^{0}}^{\tau}}$. A sequence $h_{1}, \ldots, h_{s}$ of arrows in $H$ is called a path if $\mathbf{i}\left(h_{i}\right)=\mathbf{o}\left(h_{i+1}\right)$ for all $1 \leq i \leq s-1$. It is called a cycle if it further satisfies $\mathbf{i}\left(h_{s}\right)=\mathbf{o}\left(h_{1}\right)$. For a cycle $h_{1}, \ldots, h_{s}$ in $H$, we define a $\mathrm{G}_{\mathbf{v}^{0}}^{\tau}$-invariant function $\operatorname{tr}_{h_{1}, \ldots, h_{s}}$ on $\mathbf{M}^{\tau}$ by

$$
\operatorname{tr}_{h_{1}, \cdots, h_{s}}(\mathbf{x})=\operatorname{trace}\left(x_{h_{s}} x_{h_{s-1}} \cdots x_{h_{1}}\right) \quad \forall \mathbf{x} \in \mathbf{M}^{\tau} .
$$

For any path $h_{1}, \ldots, h_{s} \in H$ and a linear form $\chi$ on $\operatorname{Hom}\left(W_{\mathbf{o}\left(h_{1}\right)}^{0}, W_{\mathbf{i}\left(h_{s}\right)}^{0}\right)$, we define a $\mathrm{G}_{\mathbf{v}}^{\tau}$-invariant function $\chi_{h_{1}, \ldots, h_{s}}$ on $\mathbf{M}^{\tau}$ by

$$
\chi_{h_{1}, \ldots, h_{s}}(\mathbf{x})=\chi\left(q_{\mathbf{i}\left(h_{s}\right)} x_{h_{s}} x_{h_{s-1}} \cdots x_{h_{1}} p_{\mathbf{o}\left(h_{1}\right)}\right) \quad \forall \mathbf{x} \in \mathbf{M}^{\tau} .
$$

The following theorem is an analogue for classical groups of [L98, Theorem 1.3].

Theorem 9.1.1. Assume that the signs $\tilde{\delta}_{\mathbf{v}^{0}}$ and $\delta_{\mathbf{w}^{0}}$ alternate. The algebra $R_{\mathbf{v}^{0}}^{\mathrm{G}^{0}}$ is generated by the functions of the forms (126) and (127). In particular, the algebra of $\mathrm{G}_{\mathbf{v}^{0}}^{\tau}$-invariant regular functions on $\Lambda\left(\mathbf{v}^{0}, \mathbf{w}^{0}\right)^{\tau}$ in (16) is generated by the restriction of the functions (126) and (127) to $\Lambda\left(\mathbf{v}^{0}, \mathbf{w}^{0}\right)^{\tau}$.

The remaining part of this section is devoted to the proof of Theorem 9.1.1. The proof is modeled on that of [L98, Theorem 1.3] with slight modifications. Instead of the results on tensor invariants for general linear groups, we need a similar result on tensor invariants for classical groups as follows. Let $E$ be a $\delta$-formed space with the form $(-,-)_{E}$, and let $G(E)$ be the group of isometries with respect to the form $(-,-)_{E}$. If $n$ is even and $x=\left\{\left(i_{1}, j_{1}\right), \ldots,\left(i_{n / 2}, j_{n / 2}\right)\right\}$ is a set of ordered pairs such that $\left\{i_{1}, j_{1}, \ldots, i_{n / 2}, j_{n / 2}\right\}=\{1, \ldots, n\}$, we define the following $G(E)$ invariant linear forms on $T=E^{\otimes n}$ by

$$
f_{x}\left(e_{1} \otimes \cdots \otimes e_{n}\right)=\prod_{k=1}^{n / 2}\left(e_{i_{k}}, e_{j_{k}}\right) \quad \forall e_{1} \otimes \cdots \otimes e_{n} \in E^{\otimes n} .
$$

Proposition 9.1.2 ([W39]). The space of $G(E)$-invariant linear forms on the tensor space $T$ is zero when $n$ is odd, and it is spanned by the forms $f_{x}$ (128) for various $x$ when $n$ is even. 
Now we begin to prove Theorem 9.1.1. For simplicity we write $V$ and $W$ for $V^{0}$ and $W^{0}$, respectively, in the proof. Recall the function $\varepsilon: H \rightarrow\{ \pm 1\}$ from Section 2.2. Let $\Omega=\varepsilon^{-1}(1)$ and we set

$$
\mathbf{M}_{\Omega}=\mathbf{M}_{\Omega}\left(\mathbf{v}^{0}, \mathbf{w}^{0}\right)=\bigoplus_{h \in \Omega} \operatorname{Hom}\left(V_{\mathbf{o}(h)}, V_{\mathbf{i}(h)}\right) \oplus \bigoplus_{i \in I} \operatorname{Hom}\left(V_{i}, W_{i}\right) .
$$

Since the parameters $\tilde{\delta}_{\mathbf{v}^{0}}$ and $\delta_{\mathbf{w}^{0}}$ alternate, there is an isomorphism

$$
\mathbf{M}^{\tau} \cong \mathbf{M}_{\Omega}
$$

given by projection. After fixing a basis $\mathcal{B}_{i}$ for $W_{i}$ and identifying $V_{i}$ and $V_{i}^{*}$ via the forms, we have

$$
\mathbf{M}_{\Omega} \cong \bigotimes_{h \in \Omega} V_{\mathbf{o}(h)} \otimes V_{\mathbf{i}(h)} \oplus \bigoplus_{i \in I, b \in \mathcal{B}_{i}} V_{i, b}
$$

where $V_{i, b}$ is a copy of $V_{i}$ indexed by $b$.

Following Lustig, it is enough to show that the space of $\mathrm{G}_{\mathbf{v}^{0}}^{\tau}$-invariant regular functions on $\mathbf{M}^{\tau}$ of homogenous degree $n$ is spanned by various products of functions of the form (126) and (127). Thanks to [L98, Lemma 1.4], it is reduced to show that this is also the case for the space of $G_{\mathbf{v}^{0}}^{\tau}$-invariant linear forms on $\left(\mathbf{M}^{\tau}\right)^{\otimes n}$. To this end, it is further reduced to study the $\mathrm{G}_{\mathbf{v}^{0}}^{\tau}$-invariant linear forms on the tensor space $T=E_{1} \otimes \cdots \otimes E_{n}$, where $E_{i}$ is either $V_{\mathbf{o}(h)} \otimes V_{\mathbf{i}(h)}$ or $V_{i, b}$. Write $T=\bigotimes_{i \in I} E^{i}$, where $E^{i}$ is the tensor product of all $V_{i}$ in $T$. In light of [L98, Lemma 1.5], the $\mathrm{G}_{\mathbf{v}^{0}}^{\tau}$-invariant linear forms on $T$ are the tensor products of $G\left(V_{i}\right)$-invariant linear forms on $E^{i}$. If $T$ can be decomposed as the tensor product of components of the following forms,

$$
\begin{aligned}
V_{\mathbf{o}\left(h_{1}\right)} \otimes V_{\mathbf{i}\left(h_{1}\right)}^{\otimes 2} \otimes V_{\mathbf{i}\left(h_{2}\right)}^{\otimes 2} \otimes & \cdots \otimes V_{\mathbf{i}\left(h_{s-1}\right)}^{\otimes 2} \otimes V_{\mathbf{i}\left(h_{s}\right)}, \text { where } h_{1} \cdots h_{r} \text { is a cycle in } H, \\
V_{\mathbf{o}\left(h_{1}\right), b} \otimes V_{\mathbf{o}\left(h_{1}\right)} \otimes V_{\mathbf{i}\left(h_{1}\right)}^{\otimes 2} \otimes \cdots \otimes & V_{\mathbf{i}\left(h_{s-1}\right)}^{\otimes 2} \otimes V_{\mathbf{i}\left(h_{s}\right)} \otimes V_{\mathbf{i}\left(h_{s}\right), b^{\prime}}, \\
& \text { where } b \in \mathcal{B}_{\mathbf{o}\left(h_{1}\right)}, b^{\prime} \in \mathcal{B}_{\mathbf{i}\left(h_{s}\right)}, h_{1} \cdots h_{r} \text { is a path in } H,
\end{aligned}
$$

then by applying Proposition 9.1.2 the space of $\mathrm{G}_{\mathbf{v}^{0}}^{\tau}$-invariant linear forms on $T$ is spanned by the tensor products of the $f_{x}$ in (128) for various $x$. The latters in turn are products of various functions in (126) and (127). Now following the proof of [L98, Theorem 1.3] we see that the space of $\mathrm{G}_{\mathbf{v}^{0}}^{\tau}$-invariant linear forms on $T$ is spanned by products of linear forms in (126) and (127). Theorem 9.1.1) is thus proved.

9.2. The closed immersion $\iota$. Recall $\Lambda\left(\mathbf{v}^{0}, \mathbf{w}^{0}\right)=\Lambda_{\zeta_{\mathbb{C}}}\left(\mathbf{v}^{0}, \mathbf{w}^{0}\right)$ from (6) with $\zeta_{\mathbb{C}}=0$. We can consider the categorical quotient $\Lambda\left(\mathbf{v}^{0}, \mathbf{w}^{0}\right)^{\tau} / / \mathrm{G}_{\mathbf{v}^{0}}^{\tau}$. Unlike $\mathfrak{M}_{0}\left(\mathbf{v}^{0}, \mathbf{w}^{0}\right)^{\tau}$, the variety $\Lambda\left(\mathbf{v}^{0}, \mathbf{w}^{0}\right)^{\tau} / / \mathrm{G}_{\mathbf{v}^{0}}^{\tau}$ depends on the forms associated to $\mathbf{v}^{0}$. For example, when $\Gamma=A_{1}$ and the forms on $\mathbf{v}^{0}, \mathbf{w}^{0}$ do not alternate, $\Lambda\left(\mathbf{v}^{0}, \mathbf{w}^{0}\right)^{\tau} / /$ $\mathrm{G}_{\mathbf{v}^{0}}^{\tau}=\{\mathrm{pt}\}$; otherwise it is isomorphic to the determinantal variety in $\mathfrak{g}\left(\mathbf{w}^{0}\right)$ of endomorphisms of rank $\leq \operatorname{dim} \mathbf{v}^{0}$. For the latter fact, we refer the reader to [KP82, Theorem 1.2].

By the universality of the categorical quotient, there is a morphism

$$
\Lambda\left(\mathbf{v}^{0}, \mathbf{w}^{0}\right)^{\tau} / / \mathrm{G}_{\mathbf{v}^{0}}^{\tau} \rightarrow \Lambda\left(\mathbf{v}^{0}, \mathbf{w}^{0}\right) / / \mathrm{G}_{\mathbf{v}^{0}}=\mathfrak{M}_{0}\left(\mathbf{v}^{0}, \mathbf{w}^{0}\right),
$$

which factors through $\mathfrak{M}_{0}\left(\mathbf{v}^{0}, \mathbf{w}^{0}\right)^{\tau}$ so that we have a morphism of varieties:

$$
\iota: \Lambda\left(\mathbf{v}^{0}, \mathbf{w}^{0}\right)^{\tau} / / \mathrm{G}_{\mathbf{v}^{0}}^{\tau} \rightarrow \mathfrak{M}_{0}\left(\mathbf{v}^{0}, \mathbf{w}^{0}\right)^{\tau} .
$$


Proposition 9.2.1. The morphism $\iota$ in (130) is a closed immersion for an arbitrary graph.

Proof. It is enough to show that the induced map

$$
\mathbb{C}\left[\Lambda\left(\mathbf{v}^{0}, \mathbf{w}^{0}\right)\right]^{\mathrm{G}_{\mathbf{v}^{0}}} \rightarrow \mathbb{C}\left[\Lambda\left(\mathbf{v}^{0}, \mathbf{w}^{0}\right)^{\tau}\right]^{\mathrm{G}^{\tau} \mathbf{v}^{0}}
$$

of the inclusion $\Lambda\left(\mathbf{v}^{0}, \mathbf{w}^{0}\right)^{\tau} \rightarrow \Lambda\left(\mathbf{v}^{0}, \mathbf{w}^{0}\right)$ is surjective. But this is the case by L98, Theorem 1.3] and Theorem 9.1.1. The proposition is thus proved.

For the remaining part of this section, we assume that $\Gamma$ is of type $A_{n}$. When $\mathbf{w}_{i}^{0}=0$ for all $i \geq 2$, the variety $\Lambda\left(\mathbf{v}^{0}, \mathbf{w}^{0}\right)^{\tau} / / \mathrm{G}_{\mathbf{v}^{0}}^{\tau}$ is studied by Kraft-Procesi in KP82. The generalization to arbitrary $\mathbf{w}^{0}$ is mentioned by Nakajima implicitly in [N94, Remark 8.5.4] and explicitly in [N15, Appendix A(ii)]. See also [K90]. Now we shall sharpen the previous result in type $A_{n}$. By Proposition 8.1.2, we have a closed immersion:

$$
\Lambda\left(\mathbf{v}^{0}, \mathbf{w}^{0}\right)^{\tau} \stackrel{\Phi}{\longrightarrow} \Lambda\left(\widetilde{\mathbf{v}}^{0}, \widetilde{\mathbf{w}}^{0}\right)^{\widetilde{\tau}}
$$

There is a natural imbedding $G_{\mathbf{v}^{0}} \rightarrow G_{\widetilde{\mathbf{v}}^{0}}$ with respect to the decomposition (88), which restricts to an imbedding $\mathrm{G}_{\mathbf{v}^{0}}^{\tau} \rightarrow \mathrm{G}_{\widetilde{\mathbf{v}}^{0}}^{\widetilde{\tau}}$. This induces a morphism of varieties

$$
\Lambda\left(\mathbf{v}^{0}, \mathbf{w}^{0}\right)^{\tau} / / \mathrm{G}_{\mathbf{v}^{0}}^{\tau} \stackrel{\varphi_{0}^{\prime}}{\longrightarrow} \Lambda\left(\widetilde{\mathbf{v}}^{0}, \widetilde{\mathbf{w}}^{0}\right)^{\widetilde{\tau}} / / \mathrm{G}_{\widetilde{\mathbf{v}}^{0}}^{\widetilde{\tau}} .
$$

Putting (130) and (131) together yields the following commutative diagram:

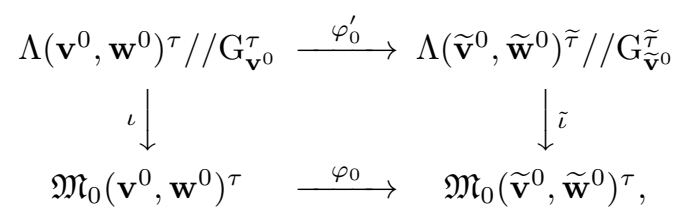

where $\iota$ and $\tilde{\iota}$ are the morphisms defined in (130).

Proposition 9.2.2. When the graph is of Dynkin type $\mathrm{A}_{n}$ and the signs $\tilde{\delta}_{\mathbf{v}^{0}}, \delta_{\mathbf{w}^{0}}$ alternate, the morphism $\varphi_{0}^{\prime}$ in (131) is a closed immersion and $\tilde{\iota}$ in (132) is an isomorphism.

Proof. We have a commutative diagram

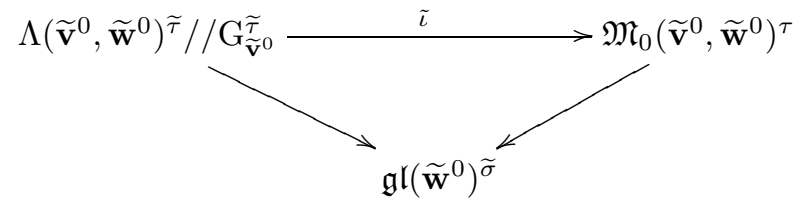

where the morphism on the right is from (120) and the one on the left is defined in a similar way. Both morphisms are closed immersions with the same image by Theorem 8.3.3 and (a slightly general version of) [KP82, Theorem 5.3], which implies that $\tilde{\iota}$ is isomorphic.

Since $\varphi_{0}$ and $\iota$ are closed immersions, so is $\varphi_{0}^{\prime}$ by using the commutative diagram (132). The proposition is thus proved.

Remark 9.2.3. In light of K90, N15 and Theorem 8.3.3, it is expected that $\iota$ in (130) is an isomorphism for a Dynkin graph of type $\mathrm{A}_{n}$. We conjecture that this holds for any graph. 
Most results in this section can be extended to a more general situation where the isomorphism $a$ in Section 3.3 is involved in a straightforward manner. We end this section with a remark on the connection with [N15, (Ai), (Aiii), (Aiv)], which is grown out from a discussion with Professor H. Nakajima.

Remark 9.2.4.

(1) Consider the Dynkin diagram of type $\mathrm{A}_{2 n+1}$. Then there is a closed-immersion similar to (130):

$$
\iota^{\prime}: \Lambda\left(\mathbf{v}^{0}, \mathbf{w}^{0}\right)^{a \tau} / / \mathrm{G}_{\mathbf{v}^{0}}^{a \tau} \rightarrow \mathfrak{M}_{0}\left(\mathbf{v}^{0}, \mathbf{w}^{0}\right)^{a \tau} .
$$

The domain of $\iota^{\prime}$ is an $S^{1}$-equivariant instanton moduli space on $\mathbb{R}^{4}$ in $[\mathrm{N15}$, (Aiii)]. Specifically, if the form on $\mathbf{w}_{i}^{0}$ is an orthogonal form for all $i$, then the domain of $\iota^{\prime}$ is exactly the $\mathrm{SO}(r)$-instantons in loc. cit., Figure 7. (Note that $\mathbf{w}_{n}^{0}$ corresponds to $w_{0}$ in Figure 7 in loc. cit.) The orthogonal/symplectic forms in loc. cit. are defined over $V_{i} \oplus V_{-i}$ and $W_{i} \oplus W_{-i}$, similar to [E09]. In our setting, we assign to $V_{n}$ a symplectic form and each $W_{i}$ and $V_{i}$ for $i \neq n$ an orthogonal form $(-\mid-)$, set $W_{i}=W_{2 n-i}, V_{i}=V_{2 n-i}$. From these data, we can obtain orthogonal/symplectic forms used in loc. cit. on $W_{i} \oplus W_{-i}$ or $V_{i} \oplus V_{2 n-i}$ by the rule $\left[\left(u_{1}, u_{2}\right),\left(w_{1}, w_{2}\right)\right]=$ $\left(u_{1} \mid w_{2}\right) \pm\left(u_{2} \mid w_{1}\right)$, where the choice of + leads to an orthogonal form and the choice of - leads to a symplectic form as desired. Under this setting, the domain of $\iota^{\prime}$ is exactly the instanton moduli space given in Figure 7 of loc. cit. Note that in this setting, the orders of $a$ and $\tau$ are 4, while their composition $a \tau$ has order 2 .

(2) If our graph allows loops, the arguments in this section still work through, with a minor modification in the proof of Theorem 9.1.1. In particular, when the graph is a Jordan quiver, i.e., a vertex with two arrows, then we have a closedimmersion

$$
\iota^{\prime \prime}: \Lambda\left(\mathbf{v}^{0}, \mathbf{w}^{0}\right)^{-a \tau} / / \mathrm{G}_{\mathbf{v}^{0}}^{a \tau} \rightarrow \mathfrak{M}_{0}\left(\mathbf{v}^{0}, \mathbf{w}^{0}\right)^{a \tau},
$$

where $a$ is induced by the obvious involution on the Jordan quiver. The domain of $\iota^{\prime \prime}$ is an SO/Sp instanton moduli space on $\mathbb{R}^{4}$ in [N15, (Ai)]. (See [Ch16] for further details.)

(3) Let $H$ be a finite subgroup in $\mathrm{SU}(2)$. By taking the $H$-equivariant parts in $\iota^{\prime \prime}$, one obtains a similar closed immersion whose domain is exactly the SO/Sp instanton moduli space on $\mathbb{R}^{4} / H$, which is discussed in [N15, (Aiv)].

Composing $a \tau$ or $\tau$ with the reflection functor $S_{w_{0}}$, it also gives rise to the $\mathrm{SO} / \mathrm{Sp}$ instanton moduli space on ALE spaces if $\mathfrak{M}_{0}\left(\mathbf{v}^{0}, \mathbf{w}^{0}\right)$ is replaced by $\mathfrak{M}_{\zeta}^{\mathrm{reg}}\left(\mathbf{v}^{0}, \mathbf{w}^{0}\right)$ for $\zeta$ generic. In particular, if the McKay diagram of $\mathrm{H}$ is of type $\mathrm{D}_{2 n}^{(1)}, \mathrm{E}_{7}^{(1)}$, or $\mathrm{E}_{8}^{(1)}$, one uses $\tau S_{w_{0}}$, and $a \tau S_{w_{0}}$ is used for the remaining cases $\mathrm{A}_{n}^{(1)}, \mathrm{D}_{2 n+1}^{(1)}$, and $\mathrm{E}_{6}^{(1)}$. This is known to Nakajima (see [N18) and is implicitly given in [N03, Sect. 9].

\section{QUiver VARIETIES AND SYMMETRIC SPACES}

In this section, we study fixed-point subvarieties of Nakajima varieties under an antisymplectic automorphism. In the type A case, we identify them with the symmetric space of a given symmetric pair of type AI/AII.

10.1. The antisymplectic automorphism $\hat{\tau}_{\zeta}$. Similar to $\tau_{\zeta}$, we define a simpler automorphism

$$
\hat{\tau}: \mathbf{M}(\mathbf{v}, \mathbf{w}) \rightarrow \mathbf{M}(\mathbf{v}, \mathbf{w}), \mathbf{x}=\left(x_{h}, p_{i}, q_{i}\right) \mapsto\left({ }^{\hat{\tau}} x_{h},{ }^{\hat{\tau}} p_{i},{ }^{\hat{\tau}} q_{i}\right),
$$


where ${ }^{\hat{\tau}} x_{h}=x_{\bar{h}}^{*},{ }^{\hat{\tau}} p_{i}=q_{i}^{*},{ }^{\hat{\tau}} q_{i}=p_{i}^{*} \quad \forall h \in H, i \in I$. The $\hat{\tau}_{\zeta}$ only differs from $\tau_{\zeta}$ by a minus sign at $x_{h}$ for $h \in \varepsilon^{-1}(-1)$ and $p_{i}$. Despite this minor perturbation, the new automorphism behaves quite differently from $\tau_{\zeta}$, as we shall see in the following, and yet proofs are always in parallel with the old ones with minor modifications, which often involve the removal of minus signs. It is easy to see that $\mu\left({ }^{\hat{T}} \mathbf{x}\right)=\mu(\mathbf{x})^{*}$. So it induces an isomorphism on $\mathfrak{M}_{\zeta}(\mathbf{v}, \mathbf{w})$ :

$$
\hat{\tau}_{\zeta}: \mathfrak{M}_{\left(\xi, \zeta_{\mathbb{C}}\right)}(\mathbf{v}, \mathbf{w}) \rightarrow \mathfrak{M}_{\left(-\xi, \zeta_{\mathbb{C}}\right)}(\mathbf{v}, \mathbf{w}) .
$$

It is also clear that $\hat{\tau}_{\zeta}$ is independent of the choices of forms on $V$ by the same argument for the similar property of $\tau_{\zeta}$. In contrast with its symplectic analogue $\tau_{\zeta}$, the $\hat{\tau}_{\zeta}$ is antisymplectic, that is,

$$
\omega\left({ }^{\hat{\tau}} \mathbf{x},{ }^{\hat{\tau}} \mathbf{x}^{\prime}\right)=-\omega\left(\mathbf{x}, \mathbf{x}^{\prime}\right),
$$

which can be verified by definition. Now we determine the order of $\hat{\tau}_{\zeta}$.

Proposition 10.1.1. If the forms on $W$ are uniform, i.e., $\delta_{\mathbf{w}, i}=\delta_{\mathbf{w}, j}$ for all $i, j \in I$, then the $\hat{\tau}_{\zeta}$ is involutive: $\hat{\tau}_{\zeta}^{2}=1$. In general, if $W$ is a formed space with sign $\delta_{\mathbf{w}}$, then $\hat{\tau}_{\zeta}^{4}=1$.

Proof. The proof follows the same line as that of Proposition 3.2.2 with the observation that $\hat{\tau}^{2}([\mathbf{x}])=\left[\left(x_{h}, \delta_{\mathbf{w}, i} p_{i}, \delta_{\mathbf{w}, i} q_{i}\right)\right]=[\mathbf{x}]$, where the last equality is given by the action of the element $\left(\delta_{\mathbf{w}, i} \operatorname{id}_{V_{i}}\right)_{i \in I} \in \mathrm{G}_{\mathbf{v}}$. The above observation indicates that $\hat{\tau}_{\zeta}^{4}=1$. The proposition is thus proved.

It is clear that the isomorphism $\hat{\tau}_{\zeta}$ commutes with the isomorphisms $a$ and $S_{\omega}$.

Lemma 10.1.2. One has $S_{i} \hat{\tau}_{\zeta}=\hat{\tau}_{s_{i} \zeta} S_{i}$ and $a \hat{\tau}_{\zeta}=\hat{\tau}_{a \zeta} a$.

10.2. The $\hat{\sigma}$-quiver varieties. Similar to $\sigma$, we consider the following isomorphism:

$$
\hat{\sigma}:=a S_{\omega} \hat{\tau}_{\zeta}: \mathfrak{M}_{\left(\xi, \zeta_{\mathbb{C}}\right)}(\mathbf{v}, \mathbf{w}) \rightarrow \mathfrak{M}_{\left(-a \omega \xi, a \omega \zeta_{\mathbb{C}}\right)}(a \omega * \mathbf{v}, a \mathbf{w}) \quad \forall \omega \in \mathcal{W}
$$

The $\hat{\sigma}$-quiver variety is defined to be

$$
\mathfrak{P}_{\zeta}(\mathbf{v}, \mathbf{w}) \equiv \mathfrak{M}_{\zeta}(\mathbf{v}, \mathbf{w})^{\hat{\sigma}},
$$

whenever $a \omega \zeta_{\mathbb{C}}=\zeta_{\mathbb{C}},-a \omega \xi=\xi$ and $a \omega * \mathbf{v}=\mathbf{v}$. By summing over all $\mathbf{v}$, we have

$$
\mathfrak{P}_{\zeta}(\mathbf{w}) \equiv \mathfrak{M}_{\zeta}(\mathbf{w})^{\hat{\sigma}} .
$$

It is clear that $\mathfrak{P}_{\zeta}(\mathbf{v}, \mathbf{w})$, and hence $\mathfrak{P}_{\zeta}(\mathbf{w})$, is independent of the choice of the form on $V$, due to the same property on $\tau_{\zeta}$. Since the $a$ and $S_{\omega}$ are symplectomorphisms and $\hat{\tau}_{\zeta}$ is antisymplectic, the $\hat{\sigma}$ is antisymplectic. Summing up, we have the following proposition.

Proposition 10.2.1. $\mathfrak{P}_{\zeta}(\mathbf{v}, \mathbf{w})$ is a fixed-point subvariety of $\mathfrak{M}_{\zeta}(\mathbf{v}, \mathbf{w})$ under an antisymplectic automorphism. Its definition is independent of the choice of the form on $V$. If $\zeta$ is generic, then $\mathfrak{P}_{\zeta}(\mathbf{v}, \mathbf{w})$ is smooth if it is nonempty and $\hat{\sigma}$ is of finite order. If $W$ is a formed space with sign $\delta_{\mathbf{w}}$ and $\omega$ is of finite order, then the order of $\hat{\sigma}$ is a divisor of l.c.m. $\{4,|\omega|,|a|\}$. If further $\delta_{\mathbf{w}}$ is uniform and $a^{2}=\omega^{2}=1$, then $\hat{\sigma}^{2}=1$.

Just like $\sigma$-quiver varieties, the $\hat{\sigma}$-quiver varieties include original quiver varieties. By a general property of antisymplectic involution, we have the following proposition. 
Proposition 10.2.2. If $|\hat{\sigma}|=2$, i.e., $\hat{\sigma}$ is anti-involutive, then $\mathfrak{P}_{\zeta}(\mathbf{v}, \mathbf{w})$ is a Lagrangian subvariety of $\mathfrak{M}_{\zeta}(\mathbf{v}, \mathbf{w})$. In particular, the dimension of $\mathfrak{P}_{\zeta}(\mathbf{v}, \mathbf{w})$, if nonempty, is half of the dimension of $\mathfrak{M}_{\zeta}(\mathbf{v}, \mathbf{w})$.

Via restriction, there is a proper map

$$
\pi^{\hat{\sigma}}: \mathfrak{P}_{\zeta}(\mathbf{v}, \mathbf{w}) \rightarrow \mathfrak{P}_{1}(\mathbf{v}, \mathbf{w}),
$$

where $\mathfrak{P}_{1}(\mathbf{v}, \mathbf{w})$ is defined in the same way as $\mathfrak{S}_{1}(\mathbf{v}, \mathbf{w})$ in (4.2.8).

Arguing in a similar way as the $\sigma$ case, it yields the following proposition.

Proposition 10.2.3. The map $\pi^{\hat{\sigma}}$ is $\mathrm{G}_{\mathbf{w}}^{\sigma}$-equivariant and if $\zeta_{\mathbb{C}}=0$, it is $\mathrm{G}_{\mathbf{w}}^{\sigma} \times \mathbb{C}^{\times}$equivariant.

Recall the fixed-point subgroup $\mathcal{W}^{\omega, a}$. For any $x \in \mathcal{W}^{\omega, a}$, the original reflection functor induces an action on the $\hat{\sigma}$-quiver varieties

$$
S_{x}^{\hat{\sigma}}: \mathfrak{P}_{\zeta}(\mathbf{v}, \mathbf{w}) \rightarrow \mathfrak{P}_{x \zeta}(x * \mathbf{v}, \mathbf{w}) .
$$

Further, the group $\mathcal{W}^{\omega, a}$ acts on the cohomology group $\mathrm{H}^{*}\left(\mathfrak{P}_{\zeta}(\mathbf{v}, \mathbf{w}), \mathbb{Z}\right)$ when $\mathbf{w}-$ $\mathbf{C v}=0$.

10.3. $\hat{\sigma}$-quiver varieties of type A. Recall the setting from Section 6.1. We define

$$
\mathfrak{p} \equiv \mathfrak{p}(W)=\left\{x \in \operatorname{End}(W) \mid x=x^{*}\right\} .
$$

This is called a symmetric space with respect to the symmetric pair $(\mathfrak{g l}(W), \mathfrak{g}(W))$. Let $\mathcal{N}(\mathfrak{p})$ be the variety of nilpotent elements in $\mathfrak{p}$.

Consider the automorphism $\hat{\sigma}_{1}$ on $T^{*} \mathcal{F}_{\mathbf{v}, \mathbf{w}}$ defined by $(x, F) \mapsto\left(x^{*}, F^{\perp}\right)$, and the fixed point subvariety $\left(T^{*} \mathcal{F}_{\mathbf{v}, \mathbf{w}}\right)^{\hat{\sigma}_{1}}$. Let

$$
\hat{\Pi}:\left(T^{*} \mathcal{F}_{\mathbf{v}, \mathbf{w}}\right)^{\hat{\sigma}_{1}} \rightarrow \mathcal{N}(\mathfrak{p})
$$

be the first projection.

Retain the setting from Section 6.2 In particular, $\mathbf{w}_{i}=0$ for all $i \geq 2$.

Proposition 10.3.1. Let $a=1$, and let $\omega=w_{0}$. Then the $\hat{\sigma}$ gets identified with the automorphism $\hat{\sigma}_{1}$. If further $w_{0} * \mathbf{v}=\mathbf{v}$, then there is a commutative diagram

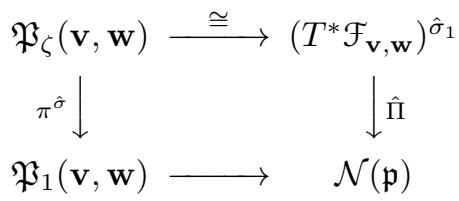

Proof. The proof is the same as that of Theorem 6.2.1 with minus signs removed.

Remark 10.3.2. The above identification implies that $\pi^{\hat{\sigma}}$ is not semismall in general. For example, when the form on $W$ is symplectic and $(\mathbf{v}, \mathbf{w})=(1,2)$, the map $\hat{\Pi}$ is the projection from the projective line $\mathbb{P}^{1}$ to a point.

Now we discuss the $\hat{\sigma}$ counterpart of the results in Section 8, Recall that it is assumed that forms on $V$ and $W$ are $\delta$-forms. We define a nondegenerate bilinear 
form $\{-\mid-\}$ on $\widetilde{V}_{i}$ by

$$
\left\{\left(v_{i}, w_{j}^{(h)}\right)_{j \geq i+h} \mid\left(v_{i}^{\prime}, u_{j}^{(h)}\right)_{j \geq i+h}\right\}_{\widetilde{V}_{i}}=\left(v_{i}, v_{i}^{\prime}\right)_{V_{i}}+\sum_{j \geq i+h}\left(w_{j}^{(h)}, u_{j}^{(j-i+1-h)}\right)_{W_{j}},
$$

where $v, v^{\prime} \in V_{i}$ and $w_{j}^{(h)}, u_{j}^{(h)} \in W_{j}^{(h)}$ such that $1 \leq h \leq j-i$.

Lemma 10.3.3. If the forms on $W$ are uniform $\delta$-forms, then so is the form on $\widetilde{W}_{1}$. If further $V$ and $W$ are uniform $\delta$-forms, then so is the form on $\widetilde{V}_{i}$, similar to $\tilde{\tau}$ in Proposition 8.1.2.

With the form $\{-\mid-\}$, one can define the automorphism $\hat{\tau}_{\{-\mid-\}}$on $\Lambda(\widetilde{V}, \widetilde{W})$.

Proposition 10.3.4. The following diagram is commutative:

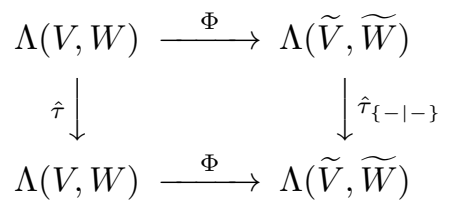

Proof. The proof is the same as that of Proposition 8.1.2 with minus signs removed at appropriate places.

Following the line of arguments in Section 8, we reach the identification of $\hat{\sigma}$ quiver varieties with nilpotent Slodowy slices in symmetric space $\mathfrak{p}$, a counterpart of Corollary 8.3.2. Let $\hat{\sigma}_{\{-\mid-\}}$denote the automorphism on $T^{*} \mathcal{F}_{\widetilde{\mathbf{v}}, \widetilde{\mathbf{w}}}$ defined with respect to the form $\{-\mid-\}$.

Theorem 10.3.5. Assume that $\zeta=(\xi, 0)$ with $\xi_{i}>0$ for all $i \in I$ and $\theta \zeta=\zeta$. Assume also that $w_{0} * \mathbf{v}=\mathbf{v}$. Then there is a commutative diagram

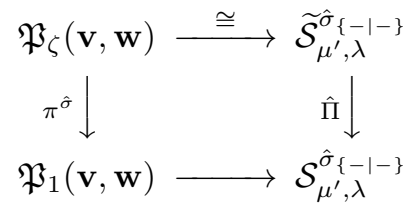

Remark 10.3.6.

(1) One still has a counterpart of Theorem 8.3.3, with $\delta_{\mathbf{w}}$ alternates replaced by $\delta_{\mathbf{w}}$ being uniform.

(2) One still has a rectangular symmetry similar to Theorem 8.4.1. Note that the $\hat{\sigma}$ therein is not the same as the $\hat{\sigma}$ in this section.

(3) One still has the column/row removal reduction in the symmetric space setting, similar to Propositions 8.5.1 8.5.2. This is a refinement of results in O86] (see also 091).

(4) Via the Kostant-Sekiguchi-Vergne correpondence [S87, Ve95] and the works of Barbasch-Sepanski [BS98, Theorem 2.3] and Chen-Nadler [CN18, we see that results in the preceding remarks can be transported, at least diffeomorphically, onto the nilpotent Slowdowy slices of the associated real groups.

Finally, we return to study the relationship between fixed-point subvarieties and categorical quotients. We assume that forms on $V$ and $W$ are uniform. We can consider the fixed-point subvariety $\mathbf{M}(\mathbf{v}, \mathbf{w})^{\hat{\tau}}$ under $\hat{\tau}$. We can define the $\mathrm{G}_{\mathbf{v}^{-}}^{\tau}$ invariant functions $\operatorname{tr}_{h_{1}, \ldots, h_{s}}(-)$ and $\chi_{h_{1}, \ldots, h_{s}}(-)$ in exactly the same manner as 
(111) and (112). Then using a similar argument as the proof of Theorem 9.1.1, we have the follwoing proposition.

Proposition 10.3.7. The algebra of $\mathrm{G}_{\mathbf{v}}^{\tau}$-invariant regular functions on $\mathbf{M}(\mathbf{v}, \mathbf{w})^{\hat{\tau}}$ is generated by the functions $\operatorname{tr}_{h_{1}, \ldots, h_{s}}(-)$ and $\chi_{h_{1}, \ldots, h_{s}}(-)$ for various paths $h_{1}, \ldots$, $h_{s}$.

From the above proposition, we have the following.

Proposition 10.3.8. There is a closed immersion $\hat{\iota}: \Lambda(\mathbf{v}, \mathbf{w})^{\hat{\tau}} / / G_{\mathbf{v}}^{\tau} \rightarrow \mathfrak{P}_{1}(\mathbf{v}, \mathbf{w})$ with $a=1$.

\section{ACKNOWLEDGMENTS}

We thank Weiqiang Wang for fruitful collaborations, especially the work BKLW], and several enlightening conversations. We also thank Dave Hemmer, Jiuzu Hong, Jim Humphreys, Ivan Losev, George Lusztig, Hiraku Nakajima, and Catharina Stroppel for stimulating discussions.

The results in this paper were announced at the ICRT VII in Xiamen, China, July 2016, the AMS special session on "Geometric methods in representation theory," Charleston, NC, March 2017, the Taipei workshop on Lie superalgebras and related topics, National Center for Theoretical Sciences, Taipei; Colloquia in Shanghai Jiaotong University and Xiamen University, July 2017, and the Algebra Seminar at University of Virginia, November 2017. It is a pleasure to thank the organizers for the invitation.

We thank the anonymous referees for careful readings, helpful comments, and insightful suggestions.

\section{REFERENCES}

[BaK16] M. Balagovic and S. Kolb, Universal K-matrix for quantum symmetric pairs, Journal für die reine und angewandte Mathematik (2016).

[BSWW] H. Bao, P. Shan, W. Wang, and B. Webster, Categorification of quantum symmetric pairs I, Quantum Topol. 9 (2018), no. 4, 643-714, DOI 10.4171/QT/117. MR 3874000

[BW13] H. Bao and W. Wang, A new approach to Kazhdan-Lusztig theory of type B via quantum symmetric pairs (English, with English and French summaries), Astérisque 402 (2018), vii+134. MR3864017

[BW16] H. Bao and W. Wang, Canonical bases arising from quantum symmetric pairs, Invent. Math. 213 (2018), no. 3, 1099-1177, DOI 10.1007/s00222-018-0801-5. MR3842062

[BKLW] H. Bao, J. Kujawa, Y. Li, and W. Wang, Geometric Schur duality of classical type, Transform. Groups 23 (2018), no. 2, 329-389, DOI 10.1007/s00031-017-9447-4. MR3805209

[BS98] D. Barbasch and M. R. Sepanski, Closure ordering and the Kostant-Sekiguchi correspondence, Proc. Amer. Math. Soc. 126 (1998), no. 1, 311-317, DOI 10.1090/S00029939-98-04090-8. MR 1422847

[BBD82] A. A. Be1̌linson, J. Bernstein, and P. Deligne, Faisceaux pervers (French), Analysis and topology on singular spaces, I (Luminy, 1981), Astérisque, vol. 100, Soc. Math. France, Paris, 1982, pp. 5-171. MR751966

[BLM] A. A. Beilinson, G. Lusztig, and R. MacPherson, A geometric setting for the quantum deformation of $\mathrm{GL}_{n}$, Duke Math. J. 61 (1990), no. 2, 655-677, DOI 10.1215/S00127094-90-06124-1. MR.1074310

[BGP] I. N. Bernštel̆n, I. M. Gel'fand, and V. A. Ponomarev, Coxeter functors, and Gabriel's theorem (Russian), Uspehi Mat. Nauk 28 (1973), no. 2(170), 19-33. MR0393065

[B03] T. Braden, Hyperbolic localization of intersection cohomology, Transform. Groups 8 (2003), no. 3, 209-216, DOI 10.1007/s00031-003-0606-4. MR.1996415 
[BG99] A. Braverman and D. Gaitsgory, On Ginzburg's Lagrangian construction of representations of $\operatorname{GL}(n)$, Math. Res. Lett. 6 (1999), no. 2, 195-201, DOI 10.4310/MRL.1999.v6.n2.a7. MR1689209

[CN18] T.-H. Chen and D. Nadler, Kostant-Sekiguchi homeomorphisms, arXiv:1805.06564.

[Ch84] I. V. Cherednik, Factorizing particles on a half line, and root systems (Russian, with English summary), Teoret. Mat. Fiz. 61 (1984), no. 1, 35-44. MR774205

[Ch16] J. Choy, Moduli spaces of framed symplectic and orthogonal bundles on $\mathbb{P}^{2}$ and the K-theoretic Nekrasov partition functions, J. Geom. Phys. 106 (2016), 284-304, DOI 10.1016/j.geomphys.2016.04.011. MR.3508922

[BOR15] E. Briand, R. Orellana, and M. Rosas, Rectangular symmetries for coefficients of symmetric functions, Electron. J. Combin. 22 (2015), no. 3, Paper 3.15, 18. MR3386516

[CG] N. Chriss and V. Ginzburg, Representation theory and complex geometry, Birkhäuser Boston, Inc., Boston, MA, 1997. MR1433132

[D] J. Dixmier, Enveloping algebras, Graduate Studies in Mathematics, vol. 11, American Mathematical Society, Providence, RI, 1996. Revised reprint of the 1977 translation. MR.1393197

[DG14] V. Drinfeld and D. Gaitsgory, On a theorem of Braden, Transform. Groups 19 (2014), no. 2, 313-358, DOI 10.1007/s00031-014-9267-8. MR3200429

[E92] B. Edixhoven, Néron models and tame ramification, Compositio Math. 81 (1992), no. 3, 291-306. MR 1149171

[ES12] M. Ehrig and C. Stroppel, 2-row Springer fibres and Khovanov diagram algebras for type D, Canad. J. Math. 68 (2016), no. 6, 1285-1333, DOI 10.4153/CJM-2015-051-4. MR3563723

[ES13] M. Ehrig and C. Stroppel, Nazarov-Wenzl algebras, coideal subalgebras and categorified skew Howe duality, Adv. Math. 331 (2018), 58-142, DOI 10.1016/j.aim.2018.01.013. MR 3804673

[E09] N. Enomoto, A quiver construction of symmetric crystals, Int. Math. Res. Not. IMRN 12 (2009), 2200-2247, DOI 10.1093/imrn/rnp014. MR2511909

[FRT] N. Yu. Reshetikhin, L. A. Takhtadzhyan, and L. D. Faddeev, Quantization of Lie groups and Lie algebras (Russian), Algebra i Analiz 1 (1989), no. 1, 178-206; English transl., Leningrad Math. J. 1 (1990), no. 1, 193-225. MR1015339

[Fu03] B. Fu, Symplectic resolutions for nilpotent orbits, Invent. Math. 151 (2003), no. 1, 167-186, DOI 10.1007/s00222-002-0260-9. MR 1943745

[G91] V. Ginzburg, Lagrangian construction of the enveloping algebra $U\left(\mathrm{sl}_{n}\right)$ (English, with French summary), C. R. Acad. Sci. Paris Sér. I Math. 312 (1991), no. 12, 907-912. MR.1111326

[GRWa] N. Guay, V. Regelskis, and C. Wendlandt, Representations of twisted Yangians of types $B, C$, D: I, Selecta Math. (N.S.) 23 (2017), no. 3, 2071-2156, DOI 10.1007/s00029-0170306-x. MR 3663603

[GRWb] N. Guay, V. Regelskis, and C. Wendlandt, Representations of twisted Yangians of types $B, C, D: I I$, arXiv:1708.00968.

[H] S. Helgason, Differential geometry, Lie groups, and symmetric spaces, Pure and Applied Mathematics, vol. 80, Academic Press, Inc. [Harcourt Brace Jovanovich, Publishers], New York-London, 1978. MR514561

[HL14] A. Henderson and A. Licata, Diagram automorphisms of quiver varieties, Adv. Math. 267 (2014), 225-276, DOI 10.1016/j.aim.2014.08.007. MR.3269179

[H15] A. Henderson, Singularities of nilpotent orbit closures, Rev. Roumaine Math. Pures Appl. 60 (2015), no. 4, 441-469. MR3436211

[I72] B. Iversen, A fixed point formula for action of tori on algebraic varieties, Invent. Math. 16 (1972), 229-236, DOI 10.1007/BF01425495. MR0299608

[K14] S. Kolb, Quantum symmetric Kac-Moody pairs, Adv. Math. 267 (2014), 395-469, DOI 10.1016/j.aim.2014.08.010. MR.3269184

[KR71] B. Kostant and S. Rallis, Orbits and representations associated with symmetric spaces, Amer. J. Math. 93 (1971), 753-809, DOI 10.2307/2373470. MR0311837

[KP81] H. Kraft and C. Procesi, Minimal singularities in $\mathrm{GL}_{n}$, Invent. Math. 62 (1981), no. 3, 503-515, DOI 10.1007/BF01394257. MR604841

[KP82] H. Kraft and C. Procesi, On the geometry of conjugacy classes in classical groups, Comment. Math. Helv. 57 (1982), no. 4, 539-602, DOI 10.1007/BF02565876. MR694606 
[K90] P. B. Kronheimer, Instantons and the geometry of the nilpotent variety, J. Differential Geom. 32 (1990), no. 2, 473-490. MR1072915

[KN90] P. B. Kronheimer and H. Nakajima, Yang-Mills instantons on ALE gravitational instantons, Math. Ann. 288 (1990), no. 2, 263-307, DOI 10.1007/BF01444534. MR1075769

[Le] G. Letzter, Harish-Chandra modules for quantum symmetric pairs, Represent. Theory 4 (2000), 64-96, DOI 10.1090/S1088-4165-00-00087-X. MR.1742961

[LW15] Y. Li and W. Wang, Positivity vs negativity of canonical bases, Bull. Inst. Math. Acad. Sin. (N.S.) 13 (2018), no. 2, 143-198. MR3792711

[L98] G. Lusztig, On quiver varieties, Adv. Math. 136 (1998), no. 1, 141-182, DOI 10.1006/aima.1998.1729. MR 1623674

[L00] G. Lusztig, Quiver varieties and Weyl group actions (English, with English and French summaries), Ann. Inst. Fourier (Grenoble) 50 (2000), no. 2, 461-489. MR1775358

[L00b] G. Lusztig, Remarks on quiver varieties, Duke Math. J. 105 (2000), no. 2, 239-265, DOI 10.1215/S0012-7094-00-10523-6. MR1793612

[M02] A. Maffei, A remark on quiver varieties and Weyl groups, Ann. Sc. Norm. Super. Pisa Cl. Sci. (5) 1 (2002), no. 3, 649-686. MR1990675

[M05] A. Maffei, Quiver varieties of type A, Comment. Math. Helv. 80 (2005), no. 1, 1-27, DOI $10.4171 / \mathrm{CMH} / 1$. MR2130242

[MO12] D. Maulik and A. Okounkov, Quantum groups and quantum cohomology, arXiv:1211.1287.

[M07] A. Molev, Yangians and classical Lie algebras, Mathematical Surveys and Monographs, vol. 143, American Mathematical Society, Providence, RI, 2007. MR2355506

[N94] H. Nakajima, Instantons on ALE spaces, quiver varieties, and Kac-Moody algebras, Duke Math. J. 76 (1994), no. 2, 365-416, DOI 10.1215/S0012-7094-94-07613-8. MR.1302318

[N96] H. Nakajima, Varieties associated with quivers, Representation theory of algebras and related topics (Mexico City, 1994), CMS Conf. Proc., vol. 19, Amer. Math. Soc., Providence, RI, 1996, pp. 139-157. MR1388562

[N98] H. Nakajima, Quiver varieties and Kac-Moody algebras, Duke Math. J. 91 (1998), no. 3, 515-560, DOI 10.1215/S0012-7094-98-09120-7. MR1604167

[N00] H. Nakajima, Quiver varieties and finite-dimensional representations of quantum affine algebras, J. Amer. Math. Soc. 14 (2001), no. 1, 145-238, DOI 10.1090/S0894-0347-0000353-2. MR 1808477

[N01] H. Nakajima, Quiver varieties and tensor products, Invent. Math. 146 (2001), no. 2, 399-449, DOI 10.1007/PL00005810. MR 1865400

[N03] H. Nakajima, Reflection functors for quiver varieties and Weyl group actions, Math. Ann. 327 (2003), no. 4, 671-721, DOI 10.1007/s00208-003-0467-0. MR2023313

[N13] H. Nakajima, Quiver varieties and tensor products, II, Symmetries, integrable systems and representations, Springer Proc. Math. Stat., vol. 40, Springer, Heidelberg, 2013, pp. 403-428, DOI 10.1007/978-1-4471-4863-0_16. MR3077693

[N15] H. Nakajima, Towards a mathematical definition of Coulomb branches of 3-dimensional $\mathcal{N}=4$ gauge theories, I, Adv. Theor. Math. Phys. 20 (2016), no. 3, 595-669, DOI 10.4310/ATMP.2016.v20.n3.a4. MR.3565863

[N16] H. Nakajima, Lectures on perverse sheaves on instanton moduli spaces, Geometry of moduli spaces and representation theory, IAS/Park City Math. Ser., vol. 24, Amer. Math. Soc., Providence, RI, 2017, pp. 381-436. MR3752464

[N18] H. Nakajima, Instantons on ALE spaces, quiver varieties, and Kac-Moody algebras, Duke Math. J. 76 (1994), no. 2, 365-416, DOI 10.1215/S0012-7094-94-07613-8. MR:1302318

[O86] T. Ohta, The singularities of the closures of nilpotent orbits in certain symmetric pairs, Tohoku Math. J. (2) 38 (1986), no. 3, 441-468, DOI 10.2748/tmj/1178228456. MR 854462

[O91] T. Ohta, The closures of nilpotent orbits in the classical symmetric pairs and their singularities, Tohoku Math. J. (2) 43 (1991), no. 2, 161-211, DOI 10.2748/tmj/1178227492. MR.1104427

[OV] A. L. Onishchik and È. B. Vinberg, Lie groups and algebraic groups, Springer Series in Soviet Mathematics, Springer-Verlag, Berlin, 1990. Translated from the Russian and with a preface by D. A. Leites. MR.1064110 
[S84] J. Sekiguchi, The nilpotent subvariety of the vector space associated to a symmetric pair, Publ. Res. Inst. Math. Sci. 20 (1984), no. 1, 155-212, DOI 10.2977/prims/1195181836. MR736100

[S87] J. Sekiguchi, Remarks on real nilpotent orbits of a symmetric pair, J. Math. Soc. Japan 39 (1987), no. 1, 127-138, DOI 10.2969/jmsj/03910127. MR867991

[S180a] P. Slodowy, Four lectures on simple groups and singularities, Communications of the Mathematical Institute, Rijksuniversiteit Utrecht, vol. 11, Rijksuniversiteit Utrecht, Mathematical Institute, Utrecht, 1980. MR563725

[S180b] P. Slodowy, Simple singularities and simple algebraic groups, Lecture Notes in Mathematics, vol. 815, Springer, Berlin, 1980. MR.584445

[VV00] M. Varagnolo and E. Vasserot, Standard modules of quantum affine algebras, Duke Math. J. 111 (2002), no. 3, 509-533, DOI 10.1215/S0012-7094-02-11135-1. MR1885830

[VV03] M. Varagnolo and E. Vasserot, Canonical bases and quiver varieties, Represent. Theory 7 (2003), 227-258, DOI 10.1090/S1088-4165-03-00154-7. MR1990661

[Ve95] M. Vergne, Instantons et correspondance de Kostant-Sekiguchi (French, with English and French summaries), C. R. Acad. Sci. Paris Sér. I Math. 320 (1995), no. 8, 901-906. MR.1328708

[V86] D. A. Vogan Jr., The orbit method and primitive ideals for semisimple Lie algebras, Lie algebras and related topics (Windsor, Ont., 1984), CMS Conf. Proc., vol. 5, Amer. Math. Soc., Providence, RI, 1986, pp. 281-316. MR832204

[V89] D. A. Vogan Jr., Associated varieties and unipotent representations, Harmonic analysis on reductive groups (Brunswick, ME, 1989), Progr. Math., vol. 101, Birkhäuser Boston, Boston, MA, 1991, pp. 315-388. MR1168491

[W39] H. Weyl, The classical groups, Princeton Landmarks in Mathematics, Princeton University Press, Princeton, NJ, 1997. Their invariants and representations; Fifteenth printing; Princeton Paperbacks. MR 1488158

[W15] A. Wilbert, Topology of two-row Springer fibers for the even orthogonal and symplectic group, Trans. Amer. Math. Soc. 370 (2018), no. 4, 2707-2737, DOI 10.1090/tran/7194. MR 3748583

Department of Mathematics, University at Buffalo, the State University of New York, Buffalo, New York 14260

Email address: yiqiang@buffalo.edu 